\title{
QUIVERS, PERVERSE SHEAVES, AND QUANTIZED ENVELOPING ALGEBRAS
}

\author{
G. LUSZTIG
}

\section{Contents}

1. Preliminaries

2. A class of perverse sheaves on $\mathbf{E}_{\mathbf{V}, \Omega}$

3. Multiplication

4. Restriction

5. Fourier-Deligne transform

6. Analysis of a sink

7. Multiplicative generators

8. Compatibility of multiplication with restriction

9. Rank 2

10. Definition of the canonical basis $\mathbf{B}$ of $\mathbf{U}^{-}$

11. Properties of the canonical basis $\mathbf{B}$ of $\mathbf{U}^{-}$

12. The variety $\Lambda_{\mathbf{V}}$

13. Singular supports

14. Example: graphs of type $A, D, E$

15. Example: graphs of affine type $A$

16. Graphs with a cyclic group action

\section{INTRODUCTION}

0.1. Let us consider a finite graph in which no edge joins a vertex with itself. This graph defines a symmetric matrix $\left(a_{i j}\right)$ indexed by pairs of vertices of the graph, where $a_{i i}=2$ and $-a_{i j}$ is the number of edges joining $i, j$ if $i \neq j$. Let $\mathbf{g}$ be the Lie algebra over $\mathbf{Q}$ defined in terms of this matrix by the usual Serre relations (a Kac-Moody Lie algebra), let $u$ be its enveloping algebra, and let $\mathbf{U}$ be the quantized version of $u$ discovered by Drinfeld and Jimbo (a Hopf algebra over $\mathbf{Q}(v))$.

Let $u=u^{-} \otimes u^{0} \otimes u^{+}$(resp. $\mathbf{U}=\mathbf{U}^{-} \otimes \mathbf{U}^{0} \otimes \mathbf{U}^{+}$) be the triangular decomposition of $u$ (resp. $\mathbf{U})$.

Received by the editors January 4, 1991.

1991 Mathematics Subject Classification. Primary 20G99.

Supported in part by National Science Foundation Grant DMS 8702842. 
0.2. The main discovery of [L2] was the existence of a canonical basis for $\mathbf{U}^{-}$ (in case of graphs of type $A, D, E$ ) with some very remarkable properties. Among these properties were integrality, a positivity property for structure constants, compatibility with various natural filtrations, and the fact that this basis gave rise to canonical bases in all finite-dimensional irreducible representations.

This was done by two quite different methods, an elementary method and a geometric method.

0.3. In this paper, the geometric method of [L2] is extended to the case of arbitrary graphs; we obtain a canonical basis of $\mathbf{U}^{-}$with the same kind of properties as in the ordinary case.

Let us fix an orientation for our graph (or a quiver). From the work of Ringel [R] it is known that the algebras $u^{-}, \mathbf{U}^{-}$can be reconstructed purely in terms of the representation theory of this quiver, in the case of graphs of type $A, D, E$. (In a not yet written work, Ringel has extended this to the case of Dynkin graphs of affine type; using a variant of Ringel's method, Schofield [S] has extended Ringel's results on $u^{-}$, but not those on $\mathbf{U}^{-}$, to arbitrary finite graphs.)

It turns out that by looking more closely at the geometry of representations of the quiver one can get not only $\mathbf{U}^{-}$but also a canonical basis for it.

It is well known that $\mathbf{U}^{-}$is naturally graded: $\mathbf{U}^{-}=\bigoplus_{\nu} \mathbf{U}_{\nu}^{-}$where $\nu$ are functions on the vertices of our graph with values in $\mathbf{N}$ and $\mathbf{U}_{\nu}^{-}$are finite dimensional.

Assume that we are given a complex vector space $\mathbf{V}_{i}$ for each vertex $i$ of our graph so that $\operatorname{dim} \mathbf{V}_{i}=\nu(i)$.

Let $\mathbf{E}=\bigoplus \operatorname{Hom}\left(\mathbf{V}_{i}, \mathbf{V}_{j}\right)$ where the sum is over all arrows $i \rightarrow j$ in the orientation. This is a finite-dimensional complex vector space on which the algebraic group $G_{\mathbf{V}}=\prod_{i}$ Aut $\mathbf{V}_{i}$ acts naturally. The points of $\mathbf{E}$ may be regarded as representations of our quiver (oriented graph).

We would like to produce from $\mathbf{E}$ finitely many objects which should parametrize a basis of $\mathbf{U}_{\nu}^{-}$.

In the $A, D, E$ case this task is easily solved: one takes the set of all $G_{\mathbf{V}^{-}}$ orbits on $\mathbf{E}$. (This set is finite by Gabriel's theorem.) This does not make sense in the general case; there may be infinitely many orbits.

To get around this difficulty, we imitate the definition of character sheaves in [L1]. As in that theory we have a finite number of varieties (corresponding to various kinds of flags) that map properly to $\mathbf{E}$. The direct image of the constant sheaf under each of these maps decomposes as a direct sum of irreducible perverse sheaves (up to shift), and the perverse sheaves that appear in this way form a finite collection of objects, which satisfies our requirements.

We can show that this class of perverse sheaves is closed under a certain multiplication operation; this eventually leads to a construction of $\mathbf{U}^{-}$endowed with a canonical basis provided by the perverse sheaves as above. (That basis is in fact independent of the chosen orientation.) 
0.4 . As shown by Drinfeld and Jimbo, $\mathbf{U}^{-} \otimes \mathbf{U}^{0}$ has a natural comultiplication. We are able to recover this comultiplication in the language of perverse sheaves. (We are again imitating character sheaves, namely the restriction functor of [L2].) As a consequence, we obtain a positivity property for the canonical basis, with respect to comultiplication, which is new even in the $A, D, E$ case.

0.5 . Another aspect of this work is a study of the singular supports of the perverse sheaves connected with our canonical basis. We show that these singular supports are contained in a certain remarkable Lagrangian variety (already introduced in [L3]) whose definition is again reminiscent of what happens for character sheaves.

0.6. In a sequel to this paper, we will describe explicitly the perverse sheaves connected with our canonical basis in the case of affine Dynkin graphs, in the sense that we will describe their support in the framework of the Dlab-Ringel theory [DR], and the corresponding local systems on an open part of the support. Remarkably, the theory of character sheaves of $G L_{n}$ enters in this description.

0.7. As we already mentioned, in [L2], a second construction of the canonical basis was given; this was elementary, in the sense that no results from topology or algebraic geometry were used. Soon after [L2] became available, Kashiwara announced an elementary construction in a somewhat similar spirit of a canonical basis that made sense for general graphs.

(Kashiwara's construction is given in his very interesting preprint $O$ On crystal bases of the q-analogue of universal enveloping algebras, which I received after this paper had been submitted.)

Note that the elementary approach to canonical bases, while being elegant, cannot provide positivity results of the kind provided by the perverse sheaves approach.

\section{Preliminaries}

1.1. We assume given a finite nonempty graph; in this graph, two different vertices may be joined by several edges, but no edge may join a vertex with itself. Let $I$ be the set of vertices of our graph, and let $H$ be the set of pairs consisting of an edge together with an orientation of it.

Giving such a graph is the same as giving

(a) two finite sets $I, H$ with $I$ nonempty,

(b) a map $H \rightarrow I$ denoted $h \rightarrow h^{\prime}$,

(c) a map $H \rightarrow I$ denoted $h \rightarrow h^{\prime \prime}$ and

(d) a fixed point free involution $h \rightarrow \bar{h}$ of $H$;

these are subject to

(e) $(\bar{h})^{\prime}=h^{\prime \prime}$ and

(f) $h^{\prime} \neq h^{\prime \prime}$

for all $h \in H$.

An orientation of our graph is a choice of a subset $\Omega \subset H$ such that $\Omega \cup \bar{\Omega}=$ $H, \Omega \cap \bar{\Omega}=\varnothing$. 
1.2. Let $\mathbf{k}$ be an algebraically closed field.

Let $\mathscr{V}$ be the category of finite-dimensional $I$-graded vector spaces $\mathbf{V}=$ $\bigoplus_{i \in I} \mathbf{V}_{i}$ over $\mathbf{k}$ with morphisms being linear maps respecting the grading. We shall write $\mathbf{V} \in \mathscr{V}$ to indicate that $\mathbf{V}$ is an object of $\mathscr{V}$.

Let $\mathbf{N}^{I}$ be the set of all functions $\nu: I \rightarrow \mathbf{N}$. For each $\nu \in \mathbf{N}^{I}$ we denote by $\mathscr{V}_{\nu}$ the full subcategory of $\mathscr{V}$ whose objects are those $\mathbf{V}$ such that $\operatorname{dim} \mathbf{V}_{i}=$ $\nu(i)$ for all $i \in I$. Then each object in $\mathscr{V}$ belongs to $\mathscr{V}_{\nu}$ for a unique $\nu$, and all objects of $\mathscr{V}_{\nu}$ are isomorphic to each other.

Given $\mathbf{V} \in \mathscr{V}$, let

$$
\mathbf{E}_{\mathbf{v}}=\bigoplus_{h \in H} \operatorname{Hom}\left(\mathbf{V}_{h^{\prime}}, \mathbf{v}_{h^{\prime \prime}}\right)
$$

More generally, for any subset $H^{\prime}$ of $H$ we shall consider the subspace $\mathbf{E}_{\mathbf{V}, H^{\prime}}$ of $\mathbf{E}_{\mathbf{v}}$ consisting of all vectors $x=\left(x_{h}\right)$ such that $x_{h}=0$ whenever $h \in$ $H-H^{\prime}$.

The algebraic group $G_{\mathbf{v}}=\prod_{i} \operatorname{Aut}\left(\mathbf{V}_{i}\right)$ (naturally a subgroup of $\operatorname{Aut}(\mathbf{V})$ ) acts on $\mathbf{E}_{\mathbf{V}}$ (and on $\left.\mathbf{E}_{\mathbf{v}, H^{\prime}}\right)$ by $(g, x) \rightarrow g x=x^{\prime}$ where $x_{h}^{\prime}=g_{h^{\prime \prime}} x_{h} g_{h^{\prime}}^{-1}$ for all $h$.

1.3. Let $\mathbf{V}^{\prime}$ be an $I$-graded subspace of $\mathbf{V} \in \mathscr{V}$ and let $x \in \mathbf{E}_{\mathbf{V}}$. We say that $\mathbf{V}^{\prime}$ is $x$-stable if $x_{h}\left(\mathbf{V}_{h^{\prime}}^{\prime}\right) \subset \mathbf{V}_{h^{\prime \prime}}^{\prime}$ for all $h \in H$.

In this case, we may consider the linear maps $x_{h}^{\prime}: \mathbf{V}_{h^{\prime}}^{\prime} \rightarrow \mathbf{V}_{h^{\prime \prime}}^{\prime}, x_{h}^{\prime \prime}: \mathbf{V}_{h^{\prime}} / \mathbf{V}_{h^{\prime}}^{\prime} \rightarrow$ $\mathbf{V}_{h^{\prime \prime}} / \mathbf{V}_{h^{\prime \prime}}^{\prime}$ induced by $x_{h}$, and we obtain elements $x^{\prime}=\left(x_{h}^{\prime}\right) \in \mathbf{E}_{\mathbf{V}^{\prime}}$ and $x^{\prime \prime}=$ $\left(x_{h}^{\prime \prime}\right) \in \mathbf{E}_{\mathbf{v}^{\prime}}$. We say that $x^{\prime}, x^{\prime \prime}$ are induced by $x$.

1.4. Let $\nu \in \mathbf{N}^{I}$ and suppose that $S_{\nu}$ is the set of all pairs $(\mathbf{i}, \mathbf{a})$ where $\mathbf{i}=$ $\left(i_{1}, i_{2}, \ldots, i_{m}\right)$ is a sequence of elements of $I$ and $\mathbf{a}=\left(a_{1}, a_{2}, \ldots, a_{m}\right)$ is a sequence of integers $\geq 0$ such that $\sum_{l: i_{l}=i} a_{l}=\nu(i)$ for all $i \in I$.

Now let $\mathbf{V} \in \mathscr{V}_{\nu}$ and let $(\mathbf{i}, \mathbf{a}) \in S_{\nu}$. A flag of type $(\mathbf{i}, \mathbf{a})$ in $\mathbf{V}$ is, by definition, a sequence $\phi=\left(\mathbf{V}=\mathbf{V}^{0} \supset \mathbf{V}^{1} \supset \cdots \supset \mathbf{V}^{m}=0\right)$ of $I$-graded subspaces of $\mathbf{V}$ such that, for any $l=1,2, \ldots, m$, the graded vector space $\mathbf{V}^{l-1} / \mathbf{V}^{l}$ is zero in degrees $\neq i_{l}$ and has dimension $a_{l}$ in degree $i_{l}$.

If $x \in \mathbf{E}_{\mathbf{V}}$, we say that $\phi$ is $x$-stable if $\mathbf{V}^{l}$ is $x$-stable (see 1.3) for all $h \in H$ and all $l=0,1, \ldots, m$.

Let $\mathscr{F}_{\mathbf{i}, \mathbf{a}}$ be the variety of all flags of type $(\mathbf{i}, \mathbf{a})$ in $\mathbf{V}$.

1.5. In this subsection we assume that an orientation $\Omega$ for our graph has been chosen. Let $\widetilde{F_{i, a}}$ be the variety of all pairs $(x, \phi)$ such that $x \in \mathbf{E}_{\mathbf{V}, \Omega}$ and $\phi \in \mathscr{F}_{\mathbf{i}, \mathbf{a}}$ is $x$-stable. $G_{\mathbf{V}}$ acts on $\mathscr{F}_{\mathbf{i}, \mathbf{a}}$ by $g: \phi \rightarrow g \phi$ where $\phi=\left(\mathbf{V}=\mathbf{V}^{0} \supset\right.$ $\left.\mathbf{V}^{1} \supset \cdots \supset \mathbf{V}^{m}=0\right)$ and $g \phi=\left(\mathbf{V}=g \mathbf{V}^{0} \supset g \mathbf{V}^{1} \supset \cdots \supset g \mathbf{V}^{m}=0\right)$. Hence $G_{\mathbf{V}}$ acts on $\widetilde{F}_{\mathbf{i}, \mathbf{a}}$ by $g:(x, \phi) \rightarrow(g x, g \phi)$.

We denote by $\pi_{\mathrm{i}, \mathrm{a}}: \widetilde{\mathscr{F}}_{\mathrm{i}, \mathrm{a}} \rightarrow \mathbf{E}_{\mathrm{V}, \Omega}$ the first projection. With these notations, we have the following result. 
Lemma 1.6. (a) $\mathscr{F}_{\mathbf{i}, \mathbf{a}}$ is a smooth, irreducible, projective variety of dimension

$$
\sum_{l<l^{\prime}: i_{l^{\prime}}=i_{l}} a_{l^{\prime}} a_{l}
$$

and $G_{\mathbf{v}}$ acts transitively on it. sion

(b) The second projection $\widetilde{\mathscr{F}}_{\mathbf{i}, \mathbf{a}} \rightarrow \mathscr{F}_{\mathbf{i}, \mathbf{a}}$ is a vector bundle with fibres of dimen-

$$
\sum_{l^{\prime} \leq l} \sharp\left\{h \in \Omega \mid h^{\prime}=i_{l^{\prime}}, h^{\prime \prime}=i_{l}\right\} a_{l^{\prime}} a_{l^{\prime}}
$$

(c) $\widetilde{\mathscr{F}}_{\mathbf{i}, \mathbf{a}}$ is a smooth, irreducible variety of dimension

$$
\sum_{l^{\prime} \leq l} \sharp\left\{h \in \Omega \mid h^{\prime}=i_{l^{\prime}}, h^{\prime \prime}=i_{l}\right\} a_{l^{\prime}} a_{l}+\sum_{l<l^{\prime}: i_{l^{\prime}}=i_{l}} a_{l^{\prime}} a_{l} .
$$

(d) $\pi_{\mathbf{i}, \mathbf{a}}$ is a proper morphism.

(e) $\pi_{\mathbf{i}, \mathbf{a}}$ is $G_{\mathbf{v}}$-equivariant.

(a) holds since $\mathscr{F}_{\mathbf{i}, \mathbf{a}}$ is isomorphic to a product over $i \in I$ of usual (partial) flag manifolds attached to $\mathbf{V}_{i}$. (d) follows from (a); (e) is obvious and (c) follows from (b) and (a). It remains to prove (b). Let us fix $\phi=\left(\mathbf{V}=\mathbf{V}^{0} \supset\right.$ $\left.\mathbf{V}^{1} \supset \cdots \supset \mathbf{V}^{m}=0\right)$ in $\mathscr{F}_{\mathbf{i}, \mathbf{a}}$ and let $Z$ be the fibre at $\phi$ of the second projection in (b). The first projection identifies $Z$ with the set of all $x \in \mathbf{E}_{\mathbf{v}, \Omega}$ such that $x_{h}\left(\mathbf{V}_{h^{\prime}}^{l}\right) \subset \mathbf{V}_{h^{\prime \prime}}^{l}$ for all $h \in H$ and all $l$; this is clearly a linear subspace of $\mathbf{E}_{\mathbf{V}, \Omega}$. Its dimension is equal to

$$
\sum_{l^{\prime} \leq l ; h \in \Omega} \operatorname{dim}\left(\mathbf{V}_{h^{\prime}}^{l^{\prime}-1} / \mathbf{V}_{h^{\prime}}^{l^{\prime}}\right) \operatorname{dim}\left(\mathbf{V}_{h^{\prime \prime}}^{l-1} / \mathbf{V}_{h^{\prime \prime}}^{l}\right)
$$

hence to

$$
\sum_{l^{\prime} \leq l} \sharp\left\{h \in \Omega \mid h^{\prime}=i_{l^{\prime}}, h^{\prime \prime}=i_{l}\right\} a_{l^{\prime}} a_{l^{\prime}} .
$$

This independent of $\phi$ (this can be also seen from the transitivity of the $G_{\mathbf{V}}$-action on $\left.\mathscr{F}_{\mathbf{i}, \mathbf{a}}\right)$. The lemma follows.

1.7. An element $f \in \mathbf{E}_{\mathbf{v}}$ is said to be nilpotent if there exists an $N \geq 2$ such that the following condition is satisfied: for any sequence $h_{1}, h_{2}, \ldots, h_{N}$ in $H$ such that $h_{1}^{\prime}=h_{2}^{\prime \prime}, h_{2}^{\prime}=h_{3}^{\prime \prime}, \ldots, h_{N-1}^{\prime}=h_{N}^{\prime \prime}$, the composition $f_{h_{1}} f_{h_{2}} \cdots f_{h_{N}}$ : $\mathbf{V}_{h_{N}^{\prime}} \rightarrow \mathbf{V}_{h_{1}^{\prime \prime}}$ is zero.

Lemma 1.8. (a) If $x \in \mathbf{E}_{\mathbf{V}}$ and $\phi=\left(\mathbf{V}=\mathbf{V}^{0} \supset \mathbf{V}^{1} \supset \cdots \supset \mathbf{V}^{m}=0\right)$ is $a$ flag of type $(\mathbf{i}, \mathbf{a})$ that is $x$-stable, then $x_{h}\left(\mathbf{V}_{h^{\prime}}^{l-1}\right) \subset \mathbf{V}_{h^{\prime \prime}}^{l}$ for all $h \in H$ and $l=1,2, \ldots, m$. In particular, $x$ is nilpotent.

(b) Conversely, if $x \in \mathbf{E}_{\mathbf{v}}$ is nilpotent, then there exists (i, a) $\in S_{\nu}$ and a flag $\phi$ of type (i, a) such that $\phi$ is $x$-stable. 
Assume that we are in the setup of (a). If $h$ is such that $h^{\prime} \neq i_{l}$ then $\mathbf{V}_{h^{\prime}}^{l-1}=\mathbf{V}_{h^{\prime}}^{l}$ and hence $x_{h}\left(\mathbf{V}_{h^{\prime}}^{l-1}\right)=x_{h}\left(\mathbf{V}_{h^{\prime}}^{l}\right) \subset \mathbf{V}_{h^{\prime \prime}}^{l}$; if $h$ is such that $h^{\prime \prime} \neq i_{l}$ then $\mathbf{V}_{h^{\prime \prime}}^{l-1}=\mathbf{V}_{h^{\prime \prime}}^{l}$ and hence $x_{h}\left(\mathbf{V}_{h^{\prime}}^{l-1}\right) \subset \mathbf{V}_{h^{\prime \prime}}^{l-1}=\mathbf{V}_{h^{\prime \prime}}^{l}$.

Since for $h \in H$ we cannot have simultaneously $h^{\prime}=i_{l}$ and $h^{\prime \prime}=i_{l}$, (a) is proved.

To prove (b), we may assume that $\mathbf{V} \neq 0$ and that the result is already proved for $I$-graded vector spaces of dimension strictly smaller than that of $\mathbf{V}$.

For any $k \in I$ and any $N \geq 1$, let $Z(k, N)$ be the set of all sequences $\mathbf{h}=$ $\left(h_{1}, h_{2}, \ldots, h_{N}\right)$ in $H$ such that $h_{1}^{\prime \prime}=k, h_{i}^{\prime}=h_{i+1}^{\prime \prime}$ for $i=1,2, \ldots, N-1$.

For $\mathbf{h} \in \mathbf{Z}(k, N)$, we denote by $\mathbf{V}_{k}(\mathbf{h})$ the image of the composed map $x_{h_{1}} x_{h_{2}} \cdots x_{h_{N}}: \mathbf{V}_{h_{N}^{\prime}} \rightarrow \mathbf{V}_{k}$.

We denote $\mathbf{V}_{k}(N)=\sum_{\mathbf{h} \in Z(k, N)} \mathbf{V}_{k}(\mathbf{h})$, a subspace of $\mathbf{V}_{k}$. We also set $\mathbf{V}_{k}(0)=\mathbf{V}_{k}$.

Clearly, we have

(c) $\mathbf{V}_{k}(N)=\sum_{h \in H: h^{\prime \prime}=k} x_{h}\left(\mathbf{V}_{h^{\prime}}(N-1)\right)$ for all $N \geq 1$.

Assume that

(d) $\mathbf{V}_{k}(1)=\mathbf{V}_{k}$ for all $k \in I$.

From this we deduce by induction that

(e) $\mathbf{V}_{k}(N)=\mathbf{V}_{k}(N-1)$ for all $k \in I$ and all $N \geq 1$.

For $N=1$ this is just (d). Hence we may assume that $N \geq 2$ and $\mathbf{V}_{k}(N-1)=\mathbf{V}_{k}(N-2)$ for all $k$. Then using twice (c) we deduce that $\mathbf{V}_{k}(N)=\sum_{h \in H: h^{\prime \prime}=k} x_{h}\left(\mathbf{V}_{h^{\prime}}(N-1)\right)=\sum_{h \in H: h^{\prime \prime}=k} x_{h}\left(\mathbf{V}_{h^{\prime}}(N-2)\right)=\mathbf{V}_{k}(N-1)$ and (e) follows. From (e) we see that $\mathbf{V}_{k}=\mathbf{V}_{k}(1)=\mathbf{V}_{k}(2)=\cdots$. However, by the assumption of (b), for large $N$, we have $\mathbf{V}_{k}(N)=0$ for all $k$. It follows that $\mathbf{V}_{k}=0$. This contradiction shows that $(\mathbf{d})$ is false. Thus, there exists $k \in I$ such that $\mathbf{V}_{k}(1) \neq \mathbf{V}_{k}$.

Let $\mathbf{V}^{1}$ be the $I$-graded subspace of $\mathbf{V}$ defined by $\mathbf{V}_{k}^{1}=\mathbf{V}_{k}(1)$ and $\mathbf{V}_{i}^{1}=\mathbf{V}_{i}$ for $i \neq k$. We have $x_{h}\left(\mathbf{V}_{h^{\prime}}^{1}\right) \subset \mathbf{V}_{h^{\prime \prime}}^{1}$ for all $h \in H$. Thus the restriction of $x$ gives an element $\tilde{x} \in \mathbf{E}_{\mathbf{v}^{1}}$. It is clear that this element is nilpotent. By the induction hypothesis, we can find a flag of some type in $\mathbf{V}^{1}$ that is $\tilde{x}$-stable. This flag preceded by $\mathbf{V}$ itself constitutes a flag in $\mathbf{V}$ that is $x$-stable. Thus, (b) is proved.

\section{A ClASS OF PERVERSE SHEAVES ON $\mathbf{E}_{\mathbf{V}, \Omega}$}

2.1. In this section, as well as in $\S 3$ and $\S 4$, we assume that an orientation $\Omega$ for our graph has been chosen.

We fix a prime number $l$ invertible in $k$. We generally write $\mathscr{D}(X)$ for the bounded derived category of complexes of $\bar{Q}_{l}$-sheaves on an algebraic variety $X$ over $\mathbf{k}$. We shall use the notations of [BBD]; in particular, $[d]$ denotes a shift by $d$ degrees, $f^{*}$ denotes the inverse image functor, $f_{!}$denotes direct image with compact support, $D: \mathscr{D}(X) \rightarrow \mathscr{D}(X)$ denotes the Verdier duality map, and ${ }^{p} H^{i}()$ denotes perverse cohomology sheaves. 
Objects of $\mathscr{D}(X)$ are referred to as complexes. The constant $l$-adic sheaf $\bar{Q}_{l}$ on any algebraic variety will be denoted 1 .

A complex $L$ in $\mathscr{D}(X)$ is said to be semisimple if $L$ is isomorphic to the direct sum $\bigoplus_{i}{ }^{p} H^{i} L[-i]$ and if each ${ }^{p} H^{i} L$ is a semisimple perverse sheaf.

Assume that we are given an action of a connected algebraic group $G$ on $X$. A semisimple complex $L$ in $\mathscr{D}(X)$ is said to be $G$-equivariant if each ${ }^{p} H^{i} L$ is a $G$-equivariant perverse sheaf (see $[\mathrm{L1}, 1.9]$ ).

2.2. Let $\mathbf{V} \in \mathscr{V}_{\nu}$. For each $(\mathbf{i}, \mathbf{a}) \in S_{\nu}$, we set $L_{\mathbf{i}, \mathbf{a} ; \Omega}=\left(\pi_{\mathbf{i}, \mathbf{a}}\right)_{1}(1) \in \mathscr{D}\left(\mathbf{E}_{\mathbf{V}, \Omega}\right)$. (Here, $1 \in \mathscr{D}\left(\widetilde{\mathscr{F}}_{\mathbf{i}, \mathbf{a}}\right)$; see 1.5.) By the decomposition theorem [BBD], $L_{\mathbf{i}, \mathbf{a} ; \Omega}$ is a semisimple complex.

We denote by $\mathscr{P}_{\mathbf{v}, \Omega}$ the set of isomorphism classes of simple perverse sheaves $L$ on $\mathbf{E}_{\mathbf{v}, \Omega}$ that have the following property: $L[d]$ appears as direct summand of $L_{\mathbf{i}, \mathbf{a} ; \Omega}$ for some $(\mathbf{i}, \mathbf{a}) \in S_{\nu}$ and some $d \in \mathbf{Z}$.

We denote by $\mathscr{Q}_{\mathbf{v}, \Omega}$ the subcategory of $\mathscr{D}\left(\mathbf{E}_{\mathbf{v}, \Omega}\right)$ consisting of all complexes that are isomorphic to finite direct sums of complexes of the form $L\left[d^{\prime}\right]$ for various $L \in \mathscr{P}_{\mathbf{v}, \Omega}$ and various integers $d^{\prime}$. Any complex in $\mathscr{Q}_{\mathbf{v}, \Omega}$ is semisimple. From 1.6(e) and [L1, (1.9.2)] it follows that any complex in $\mathscr{Q}_{\mathrm{V}, \Omega}$ is $G_{\mathbf{v}}$-equivariant.

If in (i, a) $\in S_{\nu}$ we have $a_{l}=0$ for some $l$ then by omitting $i_{l}$ from $\mathbf{i}$ and $a_{l}$ from $\mathbf{a}$, we obtain another element $\left(\mathbf{i}^{\prime}, \mathbf{a}^{\prime}\right) \in S_{\nu}$, and it is clear from the definition that $L_{\mathbf{i}, \mathbf{a} ; \Omega}=L_{\mathbf{i}^{\prime}, \mathbf{a}^{\prime} ; \Omega}$. Hence in the definition of $\mathscr{P}_{\mathbf{V}, \Omega}$ we may add the condition that

(a) $(\mathbf{i}, \mathbf{a})$ is such that $a_{l}>0$ for all $l$

and we obtain the same class of perverse sheaves. Since there are only finitely many elements of $S_{\nu}$ satisfying (a), we see that

(b) $\mathscr{P}_{\mathbf{V}, \Omega}$ contains only finitely many objects (up to isomorphism).

2.3. Assume that $\mathbf{V}, \tilde{\mathbf{V}}$ are in $\mathscr{V}_{\nu}$ and choose an isomorphism $l: \mathbf{V} \cong \tilde{\mathbf{V}}$ preserving the grading. This induces an isomorphism $\imath^{\prime}: \mathbf{E}_{\mathbf{V}, \Omega} \cong \mathbf{E}_{\widetilde{\mathbf{v}}, \Omega}$ given by $\imath^{\prime}(x)=\tilde{x}$ where $(\tilde{x})_{h}=l_{h^{\prime \prime}} x_{h^{\prime}} l_{h^{\prime}}^{-1}$ for all $h \in H$. Then $l_{!}^{\prime}: \mathscr{D}\left(\mathbf{E}_{\mathbf{v}, \Omega}\right) \rightarrow \mathscr{D}\left(\mathbf{E}_{\widetilde{\mathbf{v}}, \Omega}\right)$ is an equivalence of categories, with inverse $\imath^{\prime *}$; it carries $L_{\mathbf{i}, \mathbf{a} ; \Omega} \in \mathscr{D}\left(\mathbf{E}_{\mathbf{V}, \Omega}\right)$ to the analogous complex in $\mathscr{D}\left(\mathbf{E}_{\tilde{\mathbf{v}}, \Omega}\right)$, for each $(\mathbf{i}, \mathbf{a}) \in S_{\nu}$. Hence it defines an isomorphism $\mathscr{P}_{\mathbf{V}, \Omega} \cong \mathscr{P}_{\widetilde{\mathbf{v}}, \Omega}$ and an equivalence of categories $\mathscr{Q}_{\mathbf{v}, \Omega} \cong \mathscr{Q}_{\widetilde{\mathbf{v}}, \Omega}$ (due essentially to the equivariance of the complexes involved).

2.4. Assume again that $\mathbf{V} \in \mathscr{V}_{\nu}$. Let $(\mathbf{i}, \mathbf{a}) \in S_{\nu}$ be such that for two consecutive indices $l, l+1$ we have $i_{l}=i_{l+1}=i$. Let $\left(\mathbf{i}^{\prime}, \mathbf{a}^{\prime}\right) \in S_{\nu}$ be obtained from $(\mathbf{i}, \mathbf{a})$ by replacing the two entries $i_{l}, i_{l+1}$ by the single entry $i$ and the two entries $a_{l}, a_{l+1}$ by the single entry $a_{l}+a_{l+1}$. We then have

$$
L_{\mathbf{i}, \mathbf{a} ; \Omega} \cong \sum_{\kappa} L_{\mathbf{i}^{\prime}, \mathbf{a}^{\prime} ; \Omega}[-2 f(\kappa)] \in \mathscr{D}\left(\mathbf{E}_{\mathbf{v}, \Omega}\right)
$$

where $\kappa$ runs over the set of all sequences $1 \leq k_{1}<k_{2}<\cdots<k_{t} \leq a_{l}+$ $a_{l+1}$ and $f(\kappa)=\sum_{t=1}^{a_{l}}\left(k_{t}-t\right)$. This follows from the well-known structure of 
the cohomology of the Grassmannian of $a_{l+1}$-planes in $\left(a_{l}+a_{l+1}\right)$-dimensional space. As a consequence, we see that, in the definition of $\mathscr{P}_{\mathbf{v}, \Omega}$ we may add the condition that

(b) (i, a) is such that no two consecutive entries of $\mathbf{i}$ are equal.

\section{Multiplication}

3.1. Assume now that we are given $\mathbf{V}, \mathbf{v}^{\prime}, \mathbf{v}^{\prime \prime} \in \mathscr{V}$ such that $\mathbf{V} \in \mathscr{V}_{\nu}, \mathbf{v}^{\prime} \in$ $\mathscr{V}_{\nu^{\prime}}, \mathbf{V}^{\prime \prime} \in \mathscr{V}_{\nu^{\prime \prime}}$, where $\nu, \nu^{\prime}, \nu^{\prime \prime} \in \mathbf{N}^{I}$ satisfy $\nu=\nu^{\prime}+\nu^{\prime \prime}$.

Consider the diagram

$$
\mathbf{E}_{\mathbf{V}^{\prime}, \Omega} \times \mathbf{E}_{\mathbf{V}^{\prime \prime}, \Omega} \stackrel{p_{1}}{\longleftarrow} \mathbf{E}^{\prime} \stackrel{p_{2}}{\longrightarrow} \mathbf{E}^{\prime \prime} \stackrel{p_{3}}{\longrightarrow} \mathbf{E}_{\mathbf{V}, \Omega}
$$

where the notations are as follows.

$\mathbf{E}^{\prime \prime}$ is the variety of all pairs $(x, V)$ where $x \in \mathbf{E}_{\mathbf{v}, \Omega}$ and $V$ is an $x$-stable $I$-graded subspace of $\mathbf{V}$ such that $V \in \mathscr{V}_{\nu^{\prime \prime}}$.

$\mathbf{E}^{\prime}$ is the variety of all quadruples $\left(x, V, R^{\prime \prime}, R^{\prime}\right)$ where $(x, V) \in \mathbf{E}^{\prime \prime}, R^{\prime \prime}$ is an isomorphism $\mathbf{V}^{\prime \prime} \cong V$ (in $\mathscr{V}$ ) and $R^{\prime}$ is an isomorphism $\mathbf{V}^{\prime} \cong \mathbf{V} / V$ (in $\mathscr{V})$.

We have $p_{1}\left(x, V, R^{\prime \prime}, R^{\prime}\right)=\left(x^{\prime}, x^{\prime \prime}\right)$ where $x_{h} R_{h^{\prime}}^{\prime}=R_{h^{\prime \prime}}^{\prime} x_{h}^{\prime}: \mathbf{V}_{h^{\prime}}^{\prime} \rightarrow \mathbf{V}_{h^{\prime \prime}} / V_{h^{\prime \prime}}$ and $x_{h} R_{h^{\prime}}^{\prime \prime}=R_{h^{\prime \prime}}^{\prime \prime} x_{h}^{\prime \prime}: \mathbf{V}_{h^{\prime}}^{\prime \prime} \rightarrow V_{h^{\prime \prime}}$ for all $h \in H$;

$p_{2}\left(x, V, R^{\prime \prime}, R^{\prime}\right)=(x, V), p_{3}(x, V)=x$.

Note that $p_{1}$ is smooth with connected fibres, $p_{2}$ is a $G_{\mathbf{v}^{\prime}} \times G_{\mathbf{v}^{\prime \prime}}$-principal bundle and $p_{3}$ is proper.

Let $L^{\prime} \in \mathscr{D}\left(\mathbf{E}_{\mathbf{V}^{\prime}, \Omega}\right)$ and let $L^{\prime \prime} \in \mathscr{D}\left(\mathbf{E}_{\mathbf{v}^{\prime \prime}, \Omega}\right)$ be two semisimple complexes; assume that $L^{\prime}$ is $G_{\mathbf{V}^{\prime}}$-equivariant and that $L^{\prime \prime}$ is $G_{\mathbf{V}^{\prime \prime}}$-equivariant. We shall associate to $L^{\prime}, L^{\prime \prime}$ a complex $L=L^{\prime} * L^{\prime \prime}$ in $\mathscr{D}\left(\mathbf{E}_{\mathbf{v}, \Omega}\right)$ by the method of [L2, 9.5].

Let $L_{1}=L^{\prime} \otimes L^{\prime \prime} \in \mathscr{D}\left(\mathbf{E}_{\mathbf{v}^{\prime}, \Omega} \times \mathbf{E}_{\mathbf{v}^{\prime \prime}, \Omega}\right.$ ) (external tensor product). Let $L_{2}=$ $p_{1}^{*}\left(L_{1}\right)$; this is a $G_{\mathbf{V}^{\prime}} \times G_{\mathbf{V}^{\prime \prime}}$-equivariant semisimple complex on $\mathbf{E}^{\prime}$, and hence there is a well-defined semisimple complex $L_{3}$ on $\mathbf{E}^{\prime \prime}$ such that $p_{2}^{*}\left(L_{3}\right) \cong L_{2}$. We define $L=L^{\prime} * L^{\prime \prime}=\left(p_{3}\right)_{!} L_{3} \in \mathscr{D}\left(\mathbf{E}_{\mathbf{v}, \Omega}\right)$.

Lemma 3.2. (a) In the setup of 3.1 , we assume that $L^{\prime} \in \mathscr{Q}_{\mathbf{V}^{\prime}, \Omega}$ and $L^{\prime \prime} \in \mathscr{Q}_{\mathbf{V}^{\prime \prime}, \Omega}$. Then we have $L^{\prime} * L^{\prime \prime} \in \mathscr{Q}_{\mathbf{v}, \Omega}$.

(b) Assume that $\left(\mathbf{i}^{\prime}, \mathbf{a}^{\prime}\right) \in S_{\nu^{\prime}} \quad\left(\right.$ resp. $\left.\quad\left(\mathbf{i}^{\prime \prime}, \mathbf{a}^{\prime \prime}\right) \in S_{\nu^{\prime \prime}}\right)$. Let $\mathbf{i}=\mathbf{i}^{\prime} \mathbf{i}^{\prime \prime} \quad$ (resp. $\mathbf{a}=\mathbf{a}^{\prime} \mathbf{a}^{\prime \prime}$ ) be the sequence formed by the sequence $\mathbf{i}^{\prime}$ (resp. $\mathbf{a}^{\prime}$ ) followed by the sequence $\mathbf{i}^{\prime \prime}$ (resp. $\mathbf{a}^{\prime \prime}$ ). Then $(\mathbf{i}, \mathbf{a}) \in S_{\nu}$. Hence $L_{\mathbf{i}^{\prime}, \mathbf{a}^{\prime} ; \Omega} \in \mathscr{Q}_{\mathbf{V}^{\prime}, \Omega}, L_{\mathbf{i}^{\prime \prime}, \mathbf{a}^{\prime \prime} ; \Omega} \in$ $\mathscr{Q}_{\mathbf{V}^{\prime \prime}, \Omega}, L_{\mathbf{i}, \mathbf{a} ; \Omega} \in \mathscr{Q}_{\mathbf{v}, \Omega}$ are well defined. We have $L_{\mathbf{i}^{\prime}, \mathbf{a}^{\prime} ; \Omega} * L_{\mathbf{i}^{\prime \prime}, \mathbf{a}^{\prime \prime} ; \Omega}=L_{\mathbf{i}, \mathbf{a} ; \Omega}$.

We first prove (b). Let $\mathbf{i}^{\prime}=\left(i_{1}^{\prime}, \ldots, i_{m^{\prime}}^{\prime}\right), \mathbf{i}^{\prime \prime}=\left(i_{1}^{\prime \prime}, \ldots, i_{m^{\prime \prime}}^{\prime \prime}\right)$, so that $\mathbf{i}=$ $\left(i_{1}^{\prime}, \ldots, i_{m^{\prime}}^{\prime}, i_{1}^{\prime \prime}, \ldots, i_{m^{\prime \prime}}^{\prime \prime}\right)$. Let $L^{\prime}=L_{\mathbf{i}^{\prime}, \mathbf{a}^{\prime} ; \Omega}, L^{\prime \prime}=L_{\mathbf{i}^{\prime \prime}, \mathbf{a}^{\prime \prime} ; \Omega}, L=L_{\mathbf{i}, \mathbf{a} ; \Omega}$. Recall that $L^{\prime}=\left(\pi_{\mathbf{i}^{\prime}, \mathbf{a}^{\prime}}\right)_{!}(1) \in \mathscr{D}\left(\mathbf{E}_{\mathbf{V}^{\prime}, \Omega}\right), L^{\prime \prime}=\left(\pi_{\mathbf{i}^{\prime \prime}, \mathbf{a}^{\prime \prime}}\right)_{!}(1) \in \mathscr{D}\left(\mathbf{E}_{\mathbf{V}^{\prime \prime}, \Omega}\right), L=$ $\left(\pi_{\mathbf{i}, \mathbf{a}}\right)_{!}(1) \in \mathscr{D}\left(\mathbf{E}_{\mathbf{V}, \Omega}\right)$, where $\pi_{\mathbf{i}^{\prime}, \mathbf{a}^{\prime}}: \widetilde{\mathscr{F}}_{\mathbf{i}^{\prime}, \mathbf{a}^{\prime}} \rightarrow \mathbf{E}_{\mathbf{V}^{\prime}, \Omega}, \pi_{\mathbf{i}^{\prime \prime}, \mathbf{a}^{\prime \prime}}: \widetilde{\mathscr{F}_{\mathbf{i}^{\prime \prime}}, \mathbf{a}^{\prime \prime}} \rightarrow \mathbf{E}_{\mathbf{V}^{\prime \prime}, \Omega}$, $\pi_{\mathbf{i}, \mathbf{a}}: \widetilde{F}_{\mathbf{i}, \mathbf{a}} \rightarrow \mathbf{E}_{\mathbf{V}, \Omega}$, are as in 1.5. We apply the definitions of 3.1 to $L^{\prime}, L^{\prime \prime}$. In 
our case we have $L_{1}=\left(\pi_{\mathbf{i}^{\prime}, \mathbf{a}^{\prime}} \times \pi_{\mathbf{i}^{\prime}, \mathbf{a}^{\prime}}\right)(1)$ where $\pi_{\mathbf{i}^{\prime}, \mathbf{a}^{\prime}} \times \pi_{\mathbf{i}^{\prime}, \mathbf{a}^{\prime}}: \widetilde{\mathscr{F}}_{\mathbf{i}^{\prime}, \mathbf{a}^{\prime}} \times \widetilde{\mathscr{F}}_{\mathbf{i}^{\prime \prime}, \mathbf{a}^{\prime \prime}} \rightarrow$ $\mathbf{E}_{\mathbf{V}^{\prime}, \Omega} \times \mathbf{E}_{\mathbf{V}^{\prime \prime}, \Omega}$.

Let $\rho: \mathscr{\mathscr { F }}_{\mathrm{i}, \mathrm{a}} \rightarrow \mathbf{E}^{\prime \prime}$ be the morphism defined by $\rho(x, \phi)=(x, V)$ where $\phi=\left(\mathbf{V}=\mathbf{V}^{0} \supset \mathbf{V}^{1} \supset \cdots \supset \mathbf{V}^{m^{\prime}+m^{\prime \prime}}=0\right)$ and $V=\mathbf{V}^{m^{\prime}}$. Let $L_{3}=\rho_{!}(1) \epsilon$ $\mathscr{D}\left(\mathbf{E}^{\prime \prime}\right)$. As in 1.6(d), we see that $\rho$ is a proper morphism; using also 1.6(c) and the decomposition theorem [BBD], we see that $L_{3}$ is a semisimple complex on $\mathbf{E}^{\prime \prime}$. It is clear that $p_{2}^{*}\left(L_{3}\right)=p_{1}^{*}\left(L_{1}\right)$ (notations of 3.1). It is also clear that $p_{3} \rho=\pi_{\mathbf{i}, \mathbf{a}} ;$ hence

$$
L^{\prime} * L^{\prime \prime}=\left(p_{3}\right)_{!}\left(L_{3}\right)=\left(p_{3}\right)_{!} \rho_{!}(1)=\left(\pi_{\mathbf{i}, \mathbf{a}}\right)_{!}(1)=L .
$$

Thus, (b) is verified.

Now (a) follows immediately from (b).

3.3. We want to prove an associativity property of the operation $*$. Assume that we are given $\mathbf{v}^{123}, \mathbf{v}^{12}, \mathbf{v}^{23}, \mathbf{v}^{1}, \mathbf{v}^{2}, \mathbf{V}^{3}$ in $\mathscr{V}_{\nu(123)}, \mathscr{V}_{\nu(12)}, \mathscr{V}_{\nu(23)}, \mathscr{V}_{\nu(1)}$, $\mathscr{V}_{\nu(2)}, \mathscr{V}_{\nu(3)}$ respectively where $\nu(12)=\nu(1)+\nu(2), \nu(23)=\nu(2)+\nu(3)$ and $\nu(123)=\nu(1)+\nu(2)+\nu(3)$. Let $L^{f} \in \mathscr{Q}_{\mathbf{v}^{f}, \Omega}, f=1,2,3$. Then $L^{1} * L^{2} \in$ $\mathscr{Q}_{\mathbf{V}^{12}, \Omega}, L^{2} * L^{3} \in \mathscr{Q}_{\mathbf{v}^{23}, \Omega} ;$ hence $L^{1} *\left(L^{2} * L^{3}\right) \in \mathscr{Q}_{\mathbf{v}^{123}, \Omega}$ and $\left(L^{1} * L^{2}\right) * L^{3} \in$ $\mathscr{Q}_{\mathbf{V}^{123}, \Omega}$ are well defined (see 3.2(a)).

Lemma 3.4. $L^{1} *\left(L^{2} * L^{3}\right) \cong\left(L^{1} * L^{2}\right) * L^{3}$ in $\mathscr{Q}_{\mathbf{V}^{123}}$.

Consider the diagram analogous to that in $3.1(\mathrm{a})$ :

$$
\mathbf{E}_{\mathbf{V}^{1}, \Omega} \times \mathbf{E}_{\mathbf{V}^{2}, \Omega} \times \mathbf{E}_{\mathbf{V}^{3}, \Omega} \stackrel{p_{1}}{\longleftarrow} X \stackrel{p_{2}}{\longrightarrow} Y \stackrel{p_{3}}{\longrightarrow} \mathbf{E}_{\mathbf{V}^{123}, \Omega}
$$

where the notation is as follows.

$Y$ is the variety of all triples $\left(x^{123}, V^{3}, V^{23}\right)$ where $x^{123} \in \mathbf{E}_{\mathbf{v}^{123}, \Omega}, V^{3}, V^{23}$ are $x^{123}$-stable $I$-graded subspaces of $\mathbf{V}^{123}$ such that $V^{3} \subset V^{23}, V^{3}$, is isomorphic to $\mathbf{V}^{3}$, and $V^{23}$ is isomorphic to $\mathbf{V}^{23}$.

$X$ is the variety of all sequences $\left(x^{123}, V^{3}, V^{23}, R^{1}, R^{2}, R^{3}\right)$ where $\left(x^{123}, V^{3}, V^{23}\right) \in Y, R^{3}$ is an isomorphism $\mathbf{V}^{3} \cong V^{3}, R^{2}$ is an isomorphism $\mathbf{V}^{2} \cong V^{23} / V^{3}$, and $R^{1}$ is an isomorphism $\mathbf{V}^{1} \cong \mathbf{V}^{123} / V^{23}$.

We have $p_{1}\left(x^{123}, V^{3}, V^{23}, R^{1}, R^{2}, R^{3}\right)=\left(x^{1}, x^{2}, x^{3}\right)$ where $x_{h}^{123} R_{h^{\prime}}^{f}=$ $R_{h^{\prime \prime}}^{f} x_{h}^{f}$ for all $h \in H$ and $f=1,2,3$;

$p_{2}\left(x^{123}, V^{3}, V^{23}, R^{1}, R^{2}, R^{3}\right)=\left(x^{123}, V^{3}, V^{23}\right)$;

$p_{3}\left(x^{123}, V^{3}, V^{23}\right)=x^{123}$.

Let $L_{1}=L^{1} \otimes L^{2} \otimes L^{3}$ (a semisimple complex in $\mathscr{D}\left(\mathbf{E}_{\mathbf{v}^{1}, \Omega} \times \mathbf{E}_{\mathbf{V}^{2}, \Omega} \times \mathbf{E}_{\mathbf{V}^{3}, \Omega}\right)$. As in 3.1, there is a well-defined semisimple complex $L_{3} \in \mathscr{D}(Y)$ such that $p_{1}^{*} L_{1}=p_{2}^{*} L_{3}$. We set $L=\left(p_{3}\right)_{!} L_{2} \in \mathscr{D}\left(\mathbf{E}_{\mathbf{v}^{123}, \Omega}\right)$.

It is sufficient to show that

$$
L \cong L^{1} *\left(L^{2} * L^{3}\right), \quad L \cong\left(L^{1} * L^{2}\right) * L^{3} .
$$


These isomorphisms are established by using the standard commutation relation between inverse image and direct image with compact support in cartesian diagrams (base change). We leave the details to the reader.

3.5. As a consequence of 3.4 , it makes sense to consider $L^{1} * L^{2} * \cdots * L^{m} \in$ $\mathscr{Q}_{\mathbf{V}, \Omega}$ for any $L^{l} \in \mathscr{Q}_{\mathbf{W}^{l}, \Omega}, \mathbf{W}^{l} \in \mathscr{V}_{\nu^{l}}(l=1, \ldots, m)$ where $\mathbf{V} \in \mathscr{V}_{\nu}$ satisfies $\nu=\nu^{1}+\nu^{2}+\cdots+\nu^{m}$. This may be defined either directly, as in the proof of 3.4 , or by applying repeatedly the definition in 3.1 (for two factors) with some choice of brackets; the result is then independent of the choice of brackets.

3.6. Let $i \in I$ and let $a \in \mathbf{N}$. Let $\mathbf{W} \in \mathscr{V}$ be such that $\operatorname{dim} \mathbf{W}_{i}=a$ and $\mathbf{W}_{j}=0$ for all $j \neq i$. Then $\mathbf{E}_{\mathbf{v}, \Omega}=0$. It is clear that $L_{i, a ; \Omega}=1 \in \mathscr{D}(\mathbf{W}, \Omega)$. (Here we regard $i, a$ as sequences with one term each.) It follows that

$$
1 \in \mathscr{P}_{\mathbf{W}, \Omega} \text {. }
$$

3.7. Let $(\mathbf{i}, \mathbf{a}) \in S_{\nu}$. Let $\nu^{l}$ be such that $\nu^{l}\left(i_{l}\right)=a_{l}$ and $\nu^{l}(i)=0$ for $i \neq i_{l}$ $(i=1, \ldots, m)$ where $m$ is the number of terms in i. Let $\mathbf{W}^{l} \in \mathscr{V}_{\nu^{\prime}}$ and let $L^{l}=1 \in D_{\mathbf{w}^{l}, \Omega}$ for $l=1, \ldots, m$.

Assume that $m \geq 1$. Let $\mathbf{V}^{\prime \prime} \in \mathscr{V}_{\nu-\nu^{1}}$ and let $\left(\mathbf{i}^{\prime \prime}, \mathbf{a}^{\prime \prime}\right) \in S_{\nu-\nu^{1}}$ be obtained from (i, a) by dropping the first terms $i_{1}, a_{1}$. From 3.2(b) we see that

$$
L_{\mathbf{i}, \mathbf{a} ; \Omega}=L^{1} * L_{\mathbf{i}^{\prime}, \mathbf{a}^{\prime} ; \Omega} \in \mathscr{Q}_{\mathbf{V} ; \Omega} .
$$

Applying (a) repeatedly we obtain

$$
L_{\mathbf{i}, \mathbf{a} ; \Omega}=L^{1} * L^{2} * \cdots * L^{m} \in \mathscr{Q}_{\mathbf{v} ; \Omega} .
$$

Note that $L^{l} \in \mathscr{P}_{\mathbf{w}^{l}, \Omega}$, see 3.6.

3.8. The Verdier duality map $D: \mathscr{D}\left(\mathbf{E}_{\mathbf{V}, \Omega}\right) \rightarrow \mathscr{D}\left(\mathbf{E}_{\mathbf{v}, \Omega}\right)$ satisfies

$$
D\left(L_{\mathbf{i}, \mathbf{a} ; \Omega}\right)=L_{\mathbf{i}, \mathbf{a} ; \Omega}[2 d(\mathbf{i}, \mathbf{a})]
$$

where $d(\mathbf{i}, \mathbf{a})=\operatorname{dim} \widetilde{\mathscr{F}_{\mathbf{i}}, \mathbf{a}}$ is equal to

$$
\sum_{l^{\prime} \leq l} \sharp\left\{h \in \Omega \mid h^{\prime}=i_{l^{\prime}}, h^{\prime \prime}=i_{l}\right\} a_{l^{\prime}} a_{l}+\sum_{l<l^{\prime}: i_{l^{\prime}}=i_{l}} a_{l^{\prime}} a_{l}
$$

(see 1.6(c)). It follows that $D$ preserves the subcategory $\mathscr{Q}_{\mathrm{V}, \Omega}$ and that it defines a permutation of the set $\mathscr{P}_{\mathbf{V}, \Omega}$.

If $L^{\prime} \in \mathscr{Q}_{\mathbf{V}^{\prime}, \Omega}, L^{\prime \prime} \in \mathscr{Q}_{\mathbf{V}^{\prime}, \Omega}$ are as in 3.2(a), we have

$$
D\left(L^{\prime} * L^{\prime \prime}\right)=D\left(L^{\prime}\right) * D\left(L^{\prime \prime}\right)[2 m],
$$

where $m$ is the dimension of any fibre of $p_{1}$ minus the dimension of any fibre of $p_{2}$ (notation of 3.1 ); in other words, we have

$$
m=\sum_{h \in \Omega} \nu_{h^{\prime}}^{\prime} \nu_{h^{\prime \prime}}^{\prime \prime}+\sum_{i} \nu_{i}^{\prime} \nu_{i}^{\prime \prime} .
$$


Note also that

$$
D(L[d])=D(L)[-d]
$$

for any $L \in \mathscr{Q}_{\mathbf{V}}$ and any integer $d$.

\section{RESTRICTION}

4.1. Assume now that we are given $\mathbf{V} \in \mathscr{V}_{\nu}$ and an $I$-graded subspace $\mathbf{W}$ of $\mathbf{V}$ with $\mathbf{W} \in \mathscr{V}_{\omega}$. Let $\mathbf{T}=\mathbf{V} / \mathbf{W}$. Then $\mathbf{T} \in \mathscr{V}_{\tau}$ where $\nu=\tau+\omega$.

Let $E(\mathbf{W})$ be the subspace of $\mathbf{E}_{\mathbf{V}, \Omega}$ consisting of all $x=\left(x_{h}\right)$ such that $\mathbf{W}$ is $x$-stable. Such $x$ induces elements $x^{\prime} \in \mathbf{E}_{\mathbf{T}, \Omega}$ and $x^{\prime \prime} \in \mathbf{E}_{\mathbf{W}, \Omega}$ (see 1.3). We denote by $i: E(\mathbf{W}) \rightarrow \mathbf{E}_{\mathbf{v}, \Omega}$ the canonical inclusion and by $p: E(\mathbf{W}) \rightarrow$ $\mathbf{E}_{\mathbf{T}, \Omega} \times \mathbf{E}_{\mathbf{W}, \Omega}$ the map defined by $p(x)=\left(x^{\prime}, x^{\prime \prime}\right)$ where $x^{\prime}, x^{\prime \prime}$ are as above.

A complex $L \in \mathscr{D}\left(\mathbf{E}_{\mathbf{T}, \Omega} \times \mathbf{E}_{\mathbf{W}, \Omega}\right)$ is said to belong to $\mathscr{Q}_{\mathbf{T}, \mathbf{w}, \Omega}$ if there exist $L_{1}^{\prime}, \ldots, L_{s}^{\prime}$ in $\mathscr{Q}_{\mathrm{T}, \Omega}$ and $L_{1}^{\prime \prime}, \ldots, L_{s}^{\prime \prime}$ in $\mathscr{Q}_{\mathrm{W}, \Omega}$ such that we have $L \cong$ $\bigoplus_{j=1}^{s} L_{j}^{\prime} \otimes L_{j}^{\prime \prime}$ (external tensor product) in $\mathscr{D}\left(\mathbf{E}_{\mathbf{T}, \Omega} \times \mathbf{E}_{\mathbf{W}, \Omega}\right)$.

For any $L \in \mathscr{D}\left(\mathbf{E}_{\mathbf{v}, \Omega}\right)$, we set

$$
\operatorname{res}_{\mathbf{T}, \mathbf{W}} L=p_{!}\left(i^{*} L\right) \in \mathscr{D}\left(\mathbf{E}_{\mathbf{T}, \Omega} \times \mathbf{E}_{\mathbf{W}, \Omega}\right) \text {. }
$$

Proposition 4.2. (a) If $L \in \mathscr{Q}_{\mathrm{V}, \Omega}$, then $\operatorname{res}_{\mathrm{T}, \mathrm{w}} L$ belongs to $\mathscr{Q}_{\mathrm{T}, \mathrm{W}, \Omega}$.

(b) If $(\mathbf{i}, \mathbf{a}) \in S_{\nu}$, then

$$
\operatorname{res}_{\mathbf{T}, \mathbf{w}} L_{\mathbf{i}, \mathbf{a} ; \Omega} \cong \bigoplus_{\mathbf{a}^{\prime}, \mathbf{a}^{\prime \prime}} L_{\mathbf{i}, \mathbf{a}^{\prime} ; \Omega} \otimes L_{\mathbf{i}, \mathbf{a}^{\prime \prime} ; \Omega}\left[-2 M\left(\mathbf{a}^{\prime}, \mathbf{a}^{\prime \prime}\right)\right]
$$

where the summation is over the pairs of sequences $\mathbf{a}^{\prime}, \mathbf{a}^{\prime \prime}$ such that $\left(\mathbf{i}, \mathbf{a}^{\prime}\right) \in S_{\tau}$, $\left(\mathbf{i}, \mathbf{a}^{\prime \prime}\right) \in S_{\omega}$ and $\mathbf{a}^{\prime}+\mathbf{a}^{\prime \prime}=\mathbf{a}$; we have

$$
M\left(\mathbf{a}^{\prime}, \mathbf{a}^{\prime \prime}\right)=\sum_{l^{\prime} \leq l} \sharp\left\{h \in \Omega \mid h^{\prime}=i_{l^{\prime}}, h^{\prime \prime}=i_{l}\right\} a_{l^{\prime}}^{\prime} a_{l}^{\prime \prime}+\sum_{l<l^{\prime}: i_{l^{\prime}}=i_{l}} a_{l^{\prime}}^{\prime} a_{l}^{\prime \prime} .
$$

It is clear that (b) implies (a). Hence it is enough to prove (b). The proof will be given in 4.8 .

4.3. Let $\pi_{\mathbf{i}, \mathbf{a}}: \widetilde{F}_{\mathbf{i}, \mathbf{a}} \rightarrow \mathbf{E}_{\mathbf{V}, \Omega}$ be as in 1.5. The inverse image of $E(\mathbf{W})$ under $\pi_{\mathbf{i}, \mathbf{a}}$ is denoted $\widetilde{E}(\mathbf{W})$; let $\tilde{\pi}: \widetilde{E}(\mathbf{W}) \rightarrow E(\mathbf{W})$ be the restriction $\pi_{\mathbf{i}, \mathbf{a}}$. Consider the composition $p \tilde{\pi}: \widetilde{E}(\mathbf{W}) \rightarrow \mathbf{E}_{\mathbf{T}, \Omega} \times \mathbf{E}_{\mathbf{W}, \Omega}$. We have clearly

$$
p_{!}\left(i^{*}\left(L_{\mathbf{i}, \mathbf{a} ; \Omega}\right)\right)=(p \tilde{\pi})_{!}(1) .
$$

We now define, for any $\mathbf{a}^{\prime}, \mathbf{a}^{\prime \prime}$ as in $4.2(\mathrm{~b})$, some subvarieties $\widetilde{E}\left(\mathbf{W}, \mathbf{a}^{\prime}, \mathbf{a}^{\prime \prime}\right)$ of $\widetilde{E}(\mathbf{W})$ as follows.

$\widetilde{E}\left(\mathbf{W}, \mathbf{a}^{\prime}, \mathbf{a}^{\prime \prime}\right)$ is the variety of all $(x, \phi) \in \widetilde{E}(\mathbf{W})$ where $\phi=\left(\mathbf{V}=\mathbf{V}^{0} \supset\right.$ $\left.\mathbf{V}^{1} \supset \cdots \supset \mathbf{V}^{m}=0\right)$ is such that for any $l,\left(\mathbf{V}^{l-1} \cap \mathbf{W}\right) /\left(\mathbf{V}^{l} \cap \mathbf{W}\right)$ has dimension $a_{l}^{\prime \prime}$ in degree $i_{l}$ (and, necessarily, dimension 0 in degrees $\neq i_{l}$ ); here $m$ is the number of terms in $\mathbf{i}$.

It is clear that the $\widetilde{E}\left(\mathbf{W}, \mathbf{a}^{\prime}, \mathbf{a}^{\prime \prime}\right)$ form a partition of $\widetilde{E}(\mathbf{W})$ into locally closed subvarieties. 
For $\mathbf{a}^{\prime}, \mathbf{a}^{\prime \prime}$ as above, the morphisms $\pi_{\mathbf{i}, \mathbf{a}^{\prime}}: \widetilde{\mathscr{F}}_{\mathbf{i}, \mathbf{a}^{\prime}} \rightarrow \mathbf{E}_{\mathbf{T}, \Omega}$ and $\pi_{\mathbf{i}, \mathbf{a}^{\prime \prime}}: \widetilde{\mathscr{F}}_{\mathbf{i}, \mathbf{a}^{\prime \prime}} \rightarrow$ $\mathbf{E}_{\mathbf{W}, \Omega}$ can be defined just as in 1.5 (with $\mathbf{V}$ replaced by $\mathbf{T}, \mathbf{W}$ ). Moreover, we have a commutative diagram

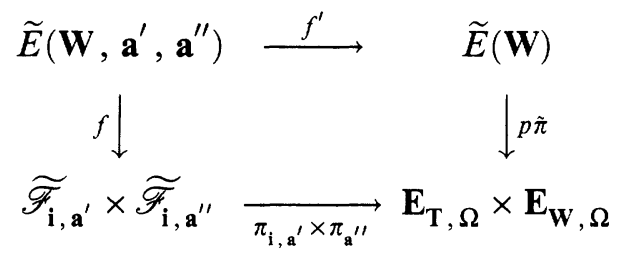

where $f^{\prime}$ is the inclusion and $f$ is the morphism defined as follows. If $(x, \phi) \in \widetilde{E}\left(\mathbf{W}, \mathbf{a}^{\prime}, \mathbf{a}^{\prime \prime}\right)$ is as above, then $f(x, \phi)=\left(\left(x^{\prime}, \phi^{\prime}\right),\left(x^{\prime \prime}, \phi^{\prime \prime}\right)\right)$ where $\left(x^{\prime}, x^{\prime \prime}\right)=p(x), \phi^{\prime \prime}$ is given by the intersections of the subspaces in $\phi$ with $\mathbf{W}$ and $\phi^{\prime}$ is given by the images of the subspaces in $\phi$ under the canonical projection $\mathbf{V} \rightarrow \mathbf{T}$.

Lemma 4.4. With the notations in 4.3, $f$ is a (locally trivial) vector bundle with fibres of dimension $M\left(\mathbf{a}^{\prime}, \mathbf{a}^{\prime \prime}\right)$ (see 4.2(b)).

We fix $\left(x^{\prime}, \phi^{\prime}\right) \in \widetilde{\mathscr{F}}_{\mathbf{i}, \mathbf{a}^{\prime}}$ and $\left(x^{\prime \prime}, \phi^{\prime \prime}\right) \in \widetilde{\mathscr{F}}_{\mathbf{i}, \mathbf{a}^{\prime \prime}}$ where $\phi^{\prime}=\left(\mathbf{T}=\mathbf{T}^{0} \supset \mathbf{T}^{1} \supset\right.$ $\left.\cdots \supset \mathbf{T}^{m}=0\right), \phi^{\prime \prime}=\left(\mathbf{W}=\mathbf{W}^{0} \supset \mathbf{W}^{1} \supset \cdots \supset \mathbf{W}^{m}=0\right)$. Let $\Gamma$ be the fibre of $f$ at $\left(\left(x^{\prime}, \phi^{\prime}\right),\left(x^{\prime \prime}, \phi^{\prime \prime}\right)\right)$. Let us identify $\mathbf{T}$ with a (graded) complement of $\mathbf{W}$ in $\mathbf{V}$.

Now, giving a graded subspace $\mathbf{V}^{l}$ of $\mathbf{V}$ such that $\mathbf{V}^{l} \cap \mathbf{W}=\mathbf{W}^{l}$ and such that the image of $\mathbf{V}^{l}$ under the canonical projection $\mathbf{V} \rightarrow \mathbf{T}$ is $\mathbf{T}^{l}$ is the same as giving a graded linear map $z_{l}: \mathbf{T}^{l} \rightarrow \mathbf{W} / \mathbf{W}^{l}$. (To $z_{l}$ corresponds the subspace $\mathbf{V}^{l}$ of $\mathbf{V}$ consisting of all vectors $v^{\prime}+v^{\prime \prime} \in \mathbf{T} \oplus \mathbf{W}$ such that $v^{\prime} \in \mathbf{T}^{l}$ and $z_{l}\left(v^{\prime}\right)=v^{\prime \prime}$ modulo $\mathbf{W}^{l}$.) The condition that the subspace $\mathbf{V}^{l}$ corresponding to $z_{l}$ (as above) is contained in the subspace $\mathbf{V}^{l-1}$ corresponding to $z_{l-1}: \mathbf{T}^{l-1} \rightarrow \mathbf{W} / \mathbf{W}^{l-1}$ (as above, with $l$ replaced by $l-1$ ) is that

(a) for any $v^{\prime} \in \mathbf{T}^{l}$, we have $z_{l}\left(v^{\prime}\right)=z_{l-1}\left(v^{\prime}\right)$ as elements of $\mathbf{W} / \mathbf{W}^{l-1}$ $(2 \leq l \leq m)$.

We then have automatically that $\mathbf{V}^{l-1} / \mathbf{V}^{l}$ is zero in degrees $\neq i_{l}$ and has dimension $a_{l}$ in degree $i_{l}$.

Now, giving an element $x \in \mathbf{E}_{\mathbf{V}, \Omega}$ such that $p(x)=\left(x^{\prime}, x^{\prime \prime}\right)$ is the same as giving an element

(b) $y=\left(y_{h}\right) \in \bigoplus_{h \in H} \operatorname{Hom}\left(\mathbf{T}_{h^{\prime}}, \mathbf{W}_{h^{\prime \prime}}\right)$ such that $y_{h}=0$ whenever $h \notin \Omega$.

(To $y$ corresponds $x$ such that $x_{h}\left(v^{\prime \prime}\right)=x_{h}^{\prime \prime}\left(v^{\prime \prime}\right)$ and $x_{h}\left(v^{\prime}\right)=x_{h}^{\prime}\left(v^{\prime}\right)+$ $y_{h}\left(v^{\prime}\right)$ for all $\left.h \in H, v^{\prime} \in \mathbf{T}_{h^{\prime}}, v^{\prime \prime} \in \mathbf{W}_{h^{\prime}}.\right)$

The condition that such $x$ satisfies $x_{h}\left(\mathbf{V}_{h^{\prime}}^{l}\right) \subset \mathbf{V}_{h^{\prime \prime}}^{l}$ (where $\mathbf{V}^{l}$ corresponds to $z_{l}$ as above) is that

(c) $z_{l, h^{\prime \prime}} x_{h}^{\prime}-x_{h}^{\prime \prime} z_{l, h^{\prime}}-y_{h}: \mathbf{T}_{h^{\prime}}^{l} \rightarrow \mathbf{W}_{h^{\prime \prime}} / \mathbf{W}_{h^{\prime \prime}}^{l}$ is zero for any $h \in H$, where $z_{l, i}$ is the $i$-component of $z_{l}$. (This equality has a meaning since $x_{h}^{\prime}\left(v^{\prime}\right) \in \mathbf{T}_{h^{\prime \prime}}^{l}$ and $x_{h}^{\prime \prime}$ induces a linear map $\mathbf{W}_{h^{\prime}} / \mathbf{W}_{h^{\prime}}^{l} \rightarrow \mathbf{W}_{h^{\prime \prime}} / \mathbf{W}_{h^{\prime \prime}}^{l}$, denoted again $x_{h}^{\prime \prime}$.) 
We thus see that $\Gamma$ can be identified with the $\mathbf{k}$-vector space $\Gamma^{\prime}$ consisting of all $\left(z_{1}, \ldots, z_{m} ; y\right)$ where the graded linear maps $z_{l}: \mathbf{T}^{l} \rightarrow \mathbf{W} / \mathbf{W}^{l}(1 \leq l \leq$ $m$ ) and $y$ as in (b) are subject to (a) and (c). We have a natural short exact sequence $0 \rightarrow \Gamma_{1}^{\prime} \rightarrow \Gamma^{\prime} \rightarrow \Gamma_{2}^{\prime} \rightarrow 0$ where $\Gamma_{1}^{\prime}$ consists of all $y$ as in (b) such that $y_{h}\left(\mathbf{T}_{h^{\prime}}^{l}\right) \subset \mathbf{W}_{h^{\prime \prime}}^{l}$ for all $h, l$ and $\Gamma_{2}^{\prime}$ consists of all $\left(z_{1}, \ldots, z_{m}\right)$ where $z_{l}: \mathbf{T}^{l} \rightarrow \mathbf{W} / \mathbf{W}^{l}$ are graded linear maps satisfying (a). Clearly,

$$
\operatorname{dim} \Gamma_{1}^{\prime}=\sum_{l^{\prime} \leq l ; h \in \Omega} \operatorname{dim}\left(\mathbf{T}_{h^{\prime}}^{l^{\prime}-1} / \mathbf{T}_{h^{\prime}}^{l^{\prime}}\right) \operatorname{dim}\left(\mathbf{W}_{h^{\prime \prime}}^{l-1} / \mathbf{W}_{h^{\prime \prime}}^{l}\right)
$$

hence, using the definition of $\mathbf{a}^{\prime}, \mathbf{a}^{\prime \prime}$, we have

$$
\operatorname{dim} \Gamma_{1}^{\prime}=\sum_{l^{\prime} \leq l} \sharp\left\{h \in \Omega \mid h^{\prime}=i_{l^{\prime}}, h^{\prime \prime}=i_{l}\right\} a_{l^{\prime}}^{\prime} a_{l}^{\prime \prime} .
$$

On the other hand,

$$
\operatorname{dim} \Gamma_{2}^{\prime}=\sum_{l<l^{\prime} ; i \in I} \operatorname{dim}\left(\mathbf{T}_{i}^{l^{\prime}-1} / \mathbf{T}_{i}^{l^{\prime}}\right) \operatorname{dim}\left(\mathbf{W}_{i}^{l-1} / \mathbf{W}_{i}^{l}\right)
$$

hence

$$
\operatorname{dim} \Gamma_{2}^{\prime}=\sum_{l<l^{\prime}: i_{l^{\prime}}=i_{l}} a_{l^{\prime}}^{\prime} a_{l}^{\prime \prime}
$$

We have $\operatorname{dim} \Gamma^{\prime}=\operatorname{dim} \Gamma_{1}^{\prime}+\operatorname{dim} \Gamma_{2}^{\prime}=M\left(\mathbf{a}^{\prime}, \mathbf{a}^{\prime \prime}\right)$.

The local triviality statement is left to the reader.

Lemma 4.5. In the setup of 4.4, we have

$$
\left(p \tilde{\pi} f^{\prime}\right)_{!}(1) \cong L_{\mathbf{i}, \mathbf{a}^{\prime} ; \Omega} \otimes L_{\mathbf{i}, \mathbf{a}^{\prime \prime} ; \Omega}\left[-2 M\left(\mathbf{a}^{\prime}, \mathbf{a}^{\prime \prime}\right)\right] .
$$

This follows from 4.4 and the commutative diagram in 4.3.

4.6. We can find a sequence $\left(Z_{j}\right)$ of closed subsets of $\widetilde{E}(\mathbf{W})(j \in \mathbf{Z})$ with the following properties: $Z_{j-1} \subset Z_{j}$ for all $j, Z_{j}=\widetilde{E}(\mathbf{W})$ for large $j, Z_{j}$ is empty for $j<0$, and each difference $Z_{j}-Z_{j-1}$ is a union of subvarieties of the form $\widetilde{E}\left(\mathbf{W}, \mathbf{a}^{\prime}, \mathbf{a}^{\prime \prime}\right.$ ) (see 4.3), which are both open and closed in $Z_{j}-Z_{j-1}$.

If $\beta_{j}$ is the inclusion $Z_{j} \subset \widetilde{E}(\mathbf{W})$ and $\gamma_{j}$ is the inclusion $Z_{j}-Z_{j-1} \subset \widetilde{E}(\mathbf{W})$, then we have a canonical distinguished triangle in $\mathscr{D}\left(\mathbf{E}_{\mathbf{T}, \Omega} \times \mathbf{E}_{\mathbf{W}, \Omega}\right)$ :

$$
\left((p \tilde{\pi})_{!}\left(\gamma_{j}\right)_{!} \gamma_{j}^{*}(1),(p \tilde{\pi})_{!}\left(\beta_{j}\right)_{!} \beta_{j}^{*}(1)(p \tilde{\pi})_{!}\left(\beta_{j-1}\right)_{!} \beta_{j-1}^{*}(1)\right) .
$$

It gives rise to a long exact sequence of perverse cohomomology sheaves

(a) $\quad \cdots \rightarrow{ }^{p} H^{s-1}\left((p \tilde{\pi})_{!}\left(\beta_{j-1}\right)_{!} \beta_{j-1}^{*}(1)\right)$

$$
\stackrel{\delta}{\rightarrow}{ }^{p} H^{S}\left((p \tilde{\pi})_{!}\left(\gamma_{j}\right)_{!} \gamma_{j}^{*}(1)\right) \rightarrow{ }^{p} H^{s}\left((p \tilde{\pi})_{!}\left(\beta_{j}\right)_{!} \beta_{j}^{*}(1)\right) \rightarrow \ldots
$$

Note that

(b)

$(p \tilde{\pi})_{!}\left(\beta_{j}\right)_{!} \beta_{j}^{*}(1)$ is $p_{!}\left(i^{*}\left(L_{\mathbf{i}, \mathbf{a}}\right)\right)$ for large $j($ see $4.3(\mathrm{a}))$, and is zero for $j<0$. 
Lemma 4.7. (a) For each integer $j$, the maps $\delta$ in 4.6(a) are zero.

(b) For each integer $j$, the complex $(p \tilde{\pi})_{!}\left(\beta_{j}\right)_{!} \beta_{j}^{*}(1)$ in $\mathscr{D}\left(\mathbf{E}_{\mathbf{T}, \Omega} \times \mathbf{E}_{\mathbf{W}, \Omega}\right)$ is semisimple; it is isomorphic to the direct sum

$$
\bigoplus_{\mathbf{a}^{\prime}, \mathbf{a}^{\prime \prime}} L_{\mathbf{i}, \mathbf{a}^{\prime} ; \Omega} \otimes L_{\mathbf{i}, \mathbf{a}^{\prime \prime} ; \Omega}\left[-2 M\left(\mathbf{a}^{\prime}, \mathbf{a}^{\prime \prime}\right)\right]
$$

where the summation is over the pairs of sequences $\mathbf{a}^{\prime}, \mathbf{a}^{\prime \prime}$ as in 4.2 with $\widetilde{E}\left(\mathbf{W}, \mathbf{a}^{\prime}, \mathbf{a}^{\prime \prime}\right) \subset Z_{j}$.

The proof will go along the lines of [L1, 3.7]. Assuming that (a) and the first assertion of (b) are proved, we prove the second assertion of (b) as follows. Since both complexes in question are semisimple, it is enough to prove that they have the same ${ }^{p} H^{s}$ for any $s$. From 4.5 we see that

$$
(p \tilde{\pi})_{!}\left(\gamma_{j}\right)_{!} \gamma_{j}^{*}(1) \cong \bigoplus_{\mathbf{a}^{\prime}, \mathbf{a}^{\prime \prime}} L_{\mathbf{i}, \mathbf{a}^{\prime} ; \Omega} \otimes L_{\mathbf{i}, \mathbf{a}^{\prime \prime} ; \Omega}\left[-2 M\left(\mathbf{a}^{\prime}, \mathbf{a}^{\prime \prime}\right)\right]
$$

where the summation is over the pairs of sequences $\mathbf{a}^{\prime}, \mathbf{a}^{\prime \prime}$ as in 4.2 with $\widetilde{E}\left(\mathbf{W}, \mathbf{a}^{\prime}, \mathbf{a}^{\prime \prime}\right) \subset Z_{j}-Z_{j-1}$. In particular, $(p \tilde{\pi})_{!}\left(\gamma_{j}\right)_{!} \gamma_{j}^{*}(1)$ is semisimple. Using (a), we see that $4.6(\mathrm{a})$ decomposes into short exact sequences of semisimple perverse sheaves. Hence, ${ }^{p} H^{s}\left((p \tilde{\pi})_{!}\left(\beta_{j}\right)_{!} \beta_{j}^{*}(1)\right)$ is isomorphic to

$$
{ }^{p} H^{s}\left((p \tilde{\pi})_{!}\left(\beta_{j-1}\right)_{!} \beta_{j-1}^{*}(1)\right) \oplus^{p} H^{s}\left((p \tilde{\pi})_{!}\left(\gamma_{j}\right) ! \gamma_{j}^{*}(1)\right) \text {. }
$$

Using the last formula, together with (c) and an induction on $j$ we obtain the desired equality for ${ }^{p} H^{s}$. (The case where $j<0$ is trivial, by $4.6(\mathrm{~b})$.)

It remains to prove (a) and the first assertion of (b). By general principles [BBD, $\S 6]$, it is enough to prove them in the case where the ground field is an algebraic closure of the finite field $F_{q}$ with $q$ elements. In this case, we can realize $4.6(\mathrm{a})$ in the category of mixed perverse sheaves over an $F_{q}$-form of $\mathbf{E}_{\mathbf{T}, \Omega} \times \mathbf{E}_{\mathbf{W}, \Omega}$. The isomorphism (c) remains valid in this category (with the same proof) except that after the shift $\left[-2 M\left(\mathbf{a}^{\prime}, \mathbf{a}^{\prime \prime}\right)\right]$ one should add the Tate twist $\left(-M\left(\mathbf{a}^{\prime}, \mathbf{a}^{\prime \prime}\right)\right)$. By Deligne's theorem [D] $L_{\mathbf{i}, \mathbf{a}^{\prime} ; \Omega} \otimes L_{\mathbf{i}, \mathbf{a}^{\prime \prime} ; \Omega}$ is a pure complex of weight zero; after applying to it the shift and the twist just described, it remains pure of weight zero (see [BBD, 6.1.4]). Hence, by (c),

$$
(p \tilde{\pi})_{!}\left(\gamma_{j}\right)_{!} \gamma_{j}^{*}(1) \text { is a pure complex of weight zero. }
$$

It follows that ${ }^{p} H^{s}\left((p \tilde{\pi})_{!}\left(\gamma_{j}\right)_{!} \gamma_{j}^{*}(1)\right)$ is pure of weight $s$.

We now show by induction on $j$ that ${ }^{p} H^{s}\left((p \tilde{\pi})_{!}\left(\beta_{j}\right)_{!} \beta_{j}^{*}(1)\right)$ is a pure complex of weight $s$. This is obvious for $j<0$, by $4.6(\mathrm{~b})$. If we assume that this is true for $j-1$, the statement for $j$ follows from 4.6(a), using (d), the statement for $j-1$, and the following fact: if $L_{1} \rightarrow L_{2} \rightarrow L_{3}$ is an exact sequence of mixed perverse sheaves with $L_{1}, L_{3}$ pure of weight $s$, then $L_{2}$ is also pure of weight $s$.

Now, using [BBD, 5.4.4], it follows that $(p \tilde{\pi})_{!}\left(\beta_{j}\right)_{!} \beta_{j}^{*}(1)$ is pure of weight zero. Using the decomposition theorem $[\mathrm{BBD}, 5.4 .5,5.3 .8]$ it follows that 
$(p \tilde{\pi})_{!}\left(\beta_{j}\right)_{!} \beta_{j}^{*}(1)$ is semisimple. The vanishing of $\delta$ in $4.6(\mathrm{a})$ follows from the fact that $\delta$ is a morphism between two perverse sheaves of different weights. This completes the proof of the lemma.

4.8. We now note that (in view of 4.6(b)), 4.2(a) is a special case of $4.7(b)$ (for large $j$ ). This completes the proof of 4.2 .

4.9. We want to prove an associativity property of the operation res in 4.1.

Assume that we are given $\mathbf{V} \in \mathscr{V}$ and two $I$-graded subspaces $\mathbf{W}, \mathbf{W}^{\prime}$ of $\mathbf{V}$ such that $\mathbf{W} \subset \mathbf{W}^{\prime}$. We introduce some notation.

Consider the commutative diagram

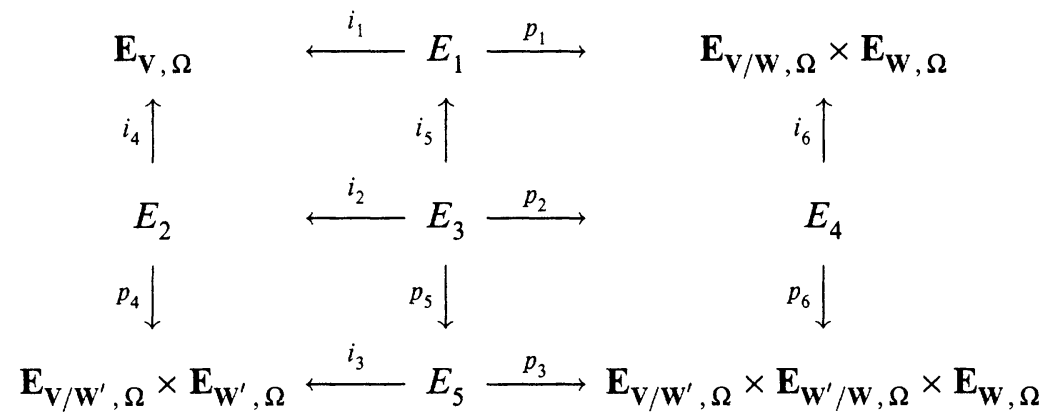

where the notation is as follows.

$E_{1}$ is the subspace of $\mathbf{E}_{\mathbf{v}, \Omega}$ consisting of all $x$ such that $\mathbf{W}$ is $x$-stable.

$E_{2}$ is the subspace of $\mathbf{E}_{\mathbf{v}, \Omega}$ consisting of all $x$ such that $\mathbf{W}^{\prime}$ is $x$-stable.

$E_{3}$ is the subspace of $\mathbf{E}_{\mathbf{v}, \Omega}$ consisting of all $x$ such that $\mathbf{W}$ and $\mathbf{W}^{\prime}$ are $x$-stable.

$E_{4}$ is the variety of all pairs $\left(y, y^{\prime}\right)$ in $\mathbf{E}_{\mathbf{v} / \mathbf{W}, \Omega} \times \mathbf{E}_{\mathbf{W}, \Omega}$ such that $\mathbf{W}^{\prime} / \mathbf{W}$ is $y$-stable.

$E_{5}$ is the variety of all pairs $\left(z, z^{\prime}\right)$ in $\mathbf{E}_{\mathbf{v} / \mathbf{W}^{\prime}, \Omega} \times \mathbf{E}_{\mathbf{W}^{\prime}, \Omega}$ such that $\mathbf{W}$ is $z^{\prime}$-stable.

$i_{1}, \ldots, i_{6}$ are the natural inclusions and $p_{1}, \ldots, p_{6}$ are the obvious projections.

We define a functor

$$
\operatorname{res}_{\mathbf{V} / \mathbf{w}^{\prime}, \mathbf{w}^{\prime} / \mathbf{w}}^{\prime}: \mathscr{D}\left(\mathbf{E}_{\mathbf{v} / \mathbf{w}, \Omega} \times \mathbf{E}_{\mathbf{w}, \Omega}\right) \rightarrow \mathscr{D}\left(\mathbf{E}_{\mathbf{V} / \mathbf{w}^{\prime}, \Omega} \times \mathbf{E}_{\mathbf{w}^{\prime} / \mathbf{w}, \Omega} \times \mathbf{E}_{\mathbf{w}, \Omega}\right)
$$

as the composition $\left(p_{6}\right) i_{6} i^{*}$.

We define a functor

$$
\operatorname{res}_{\mathbf{w}^{\prime} / \mathbf{w}, \mathbf{w}}^{\prime}: \mathscr{D}\left(\mathbf{E}_{\mathbf{v} / \mathbf{w}^{\prime}, \Omega} \times \mathbf{E}_{\mathbf{w}^{\prime}, \Omega}\right) \rightarrow \mathscr{D}\left(\mathbf{E}_{\mathbf{v} / \mathbf{w}^{\prime}, \Omega} \times \mathbf{E}_{\mathbf{w}^{\prime} / \mathbf{w}, \Omega} \times \mathbf{E}_{\mathbf{w}, \Omega}\right)
$$

as the composition $\left(p_{3}\right)_{!} i_{3}^{*}$.

From the definition we have

$$
\operatorname{res}_{\mathbf{V} / \mathbf{w}^{\prime}, \mathbf{w}^{\prime} / \mathbf{w}}^{\prime}\left(L_{1} \otimes L_{2}\right) \cong \operatorname{res}_{\mathbf{V} / \mathbf{w}^{\prime}, \mathbf{w}^{\prime} / \mathbf{w}}\left(L_{1}\right) \otimes L_{2}
$$

for any $L_{1} \in \mathscr{D}\left(\mathbf{E}_{\mathbf{v} / \mathbf{w}, \Omega}\right), L_{2} \in \mathscr{D}\left(\mathbf{E}_{\mathbf{w}, \Omega}\right)$ and

$$
\operatorname{res}_{\mathbf{W}^{\prime} / \mathbf{W}, \mathbf{W}}^{\prime}\left(L_{3} \otimes L_{4}\right)=L_{3} \otimes \operatorname{res}_{\mathbf{W}^{\prime} / \mathbf{W}, \mathbf{W}}\left(L_{4}\right)
$$


for any $L_{3} \in \mathscr{D}\left(\mathbf{E}_{\mathbf{V} / \mathbf{w}^{\prime}, \Omega}\right), L_{4} \in \mathscr{D}\left(\mathbf{E}_{\mathbf{w}^{\prime}, \Omega}\right)$ (external tensor products).

Proposition 4.10. Let $L \in \mathscr{D}\left(\mathbf{E}_{\mathbf{v}, \Omega}\right)$. In the setup of 4.9 , we have

$$
\operatorname{res}_{\mathbf{V} / \mathbf{w}^{\prime}, \mathbf{w}^{\prime} / \mathbf{w}}^{\prime} \operatorname{res}_{\mathbf{V} / \mathbf{w}, \mathbf{w}}(L) \cong \operatorname{res}_{\mathbf{w}^{\prime} / \mathbf{w}, \mathbf{w}}^{\prime} \operatorname{res}_{\mathbf{V} / \mathbf{w}^{\prime}, \mathbf{w}^{\prime}}(L) .
$$

We must show that (with notations of 4.9$)$ we have $\left(p_{6}\right) !_{6} i_{6}^{*}\left(p_{1}\right) i_{1}^{*}(L) \cong$ $\left(p_{3}\right) i_{3}^{*}\left(p_{4}\right) i_{4}^{*}(L)$. From the diagram in 4.9 we see that in the sequence

$$
\left(p_{6}\right)_{!} i_{6}^{*}\left(p_{1}\right)_{!} i_{1}^{*}(L) ;\left(p_{6}\right)_{!}\left(p_{2}\right)_{!} i_{5}^{*} i_{1}^{*}(L) ;\left(p_{3}\right)_{!}\left(p_{5}\right)_{!} i_{2}^{*} i_{4}^{*}(L) ;\left(p_{3}\right)_{i} i_{3}^{*}\left(p_{4}\right)_{!} i_{4}^{*}(L),
$$

any two consecutive complexes are isomorphic. The proposition is proved.

\section{FOURIER-DELIGNE TRANSFORM}

5.1. In this section we assume that $\mathbf{k}$ is an algebraic closure of a finite field $F_{q}$. We fix a nontrivial character $F_{q} \rightarrow \bar{Q}_{l}^{*}$. This defines an Artin-Schreier local system of rank 1 on $\mathbf{k}$; its inverse image under any morphism $T: X \rightarrow \mathbf{k}$ of algebraic varieties is a local system $\mathscr{L}_{T}$ of rank 1 on $X$.

5.2. Assume given two orientations $\Omega, \Omega^{\prime} \subset H$ for our graph. For any $\mathbf{V} \in \mathscr{V}$ we define $T: \mathbf{E}_{\mathbf{V}, \Omega \cup \Omega^{\prime}} \rightarrow \mathbf{k}$ by $T(x)=\sum_{h} \operatorname{tr}\left(x_{h} x_{\bar{h}}\right)$ where the sum is taken over all $h \in \Omega-\left(\Omega \cap \Omega^{\prime}\right)$. (The last trace is that of an endomorphism of $\mathbf{V}_{h^{\prime \prime}}$.) The function $T$ is $G_{\mathbf{V}}$-invariant. Hence the local system $\mathscr{L}_{T}$ on $\mathbf{E}_{\mathbf{V}, \Omega \cup \Omega^{\prime}}$ is well defined (see 5.1) and $G_{\mathbf{v}}$-equivariant.

We have two surjective linear maps

$$
\mathbf{E}_{\mathbf{V}, \Omega} \stackrel{\delta}{\longleftarrow} \mathbf{E}_{\mathbf{V}, \Omega \cup \Omega^{\prime}} \stackrel{\delta^{\prime}}{\longrightarrow} \mathbf{E}_{\mathbf{V}, \Omega^{\prime}}
$$

defined by $\delta(x)=y, \delta^{\prime}(x)=y^{\prime}$ where $y_{h}=x_{h}$ for $h \in \Omega, y_{h}=0$ for $h \notin \Omega, y_{h}^{\prime}=x_{h}$ for $h \in \Omega^{\prime}, y_{h}^{\prime}=0$ for $h \notin \Omega^{\prime}$.

Next, we consider the functor $\mathscr{F}: \mathscr{D}\left(\mathbf{E}_{\mathbf{v}, \Omega}\right) \rightarrow \mathbf{E}_{\mathbf{v}, \Omega^{\prime}}$ defined by $\mathscr{F}(L)=$ $\delta_{!}^{\prime}\left(\delta^{*}(L) \otimes \mathscr{L}_{T}\right)[D]$ where $D=\sum_{h} \operatorname{dim} \mathbf{V}_{h^{\prime}} \operatorname{dim} \mathbf{V}_{h^{\prime \prime}} \quad$ (sum over all $h \in \Omega$ $\left.\left(\Omega \cap \Omega^{\prime}\right)\right)$. This is a special case of the Fourier-Deligne transform from the derived category on a vector bundle to that on the dual vector bundle. (The vector bundles in question are $\mathbf{E}_{\mathbf{V}, \Omega} \rightarrow \mathbf{E}_{\mathbf{V}, \Omega \cap \Omega^{\prime}}$ and $\mathbf{E}_{\mathbf{V}, \Omega^{\prime}} \rightarrow \mathbf{E}_{\mathbf{v}, \Omega \cap \Omega^{\prime}} ; T$ may be used to identify one vector bundle with the dual of the other.)

5.3. We now fix $\mathbf{V} \in \mathscr{V}_{\nu}, \mathbf{V}^{\prime} \in \mathscr{V}_{\nu^{\prime}}$ and $\mathbf{V}^{\prime \prime} \in \mathscr{V}_{\nu^{\prime \prime}}$ such that $\nu=\nu^{\prime}+\nu^{\prime \prime}$. Assume that $L^{\prime} \in \mathscr{D}\left(\mathbf{E}_{\mathbf{V}^{\prime}, \Omega}\right)$ is a semisimple $G_{\mathbf{v}^{\prime}}$-equivariant complex and that $L^{\prime \prime} \in \mathscr{D}\left(\mathbf{E}_{\mathbf{V}^{\prime \prime}, \Omega}\right)$ is a semisimple $G_{\mathbf{V}^{\prime \prime}}$-equivariant complex. Then $L^{\prime} *$ $L^{\prime \prime} \in \mathscr{D}\left(\mathbf{E}_{\mathbf{v}, \Omega}\right)$ is well defined; hence $\mathscr{F}\left(L^{\prime} * L^{\prime \prime}\right) \in \mathscr{D}\left(\mathbf{E}_{\mathbf{v}, \Omega^{\prime}}\right)$ is well defined. On the other hand, $\mathscr{F}\left(L^{\prime}\right)$ (resp. $\mathscr{F}\left(L^{\prime \prime}\right)$ ) is a semisimple, $G_{\mathbf{v}^{\prime}}$-equivariant (resp. $G_{\mathbf{V}^{\prime \prime}}$-equivariant) complex in $\mathscr{D}\left(\mathbf{E}_{\mathbf{V}^{\prime}, \Omega^{\prime}}\right)$ (resp. $\left.\mathscr{D}\left(\mathbf{E}_{\mathbf{V}^{\prime \prime}, \Omega^{\prime}}\right)\right)$, by general properties of the Fourier-Deligne transform. Hence $\mathscr{F}\left(L^{\prime}\right) * \mathscr{F}\left(L^{\prime \prime}\right) \in \mathscr{D}\left(\mathbf{E}_{\mathbf{v}, \Omega^{\prime}}\right)$ is well defined. We have the following result. 
Theorem 5.4. In the setup of 5.3, we have

$$
\mathscr{F}\left(L^{\prime} * L^{\prime \prime}\right) \cong \mathscr{F}\left(L^{\prime}\right) * \mathscr{F}\left(L^{\prime \prime}\right)[C]
$$

in $\mathscr{D}\left(\mathbf{E}_{\mathbf{v}, \Omega^{\prime}}\right)$ where

$$
C=\sum_{h \in \Omega-\left(\Omega \cap \Omega^{\prime}\right)}\left(\nu^{\prime \prime}\left(h^{\prime}\right) \nu^{\prime}\left(h^{\prime \prime}\right)-\nu^{\prime}\left(h^{\prime}\right) \nu^{\prime \prime}\left(h^{\prime \prime}\right)\right) .
$$

We consider the commutative diagram

$$
\begin{aligned}
& X_{a} \stackrel{u_{b a}}{\longleftarrow} X_{b} \stackrel{u_{b c}}{\longrightarrow} X_{c} \stackrel{u_{c d}}{\longrightarrow} X_{d} \\
& u_{e b} \uparrow \quad u_{f c} \uparrow \quad u_{g d} \uparrow \\
& X_{e} \stackrel{u_{e f}}{\longrightarrow} X_{f} \stackrel{u_{f g}}{\longrightarrow} X_{g} \\
& u_{j a} \uparrow \quad u_{h e} \uparrow \quad u_{i f} \uparrow \\
& X_{h} \stackrel{u_{h i}}{\longrightarrow} X_{i} \\
& u_{h k} \downarrow \quad u_{i l} \downarrow \quad u_{g p} \downarrow \\
& X_{j} \stackrel{u_{k j}}{\longleftarrow} X_{k} \stackrel{u_{k l}}{\longrightarrow} X_{l} \\
& u_{k n} \downarrow \quad u_{l o} \downarrow \\
& X_{m} \stackrel{u_{m n}}{\stackrel{u_{n o}}{\longrightarrow}} X_{n} \stackrel{u_{o p}}{\longrightarrow} X_{p}
\end{aligned}
$$

in which the notations are as follows.

$X_{a}=\mathbf{E}_{\mathbf{V}^{\prime}, \Omega} \times \mathbf{E}_{\mathbf{v}^{\prime \prime}, \Omega}$.

$X_{c}$ is the variety of all pairs $(x, V)$ where $x \in \mathbf{E}_{\mathbf{V}, \Omega}$ and $V$ is an $x$-stable $I$-graded subspace of $\mathbf{V}$ such that $V \in \mathscr{V}_{\nu^{\prime \prime}}$.

$X_{b}$ is the variety of all quadruples $\left(x, V, R^{\prime \prime}, R^{\prime}\right)$ where $(x, V) \in X_{c}, R^{\prime \prime}$ is an isomorphism $\mathbf{V}^{\prime \prime} \cong V$ (in $\mathscr{V}$ ), and $R^{\prime}$ is an isomorphism $\mathbf{V}^{\prime} \cong \mathbf{V} / V$ (in $\mathscr{V})$.

$X_{d}=\mathbf{E}_{\mathbf{V}, \Omega}$.

$X_{m}=\mathbf{E}_{\mathbf{V}^{\prime}, \Omega^{\prime}} \times \mathbf{E}_{\mathbf{V}^{\prime \prime}, \Omega^{\prime}}$.

$X_{o}$ is the variety of all pairs $(y, V)$ where $y \in \mathbf{E}_{\mathbf{v}, \Omega^{\prime}}$ and $V$ is a $y$-stable $I$-graded subspace of $\mathbf{V}$ such that $V \in \mathscr{V}_{\nu^{\prime \prime}}$.

$X_{n}$ is the variety of all quadruples $\left(y, V, R^{\prime \prime}, R^{\prime}\right)$ where $(y, V) \in X_{o}, R$ is an isomorphism $\mathbf{V}^{\prime \prime} \cong V$ (in $\mathscr{V}$ ), and $R^{\prime}$ is an isomorphism $\mathbf{V}^{\prime} \cong \mathbf{V} / V$ (in $\mathscr{V})$.

$X_{p}=\mathbf{E}_{\mathbf{V}, \Omega^{\prime}}$

$X_{g}=\mathbf{E}_{\mathbf{v}, \Omega \cup \Omega^{\prime}}$.

$X_{f}$ is the variety of all triples $(z, V)$ where $z \in X_{g}, V$ is an $I$-graded subspace of $\mathbf{V}$ such that $V \in \mathscr{V}_{\nu^{\prime \prime}}$, and $V$ is $x$-stable where $x=\delta(z)$ (see 5.2). 
$X_{e}$ is the variety of all quadruples $\left(z, V, R^{\prime \prime}, R^{\prime}\right)$ where $(z, V) \in X_{f}, R^{\prime \prime}$ is an isomorphism $\mathbf{V}^{\prime \prime} \cong V$ (in $\mathscr{V}$ ), and $R^{\prime}$ is an isomorphism $\mathbf{V}^{\prime} \cong \mathbf{V} / V$ (in $\mathscr{V})$.

$X_{i}$ is the variety of all pairs $(z, V)$ where $z \in X_{g}$ and $V$ is a $z$-stable $I$-graded subspace of $\mathbf{V}$ such that $V \in \mathscr{V}_{\nu^{\prime \prime}}$.

$X_{h}$ is the variety of all sequences $\left(z, V, R^{\prime \prime}, R^{\prime}\right)$ in $X_{e}$ such that $(z, V) \in$ $X_{i}$.

$X_{j}=\mathbf{E}_{\mathbf{v}^{\prime}, \Omega \cup \Omega^{\prime}} \times \mathbf{E}_{\mathbf{v}^{\prime \prime}, \Omega \cup \Omega^{\prime}}$.

$X_{k}$ is the variety of all sequences $\left(x^{\prime}, x^{\prime \prime}, y, V, R^{\prime \prime}, R^{\prime}\right)$ such that $\left(y, V, R^{\prime \prime}, R^{\prime}\right) \in X_{n},\left(x^{\prime}, x^{\prime \prime}\right) \in X_{a}, R_{h^{\prime \prime}}^{\prime} x_{h}^{\prime}=y_{h} R_{h^{\prime}}^{\prime}: \mathbf{V}_{h^{\prime}}^{\prime} \rightarrow \mathbf{V}_{h^{\prime \prime}} / V_{h^{\prime \prime}}$, and $y_{h} R_{h^{\prime}}^{\prime \prime}=R_{h^{\prime \prime}}^{\prime \prime} x_{h}^{\prime \prime}: \mathbf{V}_{h^{\prime}}^{\prime \prime} \rightarrow V_{h^{\prime \prime}}$ for all $h \in \Omega \cap \Omega^{\prime}$.

$X_{l}$ is the variety of all sequences $\left(s^{\prime}, s^{\prime \prime}, y, V\right)$ where $(y, V) \in X_{o}$ and

$$
\begin{aligned}
& s^{\prime}=\left(s_{h}^{\prime}\right) \in \bigoplus_{h \in \Omega-\left(\Omega \cap \Omega^{\prime}\right)} \operatorname{Hom}\left(\mathbf{V}_{h^{\prime}} / V_{h^{\prime}}, \mathbf{V}_{h^{\prime \prime}} / V_{h^{\prime \prime}}\right), \\
& s^{\prime \prime}=\left(s_{h}^{\prime \prime}\right) \in \bigoplus_{h \in \Omega-\left(\Omega \cap \Omega^{\prime}\right)} \operatorname{Hom}\left(V_{h^{\prime}}, V_{h^{\prime \prime}}\right) .
\end{aligned}
$$

The maps $u_{b a}, u_{b c}, u_{c d}, u_{n m}, u_{n o}, u_{o p}$ are defined as in 3.1 .

The maps $u_{g d}, u_{g p}$ are $\delta$ and $\delta^{\prime}$ of 5.2; the maps $u_{j a}, u_{j m}$ are of the form $\delta \times \delta$ and $\delta^{\prime} \times \delta^{\prime}$.

The map $u_{e b}$ takes $\left(z, V, R^{\prime \prime}, R^{\prime}\right)$ to $\left(\delta(z), V, R^{\prime \prime}, R^{\prime}\right)$.

The map $u_{f c}$ takes $(z, V)$ to $(\delta(z), V)$.

The map $u_{e f}$ takes $\left(z, V, R^{\prime \prime}, R^{\prime}\right)$ to $(z, V)$.

The map $u_{f g}$ is the first projection.

The maps $u_{h e}, u_{i f}$ are the obvious imbeddings.

The map $u_{h i}$ takes $\left(z, V, R^{\prime \prime}, R^{\prime}\right)$ to $(z, V)$.

The map $u_{k j}$ takes $\left(x^{\prime}, x^{\prime \prime}, y, V, R^{\prime \prime}, R^{\prime}\right)$ to $\left(z^{\prime}, z^{\prime \prime}\right)$ where $R_{h^{\prime \prime}}^{\prime} z_{h}^{\prime}=$ $y_{h} R_{h^{\prime}}^{\prime}: \mathbf{V}_{h^{\prime}}^{\prime} \rightarrow \mathbf{V}_{h^{\prime \prime}} / V_{h^{\prime \prime}}, y_{h} R_{h^{\prime}}^{\prime \prime}=R_{h^{\prime \prime}}^{\prime \prime} z_{h}^{\prime \prime}: \mathbf{V}_{h^{\prime}}^{\prime \prime} \rightarrow V_{h^{\prime \prime}}$ for all $h \in \Omega^{\prime}$, and $z_{h}^{\prime}=$ $x_{h}^{\prime}, z_{h}^{\prime \prime}=x_{h}^{\prime \prime}$ for all $h \in \Omega$.

The map $u_{k l}$ takes $\left(x^{\prime}, x^{\prime \prime}, y, V, R^{\prime \prime}, R^{\prime}\right)$ to $\left(s^{\prime}, s^{\prime \prime}, y, V\right)$ where $R_{h^{\prime \prime}}^{\prime} x_{h}^{\prime}=$ $s_{h}^{\prime} R_{h^{\prime}}^{\prime}: \mathbf{V}_{h^{\prime}}^{\prime} \rightarrow \mathbf{V}_{h^{\prime \prime}} / V_{h^{\prime \prime}}$, and $s_{h}^{\prime \prime} R_{h^{\prime}}^{\prime \prime}=R_{h^{\prime \prime}}^{\prime \prime} x_{h}^{\prime \prime}: \mathbf{V}_{h^{\prime}}^{\prime \prime} \rightarrow V_{h^{\prime \prime}}$ for all $h \in \Omega-\left(\Omega \cap \Omega^{\prime}\right)$.

The map $u_{h k}$ takes $\left(z, V, R^{\prime \prime}, R^{\prime}\right)$ to $\left(x^{\prime}, x^{\prime \prime}, y, V, R^{\prime \prime}, R^{\prime}\right)$ where $y=$ $\delta^{\prime}(z)$ and $\left(x^{\prime}, x^{\prime \prime}\right)=u_{b a} u_{e b}\left(z, V, R^{\prime \prime}, R^{\prime}\right)$.

The map $u_{i l}$ takes $(z, V)$ to $\left(s^{\prime}, s^{\prime \prime}, y, V\right)$ where $y=\delta^{\prime}(z)$ and $s^{\prime}, s^{\prime \prime}$ are induced by $x=\delta(z)$.

The map $u_{k n}$ takes $\left(x^{\prime}, x^{\prime \prime}, y, V, R^{\prime \prime}, R^{\prime}\right)$ to $\left(y, V, R^{\prime \prime}, R^{\prime}\right)$.

The map $u_{l o}$ takes $\left(s^{\prime}, s^{\prime \prime}, y, V\right)$ to $(y, V)$.

Recall that in 5.2 we have defined a function $T: X_{g} \rightarrow \mathbf{k}$; the same construction applied to the two factors of $X_{j}$ gives two analogous functions on $X_{j}$ whose sum is again denoted $T: X_{j} \rightarrow \mathbf{k}$. It is easy to check that the compositions $T u_{f g} u_{e f} u_{h e}, T u_{f g} u_{i f} u_{h i}, T u_{k j} u_{h k}$ coincide as functions $X_{h} \rightarrow \mathbf{k}$ and 
that there is a unique function $X_{l} \rightarrow \mathbf{k}$ whose composition with $u_{i l}$ (resp. with $u_{k l}$ ) gives the function $X_{i} \rightarrow \mathbf{k}$ (resp. $X_{k} \rightarrow \mathbf{k}$ ) just considered.

Hence we can define local systems of rank 1 on

$$
X_{j}, X_{k}, X_{h}, X_{l}, X_{i}, X_{e}, X_{f}, X_{g}
$$

that are $\mathscr{L}_{T}$ on $X_{j}, X_{g}$ and correspond to each other under inverse image by

$$
u_{f g}, u_{e f}, u_{h e}, u_{i f}, u_{h i}, u_{k j}, u_{h k}, u_{k l}, u_{i l} \text {. }
$$

We shall denote each of these local systems by $\mathscr{L}$.

Let $L_{a}=L^{\prime} \otimes L^{\prime \prime} \in \mathscr{D}\left(X_{a}\right)$ (external tensor product). Let $L_{b}=u_{b a}^{*} L_{a} \in$ $\mathscr{D}\left(X_{b}\right)$; let $L_{c} \in \mathscr{D}\left(X_{c}\right)$ be the unique semisimple complex such that $u_{b c}^{*} L_{c}=$ $L_{b}$ and let $L_{d}=\left(u_{c d}\right)_{!} L_{c} \in \mathscr{D}\left(X_{d}\right)$. Let $L_{g}=u_{g d}^{*} L_{d} \in \mathscr{D}\left(X_{g}\right)$ and let $L_{p}=\left(u_{g p}\right)_{!}\left(L_{g} \otimes \mathscr{L}\right) \in \mathscr{D}\left(X_{p}\right)$.

By definition, we have $\mathscr{F}\left(L^{\prime} * L^{\prime \prime}\right)=L_{p}[D]$, where $D=\sum_{h} \nu\left(h^{\prime}\right) \nu\left(h^{\prime \prime}\right)$ (sum over all $\left.h \in \Omega-\left(\Omega \cap \Omega^{\prime}\right)\right)$.

Now let $L_{j}=u_{j a}^{*} L_{a} \in \mathscr{D}\left(X_{j}\right)$ and $L_{m}=\left(u_{j m}\right)_{!}\left(L_{j} \otimes \mathscr{L}\right) \in \mathscr{D}\left(X_{m}\right)$. This is a semisimple, $G_{\mathbf{v}^{\prime}} \times G_{\mathbf{v}^{\prime \prime}}$-equivariant complex. Let $L_{n}=u_{n m}^{*} L_{m} \in \mathscr{D}\left(X_{n}\right)$, and let $L_{o} \in \mathscr{D}\left(X_{o}\right)$ be the unique semisimple complex such that $u_{n o}^{*} L_{o}=L_{n}$. Let $L_{p}^{\prime}=\left(u_{o p}\right)_{!} L_{o} \in \mathscr{D}\left(X_{p}\right)$.

By definition, we have

$$
\mathscr{F}\left(L^{\prime}\right) * \mathscr{F}\left(L^{\prime \prime}\right)=L_{p}^{\prime}\left[D^{\prime}+D^{\prime \prime}\right]
$$

where $D^{\prime}=\sum_{h} \nu^{\prime}\left(h^{\prime}\right) \nu^{\prime}\left(h^{\prime \prime}\right), D^{\prime \prime}=\sum_{h} \nu^{\prime \prime}\left(h^{\prime}\right) \nu^{\prime \prime}\left(h^{\prime \prime}\right)$ (sum over all $h \in \Omega-$ $\left.\left(\Omega \cap \Omega^{\prime}\right)\right)$.

Hence it suffices to prove that

$$
L_{p} \cong L_{p}^{\prime}\left[D^{\prime}+D^{\prime \prime}-D+C\right]
$$

Let $L_{e}=u_{e b}^{*} L_{b} \in \mathscr{D}\left(X_{e}\right), L_{f}=u_{f c}^{*} L_{c} \in \mathscr{D}\left(X_{f}\right)$. Then $L_{f}$ is a semisimple complex (since $L_{c}$ is semisimple and $u_{f_{c}}$ is smooth with connected fibres) and $L_{e}=u_{e f}^{*} L_{f}$. Moreover, $\left(u_{f g}\right)_{!} L_{f}=L_{g}$ (since the diagram $u_{f c}, u_{c d}, u_{f g}, u_{g d}$ is cartesian). Hence we may go from $L_{a}$ to $L_{p}$ by the shorter chain $L_{e}=$ $\left(u_{b a} u_{e b}\right)^{*} L_{a}, L_{e}=u_{e f}^{*} L_{f}\left(L_{f}\right.$ semisimple $), L_{p}=\left(u_{g p} u_{f g}\right)_{!}\left(L_{f} \otimes \mathscr{L}\right)$.

Similarly, we may go from $L_{a}$ to $L_{p}^{\prime}$ by the shorter chain $L_{k}=\left(u_{j a} u_{k j}\right)^{*} L_{a} \in$ $\mathscr{D}\left(X_{k}\right), L_{k}=u_{k l}^{*} L_{l}\left(L_{l} \in \mathscr{D}\left(X_{l}\right)\right.$ semisimple $), L_{p}^{\prime}=\left(u_{o p} u_{l o}\right)_{!}\left(L_{l} \otimes \mathscr{L}\right)$.

Let $L_{h}=u_{h k}^{*} L_{k} \in \mathscr{D}\left(X_{h}\right), L_{i}=u_{i l}^{*} L_{l} \in \mathscr{D}\left(X_{i}\right)$. Note that $u_{i l}$ is a vector bundle with fibres of dimension $D_{0}=\sum_{h} \nu^{\prime}\left(h^{\prime}\right) \nu^{\prime \prime}\left(h^{\prime \prime}\right)$ (sum over all $h \in$ $\left.\Omega-\left(\Omega \cap \Omega^{\prime}\right)\right)$.

It follows that $L_{i}$ is semisimple (recall that $L_{l}$ is semisimple) and that $\left(u_{i l}\right)_{!} L_{i}=L_{l}\left[-2 D_{0}\right]$. Hence we have $\left(u_{o p} u_{l o} u_{i l}\right)_{!}\left(L_{i} \otimes \mathscr{L}\right)=L_{p}^{\prime}\left[-2 D_{0}\right]$. We have the identity $C=D-D^{\prime}-D^{\prime \prime}-2 D_{0}$. Hence it is enough to prove that

$$
L_{p} \cong L_{p}^{\prime}\left[-2 D_{0}\right]
$$


We have $u_{h i}^{*} L_{i}=L_{h}$ and it follows that we may go from $L_{a}$ to $L_{p}^{\prime}$ by the chain $L_{h}=\left(u_{j a} u_{k j} u_{h k}\right)^{*} L_{a} \in \mathscr{D}\left(X_{h}\right), L_{h}=u_{h i}^{*} L_{i}\left(L_{i} \in \mathscr{D}\left(X_{i}\right)\right.$ semisimple), $L_{p}^{\prime}\left[-2 D_{0}\right]=\left(u_{o p} u_{l o} u_{i l}\right)_{!}\left(L_{i} \otimes \mathscr{L}\right)$.

We now show that $u_{i f}^{*} L_{f}$ is semisimple. This is not obvious since $u_{i f}$ is not smooth; however, we have $u_{i f}^{*} L_{f}=\left(u_{f c} u_{i f}\right)^{*} L_{c}$ with $L_{c}$ semisimple and $u_{f c} u_{i f}$ a vector bundle. Our assertion follows.

Now both $u_{i f}^{*} L_{f}$ and $L_{i}$ are semisimple and they have the same inverse image $L_{h}$ under $u_{h i}$ (a smooth morphism with connected fibres). It follows that $u_{i f}^{*} L_{f} \cong L_{i}$. Since $u_{o p} u_{l o} u_{i l}=u_{g p} u_{f g} u_{i f}$ we see that $L_{p}^{\prime}\left[-2 D_{0}\right]=$ $\left(u_{g p} u_{f g} u_{i f}\right)_{!}\left(u_{i f}^{*}\left(L_{f} \otimes \mathscr{L}\right)\right)$.

We now see that (a1) would be a consequence of the following statement:

(c) $L_{f} \otimes \mathscr{L}$ and $\left(u_{i f}\right)_{!} u_{i f}^{*} L_{f} \otimes \mathscr{L}$ have the same image under $\left(u_{g p} u_{f g}\right)_{!}$. An equivalent statement is the following one:

(c1) if $u^{\prime}$ denotes the inclusion of $X_{f}-X_{i}$ into $X_{f}$ (as an open subset), then $\left(u_{g p} u_{f g} u^{\prime}\right)_{!}\left(u^{\prime *}\left(L_{f} \otimes \mathscr{L}\right)=0\right.$.

(We use the distinguished triangle associated with the partition $X_{f}=X_{i} \cup$ $\left(X_{f}-X_{i}\right)$.)

We now consider the commutative diagram

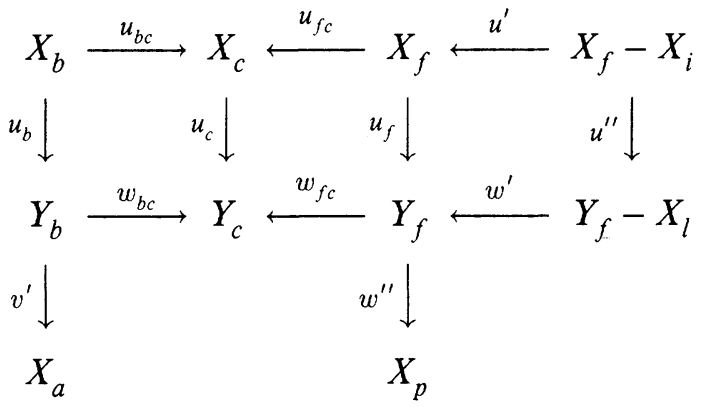

where the notations are as follows.

$Y_{c}$ is the variety of all triples $\left(s^{\prime}, s^{\prime \prime}, V\right)$ where $V$ is an $I$-graded subspace of $\mathbf{V}$ such that $V \in \mathscr{V}_{\nu^{\prime \prime}}, s^{\prime}=\left(s_{h}^{\prime}\right) \in \bigoplus_{h \in \Omega-\left(\Omega \cup \Omega^{\prime}\right)} \operatorname{Hom}\left(\mathbf{V}_{h^{\prime}} / V_{h^{\prime}}, \mathbf{V}_{h^{\prime \prime}} / V_{h^{\prime \prime}}\right)$, $s^{\prime \prime}=\left(s_{h}^{\prime \prime}\right) \in \bigoplus_{h \in \Omega-\left(\Omega \cup \Omega^{\prime}\right)} \operatorname{Hom}\left(V_{h^{\prime}}, V_{h^{\prime \prime}}\right)$.

$Y_{f}$ is the variety of all quadruples $\left(s^{\prime}, s^{\prime \prime}, y, V\right)$ where $\left(s^{\prime}, s^{\prime}, V\right) \in Y_{c}$ and $y \in Y_{p}$ satisfies $y_{h}\left(V_{h^{\prime}}\right) \subset V_{h^{\prime \prime}}$ for all $h \in \Omega \cap \Omega^{\prime}$.

$Y_{b}$ is the variety of all sequences $\left(s^{\prime}, s^{\prime \prime}, V, R^{\prime \prime}, R^{\prime}\right)$ where $\left(s^{\prime}, s^{\prime \prime}, V\right) \in Y_{c}$ and $R^{\prime \prime}, R^{\prime}$ are as in the definition of $X_{b}$.

The map $w_{b c}$ takes $\left(s^{\prime}, s^{\prime \prime}, V, R^{\prime \prime}, R^{\prime}\right)$ to $\left(s^{\prime}, s^{\prime \prime}, V\right)$.

The map $w_{f c}$ takes $\left(s^{\prime}, s^{\prime \prime}, y, V\right)$ to $\left(s^{\prime}, s^{\prime \prime}, V\right)$.

The map $w^{\prime}$ is the inclusion (as an open subset).

The map $u_{b}$ takes $\left(x, V, R^{\prime \prime}, R^{\prime}\right)$ to $\left(s^{\prime}, s^{\prime \prime}, V, R^{\prime \prime}, R^{\prime}\right)$ where $s^{\prime}, s^{\prime \prime}$ are induced by $x$.

The map $u_{c}$ takes $(x, V)$ to $\left(s^{\prime}, s^{\prime \prime}, V\right)$ where $s^{\prime}, s^{\prime \prime}$ are induced by $x$. 
The map $u_{f}$ takes $(z, V)$ to $\left(s^{\prime}, s^{\prime \prime}, y, V\right)$ where $y=\delta^{\prime}(z)$ and $s^{\prime}, s^{\prime \prime}$ are induced by $x=\delta(z)$.

The map $u^{\prime \prime}$ is the restriction of $u_{f}$.

The map $w^{\prime \prime}$ takes $\left(s^{\prime}, s^{\prime \prime}, y, V\right)$ to $y$.

The map $v^{\prime}$ is such that $v^{\prime} u_{b}=u_{b a}$.

Let $M_{b}=v^{\prime *} L_{a} \in \mathscr{D}\left(Y_{b}\right)$; this is a semisimple complex that is $G_{\mathbf{v}^{\prime}} \times G_{\mathbf{V}^{\prime \prime}}$ equivariant, and hence there is a well-defined semisimple complex $M_{c} \in \mathscr{D}\left(Y_{c}\right)$ such that $w_{b c}^{*} M_{c}=M_{b}$. Let $M_{f}=w_{f c}^{*} M_{c} \in \mathscr{D}\left(Y_{f}\right)$. It is clear that $u_{b}^{*} M_{b}=$ $L_{b}, u_{c}^{*} M_{c}=L_{c}, u_{f}^{*} M_{f}=L_{f}$ (note that $u_{b}, u_{c}, u_{f}$ are vector bundles). Hence we have $u^{\prime *} L_{f}=u^{\prime *} u_{f}^{*} M_{f}=u^{\prime \prime} * w^{\prime *} M_{f}$.

The statement (c1) can now be rewritten in terms of $M_{f}$ instead of $L_{f}$ :

(d) $\left(u_{g p} u_{f g} u^{\prime}\right)_{!}\left(u^{\prime \prime *} w^{\prime *} M_{f} \otimes \mathscr{L}\right)=0$.

or equivalently (using $u_{g p} u_{f g} u^{\prime}=w^{\prime \prime} w^{\prime} u^{\prime \prime}$ ):

$$
\left(w^{\prime \prime} w^{\prime} u^{\prime \prime}\right)_{!}\left(u^{\prime *} w^{\prime *} M_{f} \otimes \mathscr{L}\right)=0
$$

This would be a consequence of the following statement:

(e) $u_{!}^{\prime \prime}\left(u^{\prime \prime *} w^{\prime *} M_{f} \otimes \mathscr{L}\right)=0$.

We have $u_{!}^{\prime \prime}\left(u^{\prime \prime *} w^{\prime *} M_{f} \otimes \mathscr{L}\right)=w^{\prime *} M_{f} \otimes\left(u_{!}^{\prime \prime} \mathscr{L}\right)$; hence it suffices to prove that

(f) $u_{!}^{\prime \prime} \mathscr{L}=0$ in $\mathscr{D}\left(Y_{f}-X_{l}\right)$.

Let us fix a point $\left(s^{\prime}, s^{\prime \prime}, y, V\right) \in Y_{f}-X_{l}$ and let $\Gamma$ be the fibre of $w^{\prime}$ over this point. Let $\widetilde{T}: \Gamma \rightarrow \mathbf{k}$ be the restriction of $T u_{f g}$.

By base change, it is enough to prove that the cohomology with compact support of $\Gamma$ with coefficients in $\mathscr{L} \mid \Gamma$ is zero. Note that $\mathscr{L} \mid \Gamma$ is the local system defined as in 5.1 in terms of the function $\widetilde{T}$. Hence, by a known property of Artin-Schreier coverings, it would be enough to verify the following statement: one can identify $\Gamma$ with $\mathbf{k}^{N}$ for some $N$ so that $\widetilde{T}$ is given by a nonconstant affine linear form on $\mathbf{k}^{N}$.

Let us choose an $I$-graded subspace $W$ of $\mathbf{V}$ that is complementary to $V$. We have an isomorphism $\Gamma \cong \bigoplus_{h \in \Omega-\left(\Omega \cap \Omega^{\prime}\right)} \operatorname{Hom}\left(W_{h^{\prime}}, V_{h^{\prime \prime}}\right)$ given by $(z, V) \rightarrow z^{\prime}=\left(z_{h}^{\prime}\right)$ where $z_{h}^{\prime}$ are restrictions of $x=\delta(z)$. Let $y_{h}^{\prime}: V_{h^{\prime \prime}} \rightarrow W_{h^{\prime}}$ be the composition of the imbedding $V_{h^{\prime \prime}} \rightarrow \mathbf{V}_{h^{\prime \prime}}$ with $y_{\bar{h}}: \mathbf{V}_{h^{\prime \prime}} \rightarrow \mathbf{V}_{h^{\prime}}$ and with the projection $\mathbf{V}_{h^{\prime}} \rightarrow W_{h^{\prime}}$. A simple computation shows that, in the coordinates $\left(z_{h}^{\prime}\right)$, the function $\widetilde{T}$ is given by the expression $\sum_{h \in \Omega-\left(\Omega \cap \Omega^{\prime}\right)} \operatorname{tr}\left(z_{h}^{\prime} y_{h}^{\prime}\right)+$ constant . (The last trace is that of an endomorphism of $V_{h^{\prime \prime}}$.) We must show that the linear part of the last expression is not identically zero.

Assume that it is identically zero. (Recall that $y$ is fixed.) Then we have $y_{\bar{h}}^{\prime}=0$ for all $h \in \Omega-\left(\Omega \cap \Omega^{\prime}\right)$. In other words, we have $y_{\bar{h}}\left(V_{h^{\prime \prime}}\right) \subset V_{h^{\prime}}$ for all $h \in \Omega-\left(\Omega \cap \Omega^{\prime}\right)$. Hence $y_{h}\left(V_{h^{\prime}}\right) \subset V_{h^{\prime \prime}}$ for all $h \in \Omega-\left(\Omega \cap \Omega^{\prime}\right)$. The same inclusion holds for $h \in \Omega \cap \Omega^{\prime}$ by the definition of $Y_{f}$. It follows that $\left(s^{\prime}, s^{\prime \prime}, y, V\right) \in X_{l}$, a contradiction. The theorem is proved. 
5.5. Let

$$
m_{\Omega}\left(\nu^{\prime}, \nu^{\prime \prime}\right)=\sum_{h \in \Omega} \nu_{h^{\prime}}^{\prime} \nu_{h^{\prime \prime}}^{\prime \prime}+\sum_{i} \nu_{i}^{\prime} \nu_{i}^{\prime \prime}
$$

(see 3.8).

The integer $C$ in 5.4 can be expressed as follows:

$$
C=m_{\Omega^{\prime}}\left(\nu^{\prime}, \nu^{\prime \prime}\right)-m_{\Omega}\left(\nu^{\prime}, \nu^{\prime \prime}\right) \text {. }
$$

This is verified by an easy computation.

Corollary 5.6. (a) For any (i, a) $\in S_{\nu}$ we have

$$
\mathscr{F}\left(L_{\mathbf{i}, \mathbf{a} ; \Omega}\right) \cong L_{\mathbf{i}, \mathbf{a} ; \Omega^{\prime}}[d] \in \mathscr{D}\left(\mathbf{E}_{\mathbf{v}, \Omega^{\prime}}\right)
$$

for some integer $d$.

(b) $\mathscr{F}$ defines a bijection $\mathscr{P}_{\mathbf{V}, \Omega} \cong \mathscr{P}_{\mathbf{V}, \Omega^{\prime}}$.

(c) $\mathscr{F}$ defines an equivalence of categories $\mathscr{Q}_{\mathbf{V}, \Omega} \cong \mathscr{Q}_{\mathbf{V}, \Omega^{\prime}}$.

Using 5.4 and 3.7 (a) we see that it is enough to prove (a) in the special case where both $\mathbf{i}$, a have a single term, so that $\mathbf{V}$ is concentrated in a single degree. In this case, the maps $\delta, \delta^{\prime}$ in $5.2(\mathrm{a})$ are the identity, and $T$ of 5.2 is zero, so that $\mathscr{L}_{T}$ of 5.2 is 1 . Hence in this case, $\mathscr{F}$ is the identity functor and (a) follows (see 3.6) with $d=0$.

Now from (a) it follows that $\mathscr{F}(L) \in \mathscr{P}_{\mathbf{v}, \Omega^{\prime}}$ for any $L \in \mathscr{P}_{\mathbf{v}, \Omega}$, since $\mathscr{F}$ takes irreducible perverse sheaves to irreducible perverse sheaves (a general property of the Fourier-Deligne transform). Hence $\mathscr{F}$ defines a map $\mathscr{P}_{\mathbf{V}, \Omega} \rightarrow \mathscr{P}_{\mathbf{V}, \Omega^{\prime}}$. This map is injective by general properties of the FourierDeligne transform. Hence $\sharp \mathscr{P}_{\mathbf{v}, \Omega} \leq \sharp \mathscr{P}_{\mathbf{v}, \Omega^{\prime}}$. Reversing the roles of $\Omega, \Omega^{\prime}$, we obtain the reverse inequality; hence we have an equality and our injective map must be a bijection. (It is a map between finite sets, see 2.2(b).) Thus, (b) is proved. Clearly, (c) follows from (b).

\section{ANALYSIS OF A SINK}

6.1. In this section we shall assume that we are given an orientation $\Omega$ for our graph and a vertex $i \in I$.

Given $\mathbf{V} \in \mathscr{V}_{\nu}$ and an integer $r \geq 0$, we define $\mathbf{E}_{\mathbf{V}, \Omega, r}$ to be the set of all $x \in \mathbf{E}_{\mathbf{V}, \Omega}$ such that the sum (over all $h \in \Omega$ such that $h^{\prime \prime}=i$ ) of the images of $x_{h}: \mathbf{V}_{h^{\prime}} \rightarrow \mathbf{V}_{i}$ has codimension $r$ in $\mathbf{V}_{i}$. The $\mathbf{E}_{\mathbf{V}, \Omega, r}$ form a partition of $\mathbf{E}_{\mathbf{V}, \Omega}$ such that, for any $r \geq 0$, the union $\mathbf{E}_{\mathbf{V}, \Omega, \geq r}=\bigcup_{r^{\prime} \geq r} \mathbf{E}_{\mathbf{V}, \Omega, r^{\prime}}$ is closed in $\mathbf{E}_{\mathbf{V}, \Omega}$.

6.2. If $L \in \mathscr{P}_{\mathbf{v}, \Omega}$, we denote by $\sigma(L)$ the support of $L$ (a closed, irreducible, $G_{\mathbf{v}}$-invariant subvariety of $\left.\mathbf{E}_{\mathbf{v}, \Omega}\right)$. Let $t(L)$ be the unique integer $\geq 0$ such that $\sigma(L) \subset \mathbf{E}_{\mathbf{V}, \Omega, \geq t(L)}$ and $\sigma(L) \not \subset \mathbf{E}_{\mathbf{V}, \Omega, \geq t(L)+1}$. Then $\sigma_{0}(L)=\sigma(L) \cap \mathbf{E}_{\mathbf{V}, \Omega, t(L)}$ is an open dense $G_{\mathbf{v}}$-invariant subset of $\sigma(L)$.

We have $t(L) \leq \nu(i)$. 
6.3. For any $r \geq 0$, let $\nu_{r} \in \mathbf{N}^{I}$ be defined by $\nu_{r}(i)=r$ and $\nu_{r}(j)=0$ for all $j \in I, j \neq i$. We denote by $\mathbf{V}_{r}$ a vector space in $\mathscr{V}_{\nu_{r}}$. We denote by $1_{r}$ the complex 1 on $\mathbf{E}_{\mathbf{V}_{r}, \Omega}=\{0\}$.

Lemma 6.4. Assume that $i$ is a sink for $\Omega$, that is ,

$$
h \in \Omega \Rightarrow h^{\prime} \neq i \text {. }
$$

We fix a number $t$ such that $0 \leq t \leq \nu(i)$. Let $\mathbf{V} \in \mathscr{V}_{\nu}$ and let $\mathbf{W}$ be an $I$ graded subspace of $\mathbf{V}$ such that $\mathbf{W} \in \mathscr{V}_{\nu-\nu_{t}}$. Let $d=t(\nu(i)-t)$. Let $L \in \mathscr{P}_{\mathbf{V}, \Omega}$ and let $K \in \mathscr{P}_{\mathbf{w}, \Omega}$.

(a) Let $L \in \mathscr{P}_{\mathbf{v}, \Omega}$ be such that $t(L)=t$. Then $\operatorname{res}_{\mathbf{v} / \mathbf{w}, \mathbf{w}} L \in \mathscr{Q}_{\mathbf{w}, \Omega}$ is a direct sum of finitely many summands of the form $K^{\prime}\left[f^{\prime}\right]$ for various $K^{\prime} \in \mathscr{P}_{\mathbf{W}, \Omega}$ and various $f^{\prime} \in \mathbf{Z}$; exactly one of these summands satisfies $t\left(K^{\prime}\right)=0$ and $f^{\prime}=d$; the others satisfy $t\left(K^{\prime}\right)>0$.

(b) Let $K \in \mathscr{P}_{\mathbf{W}, \Omega}$ be such that $t(K)=0$. Then $1_{t} * K \in \mathscr{Q}_{\mathbf{V}, \Omega}$ is a direct sum of finitely many summands of the form $L^{\prime \prime}\left[f^{\prime \prime}\right]$ for various $L^{\prime \prime} \in \mathscr{P}_{\mathbf{V}, \Omega}$ and various $f^{\prime \prime} \in \mathbf{Z}$; exactly one of these summands satisfies $t\left(L^{\prime \prime}\right)=t$ and $f^{\prime \prime}=d$; the others satisfy $t\left(L^{\prime \prime}\right)>t$.

(c) Consider the sets $\left\{L \in \mathscr{P}_{\mathbf{v}, \Omega} \mid t(L)=t\right\}$ and $\left\{K \in \mathscr{P}_{\mathbf{w}, \Omega} \mid t(K)=0\right\}$. Associate to $L$ in the first set the $K$ in the second set such that some shift of $K$ is a summand of $\operatorname{res}_{\mathbf{V} / \mathbf{w}, \mathbf{w}} L$; associate to $K$ in the second set the $L$ in the first set such that some shift of $L$ is a summand of $1_{t} * K$. These give two bijections between our two sets, inverse to each other.

We first prove (b). We consider the commutative diagram

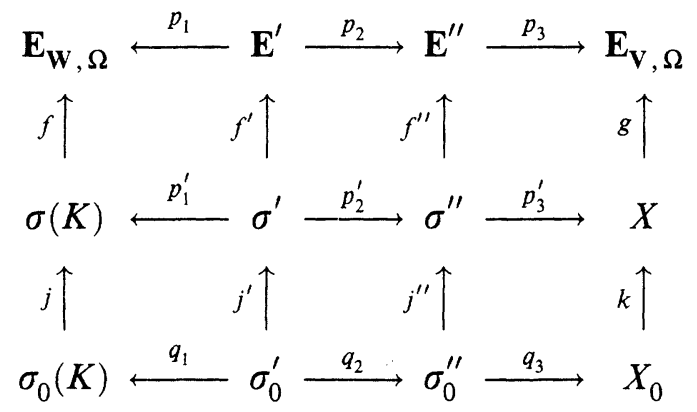

where the notations are as follows.

The first row is as in 3.1(a) (with $\mathbf{V}^{\prime \prime}=\mathbf{W}, \mathbf{V}^{\prime}=\mathbf{V} / \mathbf{W}$; note that $\mathbf{E}_{\mathbf{v} / \mathbf{w}, \Omega}=$ $0)$. We have $\sigma^{\prime}=p_{1}^{-1}(\sigma(K)), \sigma^{\prime \prime}=p_{2}\left(\sigma^{\prime}\right), X=p_{3}\left(\sigma^{\prime \prime}\right), \sigma_{0}^{\prime}=p_{1}^{-1}\left(\sigma_{0}(K)\right)$, $\sigma_{0}^{\prime \prime}=p_{2}\left(\sigma_{0}^{\prime}\right), X_{0}=X \cap \mathbf{E}_{\mathbf{v}, \Omega, t}$; the maps in the second and third row are induced by those in the first row and the vertical maps are the obvious inclusions. Let $K_{2} \in \mathscr{D}\left(\mathbf{E}^{\prime}\right), K_{3} \in \mathscr{D}\left(\mathbf{E}^{\prime \prime}\right)$ be semisimple complexes such that $p_{1}^{*}(K) \cong$ $p_{2}^{*}\left(K_{3}\right) \cong K_{2}$. By definition, $1_{t} * K=\left(p_{3}\right)_{!} K_{3} \in \mathscr{Q}_{\mathbf{V}, \Omega}$. We have $K=f_{!} K_{4}$ for a well-defined irreducible perverse $G_{\mathrm{w}}$-equivariant complex $K_{4} \in \mathscr{D}(\sigma(K))$. 
Hence there exist well-defined semisimple complexes $K_{5} \in \mathscr{D}\left(\sigma^{\prime}\right)$ and $K_{6} \in$ $\mathscr{D}\left(\sigma^{\prime \prime}\right)$ such that $\left(p_{1}^{\prime}\right)^{*}\left(K_{4}\right) \cong\left(p_{2}^{\prime}\right)^{*}\left(K_{6}\right) \cong K_{5}$. Let $K_{7}=\left(p_{3}^{\prime}\right)_{!} K_{6} \in \mathscr{D}(X)$. Now $f_{!}^{\prime} K_{5}$ and $f_{!}^{\prime \prime} K_{6}$ are semisimple since $f^{\prime}, f^{\prime \prime}$ are closed imbeddings. It follows that $f_{!}^{\prime} K_{5}=K_{2}$ and $f_{!}^{\prime \prime} K_{6}=K_{3}$. Clearly, $\left(p_{3}\right)_{!} K_{3}=g_{!} K_{7}$. In particular,

(d) the support of $\left(p_{3}\right)_{!} K_{3}$ is contained in $X$.

Let $K_{8}=j^{*} K_{4} \in \mathscr{D}\left(\sigma_{0}\right)$. This is an irreducible $G_{\mathrm{w}}$-equivariant complex since $j$ is the inclusion of an open dense set. Hence there exist well-defined semisimple complexes $K_{9} \in \mathscr{D}\left(\sigma_{0}^{\prime}\right)$ and $K_{10} \in \mathscr{D}\left(\sigma_{0}^{\prime \prime}\right)$ such that $q_{1}^{*}\left(K_{8}\right) \cong$ $q_{2}^{*}\left(K_{10}\right) \cong K_{9}$. Note that $K_{9}\left[d_{1}\right]$ and $K_{10}\left[d_{1}-d_{2}\right]$ are irreducible, perverse where $d_{1}, d_{2}$ are the dimensions of the fibres of $p_{1}, p_{2}$. Using the fact that $i$ is a sink, we see easily that $d_{1}-d_{2}=d$. Thus,

(e) $K_{10}[d]$ is an irreducible perverse sheaf.

Let $K_{11}=\left(q_{3}\right)_{!} K_{10} \in \mathscr{D}\left(X_{0}\right)$.

Using the fact that $j^{\prime}, j^{\prime \prime}$ are open imbeddings we see that $K_{9}=j^{\prime *} K_{5}$ and $K_{10}=j^{\prime \prime *} K_{6}$. Since the diagram $j^{\prime \prime}, p_{3}^{\prime}, q_{3}, k$ is cartesian, we have $k^{*} K_{7}=$ $K_{11}$. It is easy to check that $q_{3}$ is an isomorphism. Hence from (e) it follows that $K_{11}[d]$ is an irreducible perverse sheaf.

Summarizing, we see that the complex $\left(p_{3}\right)_{!} K_{3}[-d] \in D_{\mathbf{v}, \Omega}$ has support contained in $X$, and its restriction to the open set $X_{0}$ of $X$ is irreducible perverse; note that $X_{0} \subset \mathbf{E}_{\mathbf{v}, \Omega, t}$ and $X-X_{0} \subset \mathbf{E}_{\mathbf{v}, \Omega, \geq t+1}$. This clearly implies (b).

We now prove (a). Consider the commutative diagram

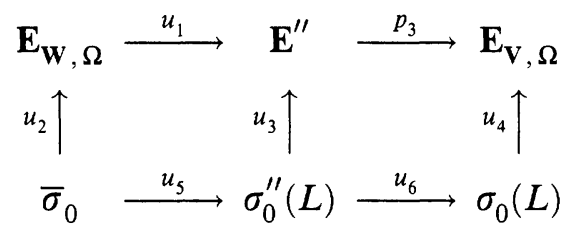

where the notation is as follows.

The map $u_{1}$ takes $y$ to $(x, \mathbf{W})$ where $x \in \mathbf{E}_{\mathbf{v}, \Omega}$ is uniquely determined by the requirements that $\mathbf{W}$ is $x$-stable and $y$ is induced by $x$. (This is well defined since $i$ is a sink.)

We have $\sigma_{0}^{\prime \prime}(L)=p_{3}^{-1}\left(\sigma_{0}(L)\right), \bar{\sigma}_{0}=u_{1}^{-1} \sigma_{0}^{\prime \prime}(L)$. The maps $u_{5}, u_{6}$ are induced by $u_{1}, p_{3}$; the vertical maps are the obvious inclusions.

The composition $p_{3} u_{1}$ identifies $\mathbf{E}_{\mathbf{W}, \Omega}$ with a subspace of $\mathbf{E}_{\mathbf{v}, \Omega}$, and it follows from the definitions that $\operatorname{res}_{\mathbf{v} / \mathbf{w}, \mathbf{w}} L=\left(p_{3} u_{1}\right)^{*} L \in \mathscr{Q}_{\mathbf{W}, \Omega}$. Clearly, $\bar{\sigma}_{0}$ is open in the support of $\left(p_{3} u_{1}\right)^{*} L$, is contained in $\mathbf{E}_{\mathbf{w}, \Omega, 0}$ and its complement in the support of $\left(p_{3} u_{1}\right)^{*} L$ is contained in $\mathbf{E}_{\mathbf{W}, \Omega, \geq 1}$. Hence, to prove (a) it is enough to prove that $u_{2}^{*}\left(p_{3} u_{1}\right)^{*} L[-d]$ is an irreducible perverse sheaf or, equivalently, that $u_{5}^{*} u_{6}^{*} u_{4}^{*} L[-d]$ is an irreducible perverse sheaf. Let $L_{1}=u_{4}^{*} L$. This is an irreducible perverse sheaf on $\sigma_{0}(L)$ since $\sigma_{0}$ is open dense in the support of $L$. Let $L_{2}=u_{6}^{*} L_{1}$. It is easy to see that $u_{6}$ is an isomorphism; hence $L_{2}$ is 
an irreducible perverse sheaf on $\sigma_{0}^{\prime \prime}(L)$. Consider the $G_{\mathbf{v}}$-equivariant fibration $\rho: \sigma_{0}^{\prime \prime}(L) \rightarrow \mathbf{G}$ where $\mathbf{G}$ is the variety consisting of all $I$-graded subspaces $V$ of $\mathbf{V}$ such that $\mathbf{V} / V \in \mathscr{V}_{\nu_{t}}$ and $\rho(x, V)=V$. Note that $u_{5}$ identifies $\bar{\sigma}_{0}$ with the fibre of $\rho$ at $\mathbf{W}$. Since $L_{2}$ is an equivariant perverse sheaf and $G_{\mathbf{V}}$ acts transitively on $\mathbf{G}$, it follows that $u_{5}^{*} L_{2}[-d]$ is an irreducible perverse sheaf on $\bar{\sigma}_{0}$ and (a) follows. (Note that $d=\operatorname{dim} \mathbf{G}$.)

Now (c) follows easily from the arguments in the proof of (a), (b). This completes the proof.

6.5. We no longer assume that $i$ is a sink for $\Omega$.

Let $L \in \mathscr{P}_{\mathbf{V}, \Omega}\left(\right.$ with $\left.\mathbf{V} \in \mathscr{V}_{\nu}\right)$.

For each $r$ such that $0 \leq r \leq \nu_{i}$ we shall denote by $\mathbf{W}_{r}$ some object of $\mathscr{V}_{\nu-\nu_{r}}$.

We associate to $L$ and $i$ an integer invariant $s(L)$ (or $s_{i}(L)$ ). By definition, $s(L)$ is the largest integer $r$ such that $0 \leq r \leq \nu(i)$ and such that

(a) there exists $L^{\prime} \in \mathscr{Q}_{\mathbf{W}_{r}, \Omega}$ such that some shift of $L$ is isomorphic to a direct summand of $1_{r} * L^{\prime} \in \mathscr{Q}_{\mathbf{V}, \Omega}$.

(This is well defined since (a) is satisfied with $r=0$.)

Proposition 6.6. Assume that we are in the setup of 6.5 and that $\mathbf{k}$ is as in 5.1.

(a) There exist complexes $L_{r^{\prime}}^{\prime}\left(s(L)<r^{\prime} \leq \nu(i)\right)$ and $L_{r^{\prime}}^{\prime \prime}\left(s(L) \leq r^{\prime} \leq \nu(i)\right)$ in $\mathscr{Q}_{\mathbf{W}_{r^{\prime}}, \Omega}$ such that

$$
L \oplus\left(\bigoplus_{r^{\prime}>s(L)} 1_{r^{\prime}} * L_{r^{\prime}}^{\prime}\right) \cong \bigoplus_{r^{\prime} \geq s(L)} 1_{r^{\prime}} * L_{r^{\prime}}^{\prime \prime}
$$

in $\mathscr{Q}_{\mathbf{V}, \Omega}$ and such that $L_{s(L)}^{\prime \prime}[f] \in \mathscr{P}_{\mathbf{w}_{s(L)}, \Omega}$ for some integer $f$.

(b) If $i$ is a sink for $\Omega$, then $s(L)=t(L)$.

We can find an orientation $\Omega^{\prime}$ for our graph such that $i$ is a sink for $\Omega^{\prime}$. Let $\mathscr{F}$ be as in 5.2.

Using 5.4, 5.6, we see that $s(\mathscr{F}(L))$ is defined and is equal to $s(L)$; we also see that the truth of (a) for $\mathscr{F}(L)$ implies the truth of (a) for $L$. Thus we see that it is enough to prove the proposition under the additional assumption that $i$ is a sink for $\Omega$.

From 6.4 we see that $6.5(\mathrm{a})$ is satisfied with $r=t(L)$; it follows that

(c) $s(L) \geq t(L)$.

Next we show that

(d) $s(L) \leq t(L)$.

Assume that 6.5(a) holds for some $r \leq \nu(i)$ and some $L^{\prime}$. Then it holds with some $L^{\prime} \in \mathscr{P}_{\mathbf{w}_{r}, \Omega}$; some shift of this $L^{\prime}$ is a direct summand of some $L_{\mathbf{i}, \mathbf{a}, \Omega}\left(\right.$ on $\mathbf{E}_{\mathbf{w}_{r}, \Omega}$ ) where $(\mathbf{i}, \mathbf{a}) \in S_{\nu-\nu_{r}}$. Hence 6.6(a) holds with $L^{\prime}=L_{\mathbf{i}, \mathbf{a}, \Omega}$.

Using 3.7(a) we see then that $1_{r} * L^{\prime}=L_{\mathbf{i}^{\prime}, \mathbf{a}^{\prime}, \Omega}$ for some $\left(\mathbf{i}^{\prime}, \mathbf{a}^{\prime}\right) \in S_{\nu}$ such that the first entry of $\mathbf{i}^{\prime}$ is $r$. 
Now the support of $L_{\mathbf{i}^{\prime}, \mathbf{a}^{\prime}, \Omega}$ is clearly contained in the set of all $x$ in $\mathbf{E}_{\mathbf{v}, \Omega}$ such that $x$ leaves stable some $I$-graded subspace $W$ of $\mathbf{V}$ with $\mathbf{V} / W \in \mathscr{\mathscr { V }}_{\nu_{r}}^{\Omega}$.

For such $x$ we have $x_{h}\left(\mathbf{V}_{h^{\prime}}\right) \subset W_{i}$ for all $h \in \Omega$ such that $h^{\prime \prime}=i$, since we then have $h^{\prime} \neq i$, and $x_{h}\left(\mathbf{V}_{h^{\prime}}\right)=x_{h}\left(W_{h^{\prime}}\right) \subset W_{i}$. This shows that the support of $L_{\mathbf{i}^{\prime}, \mathbf{a}^{\prime}, \Omega}$ is contained in $\mathbf{E}_{\mathbf{V}, \Omega, \geq r}$. Since some shift of $L$ is a direct summand of $L_{\mathbf{i}^{\prime}, \mathbf{a}^{\prime}, \Omega}$, it follows that the support of $L$ is contained in $\mathbf{E}_{\mathbf{V}, \Omega, \geq r}$. Thus $r \leq t(L)$ and (d) follows. Combining (c),(d) we see that (b) holds.

Next we prove (a); we may replace there $s(L)$ by $t(L)$ as we have just seen. Now (a) is trivial in the case where $t(L)=\nu(i)$ : in this case, we may take $L_{t(L)}^{\prime}=0, L_{t(L)}^{\prime \prime}=L$. Hence we may assume that $t(L)<\nu(i)$ and that (a) is already proved when $L$ is replaced by an $L^{\prime \prime}$ with $t\left(L^{\prime \prime}\right)>t(L)$.

Using 6.4 we see that there exists $K \in \mathscr{P}_{\mathbf{W}_{t(L)}, \Omega}$ such that $1_{t(L)} * K \in \mathscr{Q}_{\mathbf{V}, \Omega}$ is a direct sum of finitely many summands of the form $L^{\prime \prime}\left[f^{\prime \prime}\right]$ for various $L^{\prime \prime} \in \mathscr{P}_{\mathbf{V}, \Omega}$ and various $f^{\prime \prime} \in \mathbf{Z}$; exactly one of these summands satisfies $L^{\prime \prime}=L ;$ the others satisfy $t\left(L^{\prime \prime}\right)>t(L)$.

Applying the induction hypothesis to each $L^{\prime \prime} \neq L$ above we see that (a) holds for $L$. The proposition is proved.

\section{Multiplicative Generators}

7.1. In this section we fix an orientation $\Omega$ for our graph.

Lemma 7.2. Let $L \in \mathscr{P}_{\mathbf{V}, \Omega}$ with $\mathbf{V} \neq 0$. There exists some $i \in I$ such that $s_{i}(L)>0$. (See 6.5(a).)

We have $\mathbf{V} \in \mathscr{V}_{\nu}$. By the definition of $\mathscr{P}_{\mathbf{V}, \Omega}$, there exist $(\mathbf{i}, \mathbf{a}) \in S_{\nu}$ and $f \in \mathbf{Z}$ such that $L[f]$ is a direct summand of $L_{\mathbf{i}, \mathbf{a} ; \Omega}$ in $\mathscr{Q}_{\mathbf{V}, \Omega}$. As in 2.2, we see that we may assume that all coordinates of a are strictly positive; moreover, a is nonempty since $\mathbf{V} \neq 0$. Using now $3.7(\mathrm{a})$, we see that if we set $i=i_{1}$ (first entry of $\mathbf{i}$ ), then $s_{i}(L) \geq a_{1}>0$. The lemma is proved.

Proposition 7.3. Let $L \in \mathscr{P}_{\mathbf{V}, \Omega}$ with $\mathbf{V} \in \mathscr{V}_{\nu}$.

There exist $L_{1}, \ldots, L_{N}, L_{N+1}, \ldots, L_{N+M} \in \mathscr{Q}_{\mathbf{V}, \Omega}$ such that the following hold. $d$.

(a) Each $L_{j}$ is of the form $L_{\mathbf{i}, \mathbf{a} ; \Omega}[d]$ for some $(\mathbf{i}, \mathbf{a}) \in S_{\nu}$ and some integer

(b) $L \oplus\left(L_{1} \oplus \cdots \oplus L_{N}\right) \cong L_{N+1} \oplus \cdots \oplus L_{N+M}$ in $\mathscr{Q}_{\mathbf{V}, \Omega}$.

This is trivial for $\mathbf{V}=0$; hence we may assume that $\mathbf{V} \neq 0$ and that the result is already proved for graded vector spaces of total dimension strictly smaller than that of $\mathbf{V}$.

By general principles, we may assume that $\mathbf{k}$ is as in 5.1. By 7.2 we can find $i \in I$ so that $s_{i}(L)>0$. We apply 6.6(a) to $L$ and this $i$. The induction hypothesis is applicable to each $L_{r^{\prime}}^{\prime}, L_{r^{\prime}}^{\prime \prime}$ appearing there. The desired result follows, using $3.7(\mathrm{~b})$. 


\section{COMPATIBILITY OF MULTIPLICATION WITH RESTRICTION}

8.1. In this section we assume that we are given an orientation $\Omega$ for our graph.

We will need a variant of the construction of the product $*$ in 3.1. Assume that we are given six objects $\mathbf{T}, \mathbf{T}^{\prime}, \mathbf{T}^{\prime \prime}, \mathbf{W}, \mathbf{W}^{\prime}, \mathbf{W}^{\prime \prime}$ of $\mathscr{V}$ such that $\mathbf{T}$ is isomorphic to $\mathbf{T}^{\prime} \oplus \mathbf{T}^{\prime \prime}$ and $\mathbf{W}$ is isomorphic to $\mathbf{W}^{\prime} \oplus \mathbf{W}^{\prime \prime}$ (together with the grading). Consider the diagram

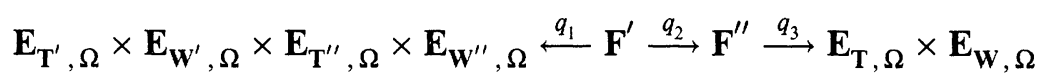

where the notations are as follows.

$\mathbf{F}^{\prime \prime}$ is the variety of all quadruples $(z, y, T, W)$ where $z \in \mathbf{E}_{\mathrm{T}, \Omega}, y \in$ $\mathbf{E}_{\mathbf{W}, \Omega}, T$ is a $z$-stable $I$-graded subspace of $\mathbf{T}$ such that $T$ is isomorphic to $\mathbf{T}^{\prime \prime}$ and $W$ is an $y$-stable $I$-graded subspace of $\mathbf{W}$ such that $W$ is isomorphic to $\mathbf{W}^{\prime \prime}$.

$\mathbf{F}^{\prime}$ is the variety of all sequences $(z, y, T, W, r, u, s, t)$ such that $(z, y, T, W) \in \mathbf{F}^{\prime \prime}$ and $r: \mathbf{T}^{\prime \prime} \cong T, u: \mathbf{T}^{\prime} \cong \mathbf{T} / T, s: \mathbf{W}^{\prime \prime} \cong W, t:$ $\mathbf{W}^{\prime} \cong \mathbf{W} / W$ are isomorphisms in $\mathscr{V}$.

We have $q_{1}(z, y, T, W, r, u, s, t)=\left(z^{\prime}, y^{\prime}, z^{\prime \prime}, y^{\prime \prime}\right)$ where $z_{h^{\prime}} r_{h^{\prime}}=r_{h^{\prime \prime}} z_{h^{\prime}}^{\prime \prime}$ : $\mathbf{T}_{h^{\prime}}^{\prime \prime} \rightarrow T_{h^{\prime \prime}}, z_{h} u_{h^{\prime}}=u_{h^{\prime \prime}} z_{h}^{\prime}: \mathbf{T}_{h^{\prime}}^{\prime} \rightarrow \mathbf{T}_{h^{\prime \prime}} / T_{h^{\prime \prime}}, y_{h^{\prime}} s_{h^{\prime}}=s_{h^{\prime \prime}} y_{h}^{\prime \prime}: \mathbf{W}_{h^{\prime}}^{\prime \prime} \rightarrow W_{h^{\prime \prime}}$, $y_{h} t_{h^{\prime}}=t_{h^{\prime \prime}} y_{h}^{\prime}: \mathbf{W}_{h^{\prime}}^{\prime} \rightarrow \mathbf{W}_{h^{\prime \prime}} / W_{h^{\prime \prime}}$ for all $h \in H ; q_{2}(z, y, T, W, r, u, s, t)=$ $(z, y, T, W), q_{3}(z, y, T, W)=(z, y)$.

Note that $q_{1}$ is smooth with connected fibres, $q_{2}$ is a $G_{\mathbf{T}^{\prime}} \times G_{\mathbf{W}^{\prime}} \times G_{\mathbf{T}^{\prime \prime}} \times G_{\mathbf{W}^{\prime \prime}}$ principal bundle, and $q_{3}$ is proper.

Now given $L^{\prime} \in \mathscr{Q}_{\mathbf{T}^{\prime}, \mathbf{w}^{\prime}, \Omega}$ and $L^{\prime \prime} \in \mathscr{Q}_{\mathbf{T}^{\prime \prime}, \mathbf{w}^{\prime \prime}, \Omega}$, we can form the external tensor product $L^{\prime} \otimes L^{\prime \prime}$ (a semisimple, $G_{\mathbf{T}^{\prime}} \times G_{\mathbf{W}^{\prime}} \times G_{\mathbf{T}^{\prime \prime}} \times G_{\mathbf{W}^{\prime \prime}}$-equivariant complex on $\left.\mathbf{E}_{\mathbf{T}^{\prime}, \Omega} \times \mathbf{E}_{\mathbf{W}^{\prime}, \Omega} \times \mathbf{E}_{\mathbf{T}^{\prime \prime}, \Omega} \times \mathbf{E}_{\mathbf{W}^{\prime \prime}, \Omega}\right)$. Then, just as in 3.1, there is a well-defined semisimple complex $\dot{L}$ on $\mathbf{F}^{\prime \prime}$ such that $q_{2}^{*}(\dot{L}) \cong q_{1}^{*}\left(L^{\prime} \otimes L^{\prime \prime}\right)$ in $\mathscr{D}\left(\mathbf{F}^{\prime}\right)$. By definition, $L^{\prime} * L^{\prime \prime}=\left(q_{3}\right)_{*}(\dot{L}) \in \mathscr{D}\left(\mathbf{E}_{\mathbf{T}, \Omega} \times \mathbf{E}_{\mathbf{W}, \Omega}\right)$. This is additive in $L^{\prime}$ and in $L^{\prime \prime}$; moreover, if $L^{\prime}$ is an external tensor product $L_{1} \otimes L_{2}$ (with $L_{1} \in \mathscr{Q}_{\mathbf{T}^{\prime}, \Omega}$ and $L_{2} \in \mathscr{Q}_{\mathbf{W}^{\prime}, \Omega}$ ) and $L^{\prime \prime}$ is an external tensor product $L_{3} \otimes L_{4}$ (with $L_{3} \in \mathscr{Q}_{\mathbf{T}^{\prime \prime}, \Omega}$ and $L_{4} \in \mathscr{Q}_{\mathbf{W}^{\prime \prime}, \Omega}$ ), then one can easily verify that $L^{\prime} * L^{\prime \prime}$ is isomorphic to the external tensor product $\left(L_{1} * L_{3}\right) \otimes\left(L_{2} * L_{4}\right)$, where $L_{1} * L_{3} \in \mathscr{Q}_{\mathbf{T}, \Omega}$ and $L_{2} * L_{4} \in \mathscr{Q}_{\mathbf{W}, \Omega}$ are defined as in 3.1 (with $\mathbf{V}, \mathbf{V}^{\prime}, \mathbf{V}^{\prime \prime}$ replaced by $\mathbf{T}, \mathbf{T}^{\prime}, \mathbf{T}^{\prime \prime}$ or by $\left.\mathbf{W}, \mathbf{W}^{\prime}, \mathbf{W}^{\prime \prime}\right)$. It follows that, in general, we have $L^{\prime} * L^{\prime \prime} \in \mathscr{Q}_{\mathrm{T}, \mathbf{W}, \Omega}$.

8.2. Assume now that we are given $\mathbf{V}, \mathbf{V}^{\prime}, \mathbf{V}^{\prime \prime} \in \mathscr{V}$ such that $\mathbf{V} \in \mathscr{V}_{\nu}, \mathbf{V}^{\prime} \in$ $\mathscr{V}_{\nu^{\prime}}, \mathbf{V}^{\prime \prime} \in \mathscr{V}_{\nu^{\prime \prime}}$, where $\nu, \nu^{\prime}, \nu^{\prime \prime} \in \mathbf{N}^{I}$ satisfy $\nu=\nu^{\prime}+\nu^{\prime \prime}$ (as in 3.1). At the same time we are given (as in 4.1) an $I$-graded subspace $\mathbf{W}$ of $\mathbf{V}$ with $\mathbf{W} \in \mathscr{V}_{\omega}$. Let $\mathbf{T}=\mathbf{V} / \mathbf{W}$. Then $\mathbf{T} \in \mathscr{V}_{\tau}$ where $\nu=\tau+\omega$.

Let $L^{\prime} \in \mathscr{Q}_{\mathbf{V}^{\prime}, \Omega}$ and let $L^{\prime \prime} \in \mathscr{Q}_{\mathbf{V}^{\prime \prime}, \Omega}$. Then $L^{\prime} * L^{\prime \prime} \in \mathscr{Q}_{\mathbf{V}, \Omega}$ is defined as in 3.1, 3.2, and $\left.\operatorname{res}_{\mathbf{T}, \mathbf{W}}\left(L^{\prime} * L^{\prime \prime}\right)\right) \in \mathscr{Q}_{\mathbf{T}, \mathbf{W}, \Omega}$ is defined as in 4.1, 4.2. 
8.3. Let $\mathcal{N}$ be the set of all ordered quadruples $\kappa=\left(\alpha^{\prime}, \beta^{\prime}, \alpha^{\prime \prime}, \beta^{\prime \prime}\right)$ of elements of $\mathbf{N}^{I}$ such that $\nu^{\prime}=\alpha^{\prime}+\beta^{\prime}, \nu^{\prime \prime}=\alpha^{\prime \prime}+\beta^{\prime \prime}, \tau=\alpha^{\prime}+\alpha^{\prime \prime}, \omega=\beta^{\prime}+\beta^{\prime \prime}$. For each $\kappa=\left(\alpha^{\prime}, \beta^{\prime}, \alpha^{\prime \prime}, \beta^{\prime \prime}\right) \in \mathscr{N}$ we choose a graded subspace $\mathbf{W}^{\prime}(\kappa)$ of $\mathbf{V}^{\prime}$ and a graded subspace $\mathbf{W}^{\prime \prime}(\kappa)$ of $\mathbf{V}^{\prime \prime}$ such that $\mathbf{W}^{\prime}(\kappa) \in \mathscr{V}_{\beta^{\prime}}$ and $\mathbf{W}^{\prime \prime}(\kappa) \in \mathscr{V}_{\beta^{\prime \prime}}$. Let $\mathbf{T}^{\prime}(\kappa)=\mathbf{V}^{\prime} / \mathbf{W}^{\prime}(\kappa), \mathbf{T}^{\prime \prime}(\kappa)=\mathbf{V}^{\prime \prime} / \mathbf{W}^{\prime \prime}(\kappa) ;$ then $\mathbf{T}^{\prime}(\kappa) \in \mathscr{V}_{\alpha^{\prime}}$ and $\mathbf{T}^{\prime \prime}(\kappa) \in \mathscr{\mathscr { V }}_{\alpha^{\prime \prime}}$.

Applying the construction of 4.1 to $\mathbf{V}^{\prime}, \mathbf{W}^{\prime}(\kappa), \mathbf{T}^{\prime}(\kappa), L^{\prime}$ (resp. to $\mathbf{V}^{\prime \prime}$, $\left.\mathbf{W}^{\prime \prime}(\kappa), \mathbf{T}^{\prime \prime}(\kappa), L^{\prime \prime}\right)$ instead of $\mathbf{V}, \mathbf{W}, \mathbf{T}, K$, we obtain a complex $L_{\kappa}^{\prime}=$ $\operatorname{res}_{\mathbf{T}^{\prime}(\kappa), \mathbf{w}^{\prime}(\kappa)} L^{\prime} \in \mathscr{Q}_{\mathbf{T}^{\prime}(\kappa), \mathbf{w}^{\prime}(\kappa), \Omega}\left(\right.$ resp. $\left.L_{\kappa}^{\prime \prime}=\operatorname{res}_{\mathbf{T}^{\prime \prime}(\kappa), \mathbf{w}^{\prime \prime}(\kappa)} L^{\prime \prime} \in \mathscr{Q}_{\mathbf{T}^{\prime \prime}(\kappa), \mathbf{w}^{\prime \prime}(\kappa), \Omega}\right)$ instead of $\operatorname{res}_{\mathbf{T}, \mathbf{W}} L \in \mathscr{Q}_{\mathbf{T}, \mathbf{w}, \Omega}$.

Next, from $L_{\kappa}^{\prime}$ and $L_{\kappa}^{\prime \prime}$ we can construct a complex $L_{\kappa}^{\prime} * L_{\kappa}^{\prime \prime} \in \mathscr{Q}_{\mathbf{T}, \mathbf{w}, \Omega}$ by the construction in 8.1 applied to $\mathbf{T}, \mathbf{T}^{\prime}=\mathbf{T}^{\prime}(\kappa), \mathbf{T}^{\prime \prime}=\mathbf{T}^{\prime \prime}(\kappa), \mathbf{W}, \mathbf{W}^{\prime}=$ $\mathbf{W}^{\prime}(\kappa), \mathbf{W}^{\prime \prime}=\mathbf{W}^{\prime \prime}(\kappa)$. We can now state the following result.

Proposition 8.4. Assume that $\mathbf{k}$ is as in 5.1. With the notations in 8.2, 8.3, we have

$$
\operatorname{res}_{\mathbf{T}, \mathbf{w}}\left(L^{\prime} * L^{\prime \prime}\right) \cong \bigoplus_{\kappa \in \mathscr{N}} L_{\kappa}^{\prime} * L_{\kappa}^{\prime \prime}[-2 g(\kappa)]
$$

in $\mathscr{Q}_{\mathbf{T}, \mathbf{W}, \Omega}$, where

$$
g(\kappa)=\sum_{i, j \in I} \alpha^{\prime}(i) \beta^{\prime \prime}(j) \sharp\left\{h \in \Omega \mid h^{\prime}=i, h^{\prime \prime}=j\right\}+\sum_{i \in I} \alpha^{\prime \prime}(i) \beta^{\prime}(i) .
$$

Let $K^{\prime}, M^{\prime} \in \mathscr{Q}_{\mathbf{V}^{\prime}, \Omega}$ and $K^{\prime \prime}, M^{\prime \prime} \in \mathscr{Q}_{\mathbf{V}^{\prime \prime}, \Omega}$ be such that $L^{\prime} \oplus K^{\prime} \cong M^{\prime}$ and $L^{\prime \prime} \oplus K^{\prime \prime} \cong M^{\prime \prime}$. Clearly, if the proposition is true for $\left(K^{\prime}, K^{\prime \prime}\right)$ and for $\left(M^{\prime}, M^{\prime \prime}\right)$ (instead of $\left(L^{\prime}, L^{\prime \prime}\right)$ ) then it is automatically true for $\left(L^{\prime}, L^{\prime \prime}\right)$.

By 7.3, we can find $K^{\prime}, M^{\prime}, K^{\prime \prime}, M^{\prime \prime}$ as above, which are direct sums of shifts of complexes of the form $L_{\mathrm{i}, \mathbf{a} ; \Omega}$. We see therefore that it is enough to prove the proposition under the additional assumption that $L, L^{\prime}$ are direct sums of shifts of complexes of the form $L_{\mathbf{i}, \mathbf{a} ; \Omega}$.

It follows immediately that it is enough to prove the proposition in the special case where $L^{\prime}=L_{\mathbf{i}^{\prime}, \mathbf{a}^{\prime} ; \Omega}$ and $L^{\prime \prime}=L_{\mathbf{i}^{\prime \prime}, \mathbf{a}^{\prime \prime} ; \Omega}\left(\right.$ with $\left(\mathbf{i}^{\prime}, \mathbf{a}^{\prime}\right) \in S_{\nu^{\prime}}$ and $\left(\mathbf{i}^{\prime \prime}, \mathbf{a}^{\prime \prime}\right) \in$ $\left.S_{\nu^{\prime \prime}}\right)$.

Define $(\mathbf{i}, \mathbf{a}) \in S_{\nu}$ as in 3.2 ; then $L^{\prime} * L^{\prime \prime}=L_{\mathbf{i}, \mathbf{a} ; \Omega}$ by $3.2(\mathbf{b})$ and, by 4.2 , we have

$$
\operatorname{res}_{\mathbf{T}, \mathbf{w}}\left(L^{\prime} * L^{\prime \prime}\right) \cong \bigoplus_{\mathbf{c}^{\prime}, \mathbf{c}^{\prime \prime}} L_{\mathbf{i}, \mathbf{c}^{\prime} ; \Omega} \otimes L_{\mathbf{i}, \mathbf{c}^{\prime \prime} ; \Omega}\left[-2 M\left(\mathbf{c}^{\prime}, \mathbf{c}^{\prime \prime}\right)\right]
$$

where the summation is over the pairs of sequences $\mathbf{c}^{\prime}, \mathbf{c}^{\prime \prime}$ such that $\left(\mathbf{i}, \mathbf{c}^{\prime}\right) \in S_{\tau}$, $\left(\mathbf{i}, \mathbf{c}^{\prime \prime}\right) \in S_{\omega}$ and $\mathbf{c}^{\prime}+\mathbf{c}^{\prime \prime}=\mathbf{a}$. Here,

$$
M\left(\mathbf{c}^{\prime}, \mathbf{c}^{\prime \prime}\right)=\sum_{l^{\prime} \leq l} \sharp\left\{h \in \Omega \mid h^{\prime}=i_{l^{\prime}}, h^{\prime \prime}=i_{l}\right\} c_{l^{\prime}}^{\prime} c_{l}^{\prime \prime}+\sum_{l<l^{\prime}: i_{l^{\prime}}=i_{l}} c_{l^{\prime}}^{\prime} c_{l}^{\prime \prime} .
$$


Let us now fix $\kappa \in \mathscr{N}$ as in 8.3. By 4.2,

$$
L_{\kappa}^{\prime} \cong \underset{\mathbf{d}^{\prime}, \mathbf{e}^{\prime}}{\bigoplus} L_{\mathbf{i}^{\prime}, \mathbf{d}^{\prime \prime} ; \Omega} \otimes L_{\mathbf{i}^{\prime}, \mathbf{e}^{\prime \prime} ; \Omega}\left[-2 M\left(\mathbf{d}^{\prime}, \mathbf{e}^{\prime}\right)\right]
$$

where the summation is over the pairs of sequences $\mathbf{d}^{\prime}, \mathbf{e}^{\prime}$ such that $\left(\mathbf{i}^{\prime}, \mathbf{d}^{\prime}\right) \in$ $S_{\alpha^{\prime}},\left(\mathbf{i}^{\prime}, \mathbf{e}^{\prime}\right) \in S_{\beta^{\prime}}$ and $\mathbf{d}^{\prime}+\mathbf{e}^{\prime}=\mathbf{a}^{\prime}$. Similarly,

$$
L_{\kappa}^{\prime \prime} \cong \bigoplus_{\mathbf{d}^{\prime \prime}, \mathbf{e}^{\prime \prime}} L_{\mathrm{i}^{\prime \prime}, \mathbf{d}^{\prime \prime} ; \Omega} \otimes L_{\mathrm{i}^{\prime \prime}, \mathbf{e}^{\prime \prime} ; \Omega}\left[-2 M\left(\mathbf{d}^{\prime \prime}, \mathbf{e}^{\prime \prime}\right)\right]
$$

where the summation is over the pairs of sequences $d^{\prime \prime}, e^{\prime \prime}$ such that $\left(i^{\prime \prime}, d^{\prime \prime}\right) \in$ $S_{\alpha^{\prime \prime}},\left(\mathbf{i}^{\prime \prime}, \mathbf{e}^{\prime \prime}\right) \in S_{\beta^{\prime \prime}}$ and $\mathbf{d}^{\prime \prime}+\mathbf{e}^{\prime \prime}=\mathbf{a}^{\prime \prime}$.

Using now 3.2 (b) and the results in 8.1 , we see that

(b) $\quad L_{\kappa}^{\prime} * L_{\kappa}^{\prime \prime} \cong \bigoplus L_{\mathbf{i}^{\prime} \mathbf{i}^{\prime \prime}, \mathbf{d}^{\prime} \mathbf{d}^{\prime \prime} ; \Omega} \otimes L_{\mathbf{i}^{\prime} \mathbf{i}^{\prime \prime}, \mathbf{e}^{\prime} \mathbf{e}^{\prime \prime} ; \Omega}\left[-2 M\left(\mathbf{d}^{\prime}, \mathbf{e}^{\prime}\right)-2 M\left(\mathbf{d}^{\prime \prime}, \mathbf{e}^{\prime \prime}\right)\right]$

where the summation is taken over the quadruples of sequences $d^{\prime}, d^{\prime \prime}, e^{\prime}, e^{\prime \prime}$ such that $\left(\mathbf{i}^{\prime}, \mathbf{d}^{\prime}\right) \in S_{\alpha^{\prime}},\left(\mathbf{i}^{\prime \prime}, \mathbf{d}^{\prime \prime}\right) \in S_{\alpha^{\prime \prime}},\left(\mathbf{i}^{\prime}, \mathbf{e}^{\prime}\right) \in S_{\beta^{\prime}},\left(\mathbf{i}^{\prime \prime}, \mathbf{e}^{\prime \prime}\right) \in S_{\beta^{\prime \prime}}, \mathbf{d}^{\prime}+\mathbf{e}^{\prime}=$ $\mathbf{a}^{\prime}$, and $\mathbf{d}^{\prime \prime}+\mathbf{e}^{\prime \prime}=\mathbf{a}^{\prime \prime}$. (Here $\mathbf{i}^{\prime} \mathbf{i}^{\prime \prime}$ is the sequence obtained from the sequence $\mathbf{i}^{\prime}$ followed by $\mathbf{i}^{\prime \prime}$ and $\mathbf{d}^{\prime} \mathbf{d}^{\prime \prime}, \mathbf{e}^{\prime} \mathbf{e}^{\prime \prime}$ have a similar meaning.) We note the identity

$$
g(\kappa)=M\left(\mathbf{d}^{\prime} \mathbf{d}^{\prime \prime}, \mathbf{e}^{\prime} \mathbf{e}^{\prime \prime}\right)-M\left(\mathbf{d}^{\prime}, \mathbf{e}^{\prime}\right)-M\left(\mathbf{d}^{\prime \prime}, \mathbf{e}^{\prime \prime}\right),
$$

which is easily verified. Using this identity together with (a) and (b), we obtain the desired result.

\section{RANK 2}

9.1. In this section we shall assume that $I$ has exactly two elements: $i$ and $j$. Let $N$ be the number of edges joining $i, j$; thus, $H$ has exactly $2 N$ elements.

Let $\mathbf{V} \in \mathscr{V}$ be such that $\operatorname{dim} V_{i}=1, \operatorname{dim} V_{j}=N+1$. Let $\Omega_{0}=\{h \in$ $\left.H \mid h^{\prime \prime}=j\right\}$.

9.2. We now fix an orientation $\Omega$ for our graph (not necessarily $\Omega_{0}$ ).

Let $\Omega^{\prime}=\left\{h \in \Omega \mid h^{\prime}=i\right\}, \Omega^{\prime \prime}=\left\{h \in \Omega \mid h^{\prime \prime}=i\right\}$. We denote by $a^{\prime}, a^{\prime \prime}$ the number of elements of $\Omega^{\prime}, \Omega^{\prime \prime}$ respectively.

The set $\mathscr{N}$ of nilpotent elements in $\mathbf{E}_{\mathbf{v}, \Omega}$ is the set of all $x \in \mathbf{E}_{\mathbf{v}, \Omega}$ such that $x_{h_{1}} x_{h_{2}}=0$ for all $h_{1} \in \Omega^{\prime \prime}, h_{2} \in \Omega^{\prime}$ or, equivalently, such that the sum of images of the maps $x_{h}\left(h \in \Omega^{\prime}\right)$ is contained in the intersection of the kernels of the maps $x_{h} \quad\left(h \in \mathbf{\Omega}^{\prime \prime}\right)$.

We define a stratification $\mathscr{N}=\bigcup_{p^{\prime} \geq p^{\prime \prime}} \mathscr{N}_{p^{\prime}, p^{\prime \prime}}$ as follows: $\mathscr{N}_{p^{\prime}, p^{\prime \prime}}$ is the set of all $x \in \mathscr{N}$ such that the sum of images of the maps $x_{h}\left(h \in \Omega^{\prime}\right)$ has codimension $p^{\prime}$ and the intersection of the kernels of the maps $x_{h}\left(h \in \Omega^{\prime \prime}\right)$ has codimension $p^{\prime \prime}$ in $\mathbf{V}_{j}$.

Clearly,

(a) $\mathscr{N}_{p^{\prime}, p^{\prime \prime}}$ is nonempty if and only if $N+1-p^{\prime} \leq a^{\prime}, p^{\prime \prime} \leq a^{\prime \prime}, p^{\prime} \geq p^{\prime \prime}$; if these conditions are satisfied, then $\mathscr{N}_{p^{\prime}, p^{\prime \prime}}$ is smooth, irreducible, of dimension $a^{\prime}\left(N+1-p^{\prime}\right)+a^{\prime \prime} p^{\prime \prime}+p^{\prime \prime}\left(p^{\prime}-p^{\prime \prime}\right)+p^{\prime}\left(N+1-p^{\prime}\right)$. 
9.3. For any $p \in[0, N+1]$ let $\widetilde{S}_{p}$ be the variety of all pairs $(x, W)$ where $x \in \mathbf{E}_{\mathbf{v}, \Omega}, W$ is a codimension $p$ subspace of $\mathbf{V}_{j}$, and $x_{h}\left(\mathbf{V}_{i}\right) \subset W\left(h \in \Omega^{\prime}\right)$, $x_{h} \mid W=0\left(h \in \Omega^{\prime \prime}\right)$. Let $S_{p} \subset \mathbf{E}_{\mathbf{V}, \Omega}$ be the image of the first projection $\widetilde{S}_{p} \rightarrow \mathbf{E}_{\mathbf{V}, \Omega}$. Then the first projection defines a proper map $\pi_{p}: \widetilde{S}_{p} \rightarrow S_{p}$.

It is easy to see that $S_{a^{\prime \prime}}=S_{a^{\prime \prime}+1}$, but apart from this, the subvarieties $S_{p}$ are distinct.

Proposition 9.4. Let $I_{p}(p \in[1, N+1])$ be the simple perverse sheaf on $\mathbf{E}_{\mathbf{V}, \Omega}$ defined by the subvariety $S_{p}$ and the local system 1 on its smooth part. Let $I_{p}^{\prime}$ $(p \in[0, N+1])$ be the complex of sheaves $\left(\pi_{p}\right)_{!}(1)\left[\operatorname{dim} S_{p}\right]$ extended by zero on the complement of $S_{p}$ in $\mathbf{E}_{\mathbf{V}, \Omega}$. We have

(a) $I_{0}^{\prime}=I_{0}, I_{N+1}^{\prime}=I_{N+1}$,

(b) $I_{p}^{\prime}=I_{p} \oplus I_{p-1}$ if $1 \leq p \leq a^{\prime \prime}, I_{p}^{\prime}=I_{p} \oplus I_{p+1}$ if $a^{\prime \prime}+1 \leq p \leq N$.

(c) $I_{a^{\prime \prime}}=I_{a^{\prime \prime}+1}$.

Note that

(d) $S_{p}$ is the union of the strata $\mathscr{N}_{p^{\prime}, p^{\prime \prime}}$ such that $p^{\prime} \geq p \geq p^{\prime \prime}, N+1-p^{\prime} \leq$ $a^{\prime}, p^{\prime \prime} \leq a^{\prime \prime}$.

One of these strata will be open, namely the one with minimal $p^{\prime}$ and maximal $p^{\prime \prime}$. Thus, if $p>a^{\prime \prime}$, then $p \geq N+1-a^{\prime}=a^{\prime \prime}+1$ and the open stratum is $\mathscr{N}_{p, a^{\prime \prime}}$; if $p \leq a^{\prime \prime}$, then $p<N+1-a^{\prime}=a^{\prime \prime}+1$ and the open stratum is $\mathscr{N}_{a^{\prime \prime}+1, p}$. In particular, $S_{a^{\prime \prime}}=S_{a^{\prime \prime}+1}$ since they have the same open dense stratum, $\mathscr{N}_{a^{\prime \prime}+1, a^{\prime \prime}}$. Using 9.2(a), we see that the dimension of $S_{p}$ is equal to $\left(p+a^{\prime}\right)(N+1-p)+a^{\prime \prime} p$.

We now consider the fibre $F$ of $\pi_{p}: \widetilde{S}_{p} \rightarrow S_{p}$ at a point of $\mathscr{N}_{p^{\prime}, p^{\prime \prime}}$ (as in (d)). We compute $\beta\left(p^{\prime}, p^{\prime \prime}\right)=\operatorname{dim} S_{p}-\operatorname{dim} \mathscr{N}_{p^{\prime}, p^{\prime \prime}}-2 \operatorname{dim} F$. Note that $\operatorname{dim} F=\left(p^{\prime}-p\right)\left(p-p^{\prime \prime}\right)$. It follows that $\beta\left(p^{\prime}, p^{\prime \prime}\right)=\left(p^{\prime}-a^{\prime \prime}-1\right)\left(p^{\prime}-p\right)+$ $\left(a^{\prime \prime}-p^{\prime \prime}\right)\left(p-p^{\prime \prime}\right)-\left(p^{\prime}-p\right)\left(p-p^{\prime \prime}\right)$. We can write $\beta\left(p^{\prime}, p^{\prime \prime}\right)$ in two different ways:

(e) $\beta\left(p^{\prime}, p^{\prime \prime}\right)=\left(p^{\prime}-a^{\prime \prime}-1\right)\left(p^{\prime}-p\right)+\lambda\left(p-p^{\prime \prime}\right)$ and

(f) $\beta\left(p^{\prime}, p^{\prime \prime}\right)=\left(a^{\prime \prime}-p^{\prime \prime}\right)\left(p-p^{\prime \prime}\right)+(-\lambda-1)\left(p^{\prime}-p\right)$

where $\lambda=a^{\prime \prime}+p-p^{\prime}-p^{\prime \prime}$.

We have $p^{\prime}-a^{\prime \prime}-1 \geq 0, p^{\prime} \geq p \geq p^{\prime \prime}$; moreover, clearly, either $\lambda$ or $-\lambda-1$ is $\geq 0$. Using one of the expressions (e) or (f) for $\beta\left(p^{\prime}, p^{\prime \prime}\right)$ we see that $\beta\left(p^{\prime}, p^{\prime \prime}\right) \geq 0$.

Assume now that $\beta\left(p^{\prime}, p^{\prime \prime}\right)=0$.

If $\lambda \leq-2$, we see from (f) that $p^{\prime}=p$ and either $p^{\prime \prime}=p$ or $p^{\prime \prime}=a^{\prime \prime}$. The alternative $p^{\prime \prime}=a^{\prime \prime}$ cannot occur since it would imply $\lambda=0$; the alternative $p^{\prime \prime}=p$ cannot occur either since it would imply $p^{\prime}=p^{\prime \prime}$ contradicting $p^{\prime}>$ $a^{\prime \prime} \geq a^{\prime \prime}$. 
If $\lambda>0$, we see from (e) that $p=p^{\prime \prime}$ and either $p^{\prime}=p$ or $p^{\prime}=a^{\prime \prime}+$ 1. The alternative $p^{\prime}=a^{\prime \prime}+1$ cannot occur since it would imply that $\lambda=$ -1 ; the alternative $p^{\prime}=p$ cannot occur either since it would imply $p^{\prime}=p^{\prime \prime}$ contradicting $p^{\prime}>a^{\prime \prime} \geq a^{\prime \prime}$.

If $\lambda=0$, we see from (e) that either $p^{\prime}=p$ and $p^{\prime \prime}=a^{\prime \prime}$ or $p^{\prime}=a^{\prime \prime}+1$ and $p^{\prime \prime}=p-1$; in the first case, we have $p \geq a^{\prime \prime}+1$, while in the second case we have $p \leq a^{\prime \prime}+1$.

If $\lambda=-1$, we see from (f) that either $p=p^{\prime \prime}$ and $p^{\prime}=a^{\prime \prime}+1$ or $p^{\prime \prime}=a^{\prime \prime}$ and $p^{\prime}=p+1$; in the first case, we have $p \leq a^{\prime \prime}$, while in the second case we have $p \geq a^{\prime \prime}$.

The inequality $\beta\left(p^{\prime}, p^{\prime \prime}\right) \geq 0$ shows that the proper map $\pi_{p}: \widetilde{S}_{p} \rightarrow S_{p}$ is semismall in the sense of Goresky and MacPherson. (Note that $\widetilde{S}_{p}$ is smooth.) Hence $I_{p}^{\prime}$ is a direct sum of finitely many simple perverse sheaves on $\mathbf{E}_{\mathbf{v}, \Omega}$. One of these is necessarily $I_{p}$, which appears with multiplicity one. Now the cohomology sheaves of $\left(\pi_{p}\right)_{!}(1)$ are constant on each stratum $\mathscr{N}_{p^{\prime}, p^{\prime \prime}}$ since $\pi_{p}$ restricted to a stratum is a Grassmannian bundle. It follows that any summand of $I_{p}^{\prime}$ other than $I_{p}$ must be a simple perverse sheaf defined by the closure of one of the strata with constant coefficients. The strata that contribute are determined by the equation $\beta\left(p^{\prime}, p^{\prime \prime}\right)=0$. As we have seen earlier, this equation has at most one solution other than the open stratum, and the closure of that stratum is either $S_{p+1}$ or $S_{p-1}$. This solution, if it exists, gives a summand that appears with multiplicity one in $I_{p}^{\prime}$ since the fibres of $\pi_{p}$ are irreducible. The proposition follows.

Corollary 9.5. In $\mathscr{D}\left(\mathbf{E}_{\mathbf{v}, \Omega}\right)$ we have

$$
\bigoplus_{p \text { even }} I_{p}^{\prime} \cong \bigoplus_{p \text { odd }} I_{p}^{\prime}
$$

where $p$ is subject to $0 \leq p \leq N+1$ in both sums.

9.6. The set $\mathscr{P}_{\mathrm{V}, \Omega}$ consists in our case of $I_{p}(p \in[1, N+1])$; this follows from the definitions and from $2.2(\mathrm{a}), 2.4(\mathrm{~b})$.

\section{Definition of the canonical basis B of $\mathbf{U}^{-}$}

10.1. We shall again fix an orientation $\Omega$ for our graph. Let $\mathscr{K}_{\mathbf{V}, \Omega}$ be the abelian group with one generator $(L)$ for each isomorphism class of objects of $\mathscr{Q}_{\mathbf{v}, \Omega}$ and with relations $(L)+\left(L^{\prime}\right)=\left(L^{\prime \prime}\right)$ whenever $L^{\prime \prime}$ is isomorphic to $L \oplus L^{\prime}$.

We regard $\mathscr{K}_{\mathbf{v}, \Omega}$ as a module over $\mathscr{A}=\mathbf{Z}\left[v, v^{-1}\right]$ ( $v$ is an indeterminate) by defining $v(L)=(L[1]), v^{-1}(L)=(L[-1])$. It is clearly a free $\mathscr{A}$-module with basis $(L)$ where $L$ runs over $\mathscr{P}_{\mathbf{V}, \Omega}$.

From 2.3 we see that, given $\mathbf{V}, \tilde{\mathbf{V}}$ in $\mathscr{V}_{\nu}$, there is a canonical isomorphism $\mathscr{K}_{\mathbf{V}, \Omega} \cong \mathscr{K}_{\mathbf{V}, \Omega}$. Hence we obtain a $\mathscr{A}$-module $\mathscr{K}_{\nu, \Omega}$ provided with natural isomorphisms $\mathscr{K}_{\nu, \Omega} \cong \mathscr{K}_{\mathbf{V}, \Omega}$, for any $\mathbf{V} \in \mathscr{V}_{\nu}$. We may regard $L_{\mathbf{i}, \mathbf{a} ; \Omega}$ as 
elements of $\mathscr{K}_{\nu, \Omega}$ for any $(\mathbf{i}, \mathbf{a}) \in S_{\nu}$. On the other hand, $\mathscr{P}_{\mathbf{v}, \Omega}$ gives rise to a canonical basis of $\mathscr{K}_{\nu, \Omega}$.

10.2. The operation $*$ in 3.1 defines a $\mathscr{A}$-bilinear map (denoted $\left(a^{\prime}, a^{\prime \prime}\right) \rightarrow$ $\left.a^{\prime} * a^{\prime \prime}\right): \mathscr{K}_{\nu^{\prime}, \Omega} \times \mathscr{K}_{\nu^{\prime \prime}, \Omega} \rightarrow \mathscr{K}_{\nu, \Omega}$, for any $\nu^{\prime}, \nu^{\prime \prime}, \nu \in \mathbf{N}^{I}$ such that $\nu=\nu^{\prime}+\nu^{\prime \prime}$. This has the associativity property $\left(a^{\prime} * a^{\prime \prime}\right) * a^{\prime \prime \prime}=a^{\prime} *\left(a^{\prime \prime} * a^{\prime \prime \prime}\right)$ for any $a^{\prime} \in \mathscr{K}_{\nu^{\prime}}$, $a^{\prime \prime} \in \mathscr{K}_{\nu^{\prime \prime}}, a^{\prime \prime \prime} \in \mathscr{K}_{\nu^{\prime \prime \prime}}$. (See 3.4.)

We now define a new $\mathscr{A}$-bilinear map $\mathscr{K}_{\nu^{\prime}, \Omega} \times \mathscr{K}_{\nu^{\prime \prime}, \Omega} \rightarrow \mathscr{K}_{\nu, \Omega}$ (for $\nu=$ $\left.\nu^{\prime}+\nu^{\prime \prime}\right)$ by $\left(a^{\prime}, a^{\prime \prime}\right) \rightarrow a^{\prime} \circ a^{\prime \prime}=v^{m_{\Omega}\left(\nu^{\prime}, \nu^{\prime \prime}\right)} a^{\prime} * a^{\prime \prime}$ where

$$
m_{\Omega}\left(\nu^{\prime}, \nu^{\prime \prime}\right)=\sum_{h \in \Omega} \nu^{\prime}\left(h^{\prime}\right) \nu^{\prime \prime}\left(h^{\prime \prime}\right)+\sum_{i} \nu^{\prime}(i) \nu^{\prime \prime}(i) .
$$

This again have the associativity property $\left(a^{\prime} \circ a^{\prime \prime}\right) \circ a^{\prime \prime \prime}=a^{\prime} \circ\left(a^{\prime \prime} \circ a^{\prime \prime \prime}\right)$ for any $a^{\prime} \in \mathscr{K}_{\nu^{\prime}, \Omega}, a^{\prime \prime} \in \mathscr{K}_{\nu^{\prime \prime}, \Omega}, a^{\prime \prime \prime} \in \mathscr{K}_{\nu^{\prime \prime \prime}, \Omega}$. (This follows from the identity $m_{\Omega}\left(\nu^{\prime}, \nu^{\prime \prime}\right)+m_{\Omega}\left(\nu^{\prime}+\nu^{\prime \prime}, \nu^{\prime \prime \prime}\right)=m_{\Omega}\left(\nu^{\prime \prime}, \nu^{\prime \prime \prime}\right)+m_{\Omega}\left(\nu^{\prime}, \nu^{\prime \prime}+\nu^{\prime \prime \prime}\right)$, which is easily verified.) Thus $\left(a^{\prime}, a^{\prime \prime}\right) \rightarrow a^{\prime} \circ a^{\prime \prime}$ defines a structure of associative graded $\mathscr{A}$ algebras on $\mathscr{K}_{\Omega}=\bigoplus_{\nu} \mathscr{K}_{\nu, \Omega}$. (The grading is by elements $\nu \in \mathbf{N}^{I}$.) This algebra has a unit element in $\mathscr{K}_{0, \Omega}$. Note that

(a) $\mathscr{K}_{\Omega}$ has a canonical basis defined by the elements of $\mathscr{P}_{\mathbf{v}, \Omega}$ for various $\mathbf{V} \in \mathscr{V}$.

10.3. Now $D$ (see 3.8) defines an involution of $\mathscr{H}$ as a graded abelian group; from the formulas in 3.8 we see that this is a ring homomorphism that is semilinear with respect to the involution of the ring $\mathscr{A}$ that takes $v$ to $v^{-1}$.

10.4. In the setup of 4.1 , let $\mathscr{K}_{\mathrm{T}, \mathrm{w}, \Omega}$ be the the abelian group with one generator $(L)$ for each isomorphism class of objects of $\mathscr{Q}_{\mathbf{T}, \mathbf{w}, \Omega}$ and with relations $(L)+\left(L^{\prime}\right)=\left(L^{\prime \prime}\right)$ whenever $L^{\prime \prime}$ is isomorphic to $L \oplus L^{\prime}$. We regard $\mathscr{K}_{\mathbf{T}, \mathbf{w}, \Omega}$ as a module over $\mathscr{A}$ by defining $v(L)=(L[1]), v^{-1}(L)=(L[-1])$. It is clearly a free $\mathscr{A}$-module with basis $\left(L_{1} \otimes L_{2}\right)$ where $L_{1}$ (resp. $\left.L_{2}\right)$ runs over $\mathscr{P}_{\mathrm{T}, \Omega}$ (resp. $\mathscr{P}_{\mathrm{W}, \Omega}$ ). Hence the external tensor product defines an isomorphism of $\mathscr{A}$-modules $\mathscr{K}_{\mathrm{T}, \Omega} \otimes_{\mathscr{A}} \mathscr{K}_{\mathrm{W}, \Omega} \cong \mathscr{K}_{\mathrm{T}, \mathrm{W}, \Omega}$.

The functor $\operatorname{res}_{\mathrm{T}, \mathrm{w}}: \mathscr{Q}_{\mathbf{V}, \Omega} \rightarrow \mathscr{Q}_{\mathrm{T}, \mathrm{w}, \Omega}$ is additive; hence it induces a homomorphism res $_{\mathrm{T}, \mathrm{w}}: \mathscr{K}_{\mathrm{V}, \Omega} \rightarrow \mathscr{K}_{\mathrm{T}, \mathrm{w}, \Omega}$. This can be regarded as a homo-

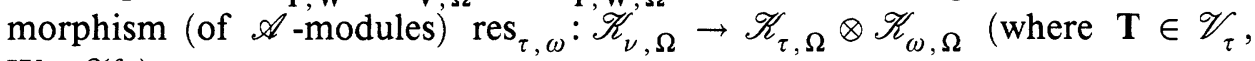
$\left.\mathbf{W} \in \mathscr{V}_{\omega}\right)$.

10.5. We have the following associativity property. Assume that we have $\nu=$ $\sigma+\sigma^{\prime}+\sigma^{\prime \prime}\left(\right.$ in $\left.\mathbf{N}^{I}\right)$. Then the homomorphisms $\left(1 \otimes \operatorname{res}_{\sigma^{\prime}, \sigma^{\prime \prime}}\right) \operatorname{res}_{\sigma, \sigma^{\prime}+\sigma^{\prime \prime}}$ and $\left(\right.$ res $\left._{\sigma, \sigma^{\prime}} \otimes 1\right)$ res $_{\sigma+\sigma^{\prime}, \sigma^{\prime \prime}}$ from $\mathscr{K}_{\nu, \Omega}$ to $\mathscr{K}_{\sigma, \Omega} \otimes \mathscr{K}_{\sigma^{\prime}, \Omega} \otimes \mathscr{K}_{\sigma^{\prime \prime}, \Omega}$ coincide. (See 4.10.) 
10.6. Let $\nu^{\prime}, \nu^{\prime \prime}, \tau, \omega \in \mathbf{N}^{I}$ be such that $\nu^{\prime}+\nu^{\prime \prime}=\tau+\omega$. Let $L^{\prime} \in \mathscr{K}_{\nu^{\prime}, \Omega}$, $L^{\prime \prime} \in \mathscr{K}_{\nu^{\prime \prime}, \Omega}$. The following equality holds in $\mathscr{K}_{\tau, \Omega} \otimes \mathscr{K}_{\omega, \Omega}$ :

$$
\operatorname{res}_{\tau, \omega}\left(L^{\prime} \circ L^{\prime \prime}\right)=\sum_{\kappa} v^{f(\kappa)} \operatorname{res}_{\alpha^{\prime}, \beta^{\prime}}\left(L^{\prime}\right) \circ \operatorname{res}_{\alpha^{\prime \prime}, \beta^{\prime \prime}}\left(L^{\prime \prime}\right)
$$

where the sum is taken over all $\kappa=\left(\alpha^{\prime}, \beta^{\prime}, \alpha^{\prime \prime}, \beta^{\prime \prime}\right) \in \mathscr{N}$, notation of 8.3 (thus, $\nu^{\prime}=\alpha^{\prime}+\beta^{\prime}, \nu^{\prime \prime}=\alpha^{\prime \prime}+\beta^{\prime \prime}, \tau=\alpha^{\prime}+\alpha^{\prime \prime}, \omega=\beta^{\prime}+\beta^{\prime \prime}$ ), and

$$
f(\kappa)=\sum_{h \in \Omega}\left(\beta^{\prime}\left(h^{\prime}\right) \alpha^{\prime \prime}\left(h^{\prime \prime}\right)-\alpha^{\prime}\left(h^{\prime}\right) \beta^{\prime \prime}\left(h^{\prime \prime}\right)\right)+\sum_{i \in I}\left(\alpha^{\prime}(i) \beta^{\prime \prime}(i)-\beta^{\prime}(i) \alpha^{\prime \prime}(i)\right) .
$$

(By definition, $\left(a_{1} \otimes a_{2}\right) \circ\left(a_{3} \otimes a_{4}\right)=\left(a_{1} \circ a_{3}\right) \otimes\left(a_{2} \circ a_{4}\right)$ for $a_{1} \in \mathscr{K}_{\alpha^{\prime}, \Omega}$, $a_{2} \in \mathscr{K}_{\beta^{\prime}, \Omega}, a_{3} \in \mathscr{K}_{\alpha^{\prime \prime}, \Omega}, a_{4} \in \mathscr{K}_{\beta^{\prime \prime}, \Omega}$.) This follows from 8.4 and the identity

$$
f(\kappa)=-2 g(\kappa)+m_{\Omega}\left(\alpha^{\prime}+\beta^{\prime}, \alpha^{\prime \prime}+\beta^{\prime \prime}\right)-m_{\Omega}\left(\alpha^{\prime}, \alpha^{\prime \prime}\right)-m_{\Omega}\left(\beta^{\prime}, \beta^{\prime \prime}\right) .
$$

10.7. We consider the abelian group $\mathbf{Z}^{I}$ of all functions $I \rightarrow \mathbf{Z}$ (with the pointwise sum operation); this contains $\mathbf{N}^{Z}$ as a submonoid. Let $\Gamma$ be the group algebra of this group with coefficients in $\mathscr{A}$; thus, $\Gamma$ has an $\mathscr{A}$-basis $\left\{K_{\alpha} \mid \alpha \in \mathbf{Z}^{I}\right\}$ and $K_{\alpha} K_{\alpha^{\prime}}=K_{\alpha+\alpha^{\prime}}$. Let $\Gamma K_{\Omega}$ be the $\mathscr{A}$-module $\Gamma \otimes_{\mathscr{A}} \mathscr{K}_{\Omega}$.

Proposition 10.8. There is a unique associative $\mathscr{A}$-algebra structure on $\Gamma K_{\Omega}$ such that

$$
\left(K_{\alpha} \otimes L\right)\left(K_{\alpha^{\prime}} \otimes L^{\prime}\right)=v^{r\left(\beta, \alpha^{\prime}\right)} K_{\alpha+\alpha^{\prime}} \otimes\left(L \circ L^{\prime}\right)
$$

for any $L \in \mathscr{K}_{\beta, \Omega}, L^{\prime} \in \mathscr{K}_{\beta^{\prime}, \Omega}$, and any $\alpha, \alpha^{\prime} \in \mathbf{Z}^{I}$, where

$$
r\left(\beta, \alpha^{\prime}\right)=-\sum_{h \in H} \beta\left(h^{\prime}\right) \alpha^{\prime}\left(h^{\prime \prime}\right)+2 \sum_{i \in I} \beta(i) \alpha^{\prime}(i) .
$$

This follows from the associativity of the product $L \circ L^{\prime}$ and the identity

$$
r\left(\beta, \alpha^{\prime}\right)+r\left(\beta+\beta^{\prime}, \alpha^{\prime \prime}\right)=r\left(\beta^{\prime}, \alpha^{\prime \prime}\right)+r\left(\beta, \alpha^{\prime}+\alpha^{\prime \prime}\right) .
$$

10.9. We shall write $K_{\alpha} L$ instead of $K_{\alpha} \otimes L \in \Gamma K_{\Omega}$. The algebra structure 10.8 on $\Gamma K_{\Omega}$ gives rise in the usual way to an algebra structure on $\Gamma K_{\Omega} \otimes_{\mathscr{A}} \Gamma K_{\Omega}$. We have the following result.

Proposition 10.10. There is a unique coassociative $\mathscr{A}$-coalgebra structure on $\Gamma K_{\Omega}$ with comultiplication $\Delta: \Gamma K_{\Omega} \rightarrow \Gamma K_{\Omega} \otimes \Gamma K_{\Omega}$ such that for any $L \in \mathscr{K}_{\nu, \Omega}$ and any $\sigma \in \mathbf{Z}^{I}$ we have

$$
\Delta\left(K_{\sigma} L\right)=\sum_{\tau, \omega} v^{s(\tau, \omega)}\left(K_{\sigma} \otimes K_{\sigma-\tau}\right) \operatorname{res}_{\tau, \omega}(L)
$$

where the sum is taken over all $\tau, \omega$ in $\mathbf{N}^{I}$ such that $\tau+\omega=\nu$; the last product is computed in the algebra $\Gamma K_{\Omega} \otimes_{\mathscr{A}} \Gamma K_{\Omega}$; we have

$$
s(\tau, \omega)=-\sum_{h \in \Omega} \tau\left(h^{\prime}\right) \omega\left(h^{\prime \prime}\right)+\sum_{i \in I} \tau(i) \omega(i) .
$$

This follows from the associativity property 10.5 of res and the identity

$$
s\left(\gamma+\gamma^{\prime}, \gamma^{\prime \prime}\right)+s\left(\gamma, \gamma^{\prime}\right)=s\left(\gamma, \gamma^{\prime}+\gamma^{\prime \prime}\right)+s\left(\gamma^{\prime}, \gamma^{\prime \prime}\right) .
$$


Proposition 10.11. The comultiplication $\Delta: \Gamma K_{\Omega} \rightarrow \Gamma K_{\Omega} \otimes \Gamma K_{\Omega}$ is an algebra homomorphism.

Let $L^{\prime} \in \mathscr{K}_{\nu^{\prime}, \Omega}, L^{\prime \prime} \in \mathscr{K}_{\nu^{\prime \prime}, \Omega}$, and let $\sigma^{\prime}, \sigma^{\prime \prime}$ be in $\mathbf{Z}$. Let $\nu=\nu^{\prime}+\nu^{\prime \prime}$. Recall from 10.2 that $L^{\prime} \circ L^{\prime \prime}=v^{m_{\Omega}\left(\nu^{\prime}, \nu^{\prime \prime}\right)} L^{\prime} * L^{\prime \prime}$. By $10.6,10.8,10.10$, we have

$$
\begin{aligned}
& \Delta\left(K_{\sigma^{\prime}} L^{\prime} K_{\sigma^{\prime \prime}} L^{\prime \prime}\right) \\
& \quad=\Delta\left(v^{r\left(\nu^{\prime}, \sigma^{\prime \prime}\right)} K_{\sigma^{\prime}+\sigma^{\prime \prime}}\left(L^{\prime} \circ L^{\prime \prime}\right)\right) \\
& \quad=\sum_{\tau, \omega} v^{r\left(\nu^{\prime}, \sigma^{\prime \prime}\right)+s(\tau, \omega)}\left(K_{\sigma^{\prime}+\sigma^{\prime \prime}} \otimes K_{\sigma^{\prime}+\sigma^{\prime \prime}-\tau}\right) \operatorname{res}_{\tau, \omega}\left(L^{\prime} \circ L^{\prime \prime}\right) \\
& \quad=\sum_{\kappa} v^{r\left(\nu^{\prime}, \sigma^{\prime \prime}\right)+s(\tau, \omega)+f(\kappa)}\left(K_{\sigma^{\prime}+\sigma^{\prime \prime}} \otimes K_{\sigma^{\prime}+\sigma^{\prime \prime}-\tau}\right) \operatorname{res}_{\alpha^{\prime}, \beta^{\prime}}\left(L^{\prime}\right) \circ \operatorname{res}_{\alpha^{\prime \prime}, \beta^{\prime \prime}}\left(L^{\prime \prime}\right)
\end{aligned}
$$

(in the first sum, $\tau, \omega \in \mathbf{N}^{I}$ are subject to $\tau+\omega=\nu$; in the second $\kappa=$ $\left(\alpha^{\prime}, \beta^{\prime}, \alpha^{\prime \prime}, \beta^{\prime \prime}\right)$ is subject to $\left.\nu^{\prime}=\alpha^{\prime}+\beta^{\prime}, \nu^{\prime \prime}=\alpha^{\prime \prime}+\beta^{\prime \prime}\right)$.

On the other hand, we have

$$
\begin{aligned}
& \Delta\left(K_{\sigma^{\prime}} L^{\prime}\right) \Delta\left(K_{\sigma^{\prime \prime}} L^{\prime \prime}\right) \\
& =\sum_{\kappa} v^{s\left(\alpha^{\prime}, \beta^{\prime}\right)+s\left(\alpha^{\prime \prime}, \beta^{\prime \prime}\right)}\left(K_{\sigma^{\prime}} \otimes K_{\sigma^{\prime}-\alpha^{\prime}}\right) \\
& \quad \times \operatorname{res}_{\alpha^{\prime}, \beta^{\prime}}\left(L^{\prime}\right)\left(K_{\sigma^{\prime \prime}} \otimes K_{\sigma^{\prime \prime}-\alpha^{\prime \prime}}\right) \operatorname{res}_{\alpha^{\prime \prime}, \beta^{\prime \prime}}\left(L^{\prime \prime}\right) \\
& =\sum_{\kappa} v^{s^{\prime}(\kappa)}\left(K_{\sigma^{\prime}+\sigma^{\prime \prime}} \otimes K_{\sigma^{\prime}+\sigma^{\prime \prime}-\alpha^{\prime}-\alpha^{\prime \prime}}\right) \operatorname{res}_{\alpha^{\prime}, \beta^{\prime}}\left(L^{\prime}\right) \circ \operatorname{res}_{\alpha^{\prime \prime}, \beta^{\prime \prime}}\left(L^{\prime \prime}\right)
\end{aligned}
$$

where $\kappa$ is as in the previous formula and $s^{\prime}(\kappa)=s\left(\alpha^{\prime}, \beta^{\prime}\right)+s\left(\alpha^{\prime \prime}, \beta^{\prime \prime}\right)+$ $r\left(\alpha^{\prime}, \sigma^{\prime \prime}\right)+r\left(\beta^{\prime}, \sigma^{\prime \prime}-\alpha^{\prime \prime}\right)$.

It remains to verify the following identity:

$r\left(\nu^{\prime}, \sigma^{\prime \prime}\right)+s(\tau, \omega)+f(\kappa)=s\left(\alpha^{\prime}, \beta^{\prime}\right)+s\left(\alpha^{\prime \prime}, \beta^{\prime \prime}\right)+r\left(\alpha^{\prime}, \sigma^{\prime \prime}\right)+r\left(\beta^{\prime}, \sigma^{\prime \prime}-\alpha^{\prime \prime}\right)$ where $\tau=\alpha^{\prime}+\alpha^{\prime \prime}, \omega=\beta^{\prime}+\beta^{\prime \prime}$ and $\nu^{\prime}=\alpha^{\prime}+\alpha^{\prime \prime}$. Since $r(),, s($,$) are$ bilinear, this is equivalent to the identity

$$
s\left(\alpha^{\prime}, \beta^{\prime \prime}\right)+s\left(\alpha^{\prime \prime}, \beta^{\prime}\right)=r\left(\beta^{\prime}, \alpha^{\prime \prime}\right)+f(\kappa) .
$$

which is easily verified.

10.12. For each $i \in I$ and $a \in \mathbf{N}$ we denote by $F_{i}^{(a)}$ the canonical basis element of $\mathscr{K}_{\mu, \Omega}$ where $\mu$ is such that $\mu(i)=a$ and $\mu(j)=0$ for all $j \neq i$. (This corresponds to the complex 1 on the zero vector space $\mathbf{E}_{\mathbf{v}, \Omega}$, where $\mathbf{W} \in \mathscr{V}_{\mu}$.) Note that $F_{i}^{(0)}$ is the unit element of the algebra $\mathscr{K}_{\Omega}$. It is clear that

$$
D\left(F_{i}^{(a)}\right)=F_{i}^{(a)}
$$

where $D$ is as in 10.3 . 
To state the following result we introduce some notation on Gaussian binomial coefficients:

$$
[a]_{!}=\prod_{k=1}^{a} \frac{v^{k}-v^{-k}}{v-v^{-1}}, \quad\left[a, a^{\prime}\right]=\frac{\left[a+a^{\prime}\right]_{!}}{[a]_{!}\left[a^{\prime}\right]_{!}} .
$$

Proposition 10.13. (a) For any (i, a) $\in S_{\nu}$ (with $m$ terms each) we have the following equalities in $\mathscr{K}_{\Omega}$ :

$$
D\left(L_{\mathbf{i}, \mathbf{a}, \Omega}\right)=v^{2 d(\mathbf{i}, \mathbf{a})} L_{\mathbf{i}, \mathbf{a}, \Omega}
$$

where $d(\mathbf{i}, \mathbf{a})$ is as in $3.8(\mathbf{b})$ and

$$
L_{\mathbf{i}, \mathbf{a}, \Omega}=v^{d(\mathbf{i}, \mathbf{a})} F_{i_{1}}^{\left(a_{1}\right)} \circ F_{i_{2}}^{\left(a_{2}\right)} \circ \cdots \circ F_{i_{m}}^{\left(a_{m}\right)} .
$$

(D is as in 10.3.)

(b) The elements $F_{i}^{(a)} \quad(i \in I, a \in \mathbf{N})$ generate the $\mathscr{A}$-algebra $\mathscr{K}_{\Omega}$.

(c) If $i \in I$ and $a, a^{\prime} \in \mathbf{N}$, we have

$$
F_{i}^{(a)} \circ F_{i}^{\left(a^{\prime}\right)}=\left[a, a^{\prime}\right] F_{i}^{\left(a+a^{\prime}\right)}
$$

in $\mathscr{K}_{\Omega}$.

(d) Let $i, j$ be distinct elements of $I$ and let $N$ be the number of edges joining them in our graph. We have the following equality in $\mathscr{K}_{\Omega}$ :

$$
\sum_{p=0}^{N+1}(-1)^{p} F_{i}^{(p)} \circ F_{j}^{(1)} \circ F_{i}^{(N+1-p)}=0 .
$$

The first formula in (a) follows from 3.8(a).

From 3.7(a) we see that the two sides of the second formula in (a) are equal for a certain unknown value for the exponent of $v$. The value of that exponent can be determined by applying the ring involution $D$ to the two sides of that equality, using the first formula in (a) and 10.12(a).

Now (b) follows from (a) and from 7.3. The equality (c) follows from (a) and 2.4(a); (d) follows from (a) and 9.5.

Proposition 10.14. Let $\Omega^{\prime}$ be a second orientation of our graph. Assume that $\mathbf{k}$ is as in 5.1. Then $\mathscr{F}\left(\right.$ see 5.2, 5.6) defines an $\mathscr{A}$-linear isomorphism $\mathscr{K}_{\Omega} \cong \mathscr{K}_{\Omega^{\prime}}$ (preserving the grading) which will be denoted again by $\mathscr{F}$.

(a) $\mathscr{F}: \mathscr{K}_{\Omega} \cong \mathscr{K}_{\Omega^{\prime}}$ is an algebra isomorphism. It takes the canonical basis (10.2(a)) of $\mathscr{K}_{\Omega}$ onto the canonical basis of $\mathscr{K}_{\Omega^{\prime}}$.

(b) There is a unique algebra homomorphism $\Gamma K_{\Omega} \cong \Gamma K_{\Omega^{\prime}}$ that takes $K_{\alpha} L$ to $K_{\alpha} \mathscr{F}(L)$ for any $\alpha \in \mathbf{Z}^{I}$ and any $L \in \mathscr{K}_{\Omega}$. This is compatible with the comultiplication.

(a) follows from 5.4, 5.5, 5.6. The first statement of (b) follows from (a). To verify the compatibility in the second statement, it is enough to verify it on the algebra generators of $\mathscr{K}_{\Omega}$ (see $\left.10.13(b)\right)$ and on the generators of $\Gamma$, where it is obvious. 
10.15. Let $u^{-}$be the - part of the enveloping algebra $u$ of the Lie algebra (over $\mathbf{Q}$ ) attached by Kac and Moody to the generalized symmetric Cartan matrix $\left(a_{i j}\right)$ indexed by $I \times I$ where $a_{i i}=2$ and $-a_{i j}$ is the number of edges joining $i, j$ in the graph, for $i \neq j$. This is the $\mathbf{Q}$-algebra defined by generators $F_{i} \quad(i \in I)$ and relations

$$
\sum_{p=0}^{N+1}(-1)^{p}\left(\begin{array}{c}
N+1 \\
p
\end{array}\right) F_{j}^{p} F_{i} F_{j}^{N+1-p}=0
$$

for any $i \neq j$ (with $N=-a_{i j}$ ).

Let $\mathbf{U}^{-}$be the - part of the quantized enveloping algebra $\mathbf{U}$ (over $A^{\prime}$, the quotient field of $\mathscr{A}$ ) attached by Drinfeld and Jimbo to the same Cartan matrix. This is the $A^{\prime}$-algebra defined by generators $F_{i} \quad(i \in I)$ and relations

$$
\sum_{p=0}^{N+1}[p, N+1-p] F_{j}^{p} F_{i} F_{j}^{N+1-p}=0
$$

for any $i \neq j$ (with $N=-a_{i j}$ ). ( $\mathbf{U}$ itself has additional generators $K_{i}, K_{i}^{-1}$, $E_{i}(i \in I)$.)

We shall regard $\mathbf{U}^{-}$as a $\mathbf{N}^{I}$-graded algebra; the grading $\mathbf{U}^{-}=\bigoplus_{\nu} \mathbf{U}_{\nu}^{-}$is uniquely defined by the condition that for all $i \in I$ we have $F_{i} \in \mathbf{U}_{\mu}^{-}$where $\mu(i)=1$ and $\mu(j)=0$ for $j \neq i$.

Note that $\mathbf{U}_{\nu}^{-}$is a finite-dimensional $A^{\prime}$-vector space for any $\nu \in \mathbf{N}^{I}$.

Similarly, $u^{-}$is a graded $\mathbf{N}^{I}$-algebra.

10.16. From 10.13 (c),(d) we see that there exists a unique $A^{\prime}$-algebra homomorphism $\lambda_{\Omega}: \mathbf{U}^{-} \rightarrow \mathscr{K}_{\Omega} \otimes A^{\prime}$ such that $\lambda_{\Omega}\left(F_{i}\right)=F_{i}^{(1)}$ for all $i \in I$.

Theorem 10.17. (a) $\lambda_{\Omega}$ is an isomorphism of $A^{\prime}$-algebras.

(b) Let $\Omega^{\prime}$ be a second orientation of our graph. Then $\lambda_{\Omega^{\prime}}=\mathscr{F} \lambda_{\Omega}$ where $\mathscr{F}$ is as in 10.14(a).

(c) Let $\mathbf{B}$ be the inverse image under $\lambda_{\Omega}$ of the canonical basis of $\mathscr{K}_{\Omega}$. Then $\mathbf{B}$ is an $A^{\prime}$-basis of $\mathbf{U}^{-}$that is independent of $\Omega$. (We call it the canonical basis of $\mathbf{U}^{-}$.)

First note that (b) holds: it is enough to verify the equality in (b) on the algebra generators $F_{i}$, where it is obvious.

In the rest of the proof we assume that, for a particular orientation $\Omega$, we have

$$
\operatorname{dim}_{\mathbf{Q}} u_{\nu}^{-} \leq \operatorname{dim}_{\mathbf{Q}} \mathscr{K}_{\nu, \Omega} \otimes \mathbf{Q}
$$

for all $\nu$. (Here, $\mathbf{Q}$ is regarded as a $\mathscr{A}$-algebra with $v \rightarrow-1$.)

We now prove (a). Note that $\lambda_{\Omega}$ is compatible with the $\mathbf{N}^{I}$-gradings, that the homogeneous components of these gradings are finite-dimensional, and that $\lambda_{\Omega}$ is surjective (see 10.13(b),(c)). 
Hence to prove (a) it is enough to prove that

$$
\operatorname{dim}_{A^{\prime}} \mathbf{U}_{\nu}^{-} \leq \operatorname{dim}_{A^{\prime}} \mathscr{K}_{\nu, \Omega} \otimes A^{\prime}
$$

for any $\nu$. By general principles, it is enough to verify this in the case where $\mathbf{k}$ is as in 5.1. In that case, $\operatorname{dim}_{A^{\prime}} \mathscr{K}_{\nu, \Omega} \otimes A^{\prime}$ is independent of the choice of orientation, by $10.14(\mathrm{a})$. Hence it is enough to verify (e) for a particular orientation $\Omega$, for example for one such that (d) holds.

Since $u^{-}$is a specialization of $\mathbf{U}^{-}$for $v=1$, we have $\operatorname{dim}_{A^{\prime}} \mathbf{U}_{\nu}^{-} \leq$ $\operatorname{dim}_{\mathbf{Q}} u_{\nu}^{-} ;$hence $(\mathrm{d})$ implies

$$
\operatorname{dim}_{A^{\prime}} \mathbf{U}_{\nu}^{-} \leq \operatorname{dim}_{\mathbf{Q}} \mathscr{K}_{\nu, \Omega} \otimes \mathbf{Q}=\operatorname{dim}_{A^{\prime}} \mathscr{K}_{\nu, \Omega} \otimes A^{\prime}
$$

for any $\nu$. This proves (a).

To prove (c), we may again assume by general principles that $\mathbf{k}$ is as in 5.1 . In that case, (c) follows immediately from (a),(b) and 10.14(a). This completes the proof, except for the verification of $(d)$; that verification will be done in 10.21 .

10.18. Let $X$ be a variety over $\mathbf{k}$. Let $M(X)$ be the $\mathbf{Q}$-vector space of all constructible functions $f: X \rightarrow \mathbf{Q}$, that is, of all functions such that $f^{-1}(a)$ is constructible for any $a \in \mathbf{Q}$ and is empty for all but finitely many $a$.

Following MacPherson [M], for any morphism $m: X \rightarrow X^{\prime}$ of varieties we define linear maps $m^{*}: M\left(X^{\prime}\right) \rightarrow M(X)$ and $m_{1}: M(X) \rightarrow M\left(X^{\prime}\right)$ by $\left(m^{*} f^{\prime}\right)(x)=f^{\prime}(m(x)),\left(m_{!} f\right)\left(x^{\prime}\right)=\sum_{a \in \mathbf{Q}} a \chi\left(m^{-1}\left(x^{\prime}\right) \cap f^{-1}(a)\right)$, where $\chi$ denotes Euler characteristic in $l$-adic cohomology with compact support.

These operations are related to the analogous operations in derived category as follows.

If $L \in \mathscr{D}(X)$, we can attach to $L$ the function $f_{L} \in M(X)$ defined by $f_{L}(x)=\sum_{j}(-1)^{j} \operatorname{dim} \mathscr{H}_{x}^{i} L$ where $\mathscr{H}_{x}^{i} L$ are the stalks of the cohomology sheaves of $L$ at $x \in X$. Now let $L^{\prime} \in \mathscr{D}\left(X^{\prime}\right)$. We then have $m_{!} f_{L}=f_{m_{1} L}$ and $m^{*} f_{L^{\prime}}=f_{m^{*} L^{\prime}}$. We also have $f_{L[d]}=(-1)^{d} f_{L}$ for any integer $d$.

10.19. F or any $\mathbf{V} \in \mathscr{V}$, we define $\mathscr{M}(\mathbf{V}, \Omega)$ to be the vector space of all functions in $M\left(\mathbf{E}_{\mathbf{V}, \Omega}\right)$ that are constant on the orbits of $G_{\mathbf{V}}$. Now let $\mathbf{V}, \mathbf{V}^{\prime}, \mathbf{V}^{\prime \prime}$ be as in 3.1, and let $f^{\prime} \in M\left(\mathbf{E}_{\mathbf{V}^{\prime}, \Omega}\right), f^{\prime \prime} \in M\left(\mathbf{E}_{\mathbf{V}^{\prime \prime}, \Omega}\right)$. We define a function $f^{\prime} * f^{\prime \prime} \in M\left(\mathbf{E}_{\mathbf{V}, \Omega}\right)$ formally as in 3.1. We shall use the notations of 3.1 . Let $f_{1} \in M\left(\mathbf{E}_{\mathbf{v}^{\prime}, \Omega} \times \mathbf{E}_{\mathbf{v}^{\prime \prime}, \Omega}\right)$ be given by $f_{1}\left(x^{\prime}, x^{\prime \prime}\right)=f^{\prime}\left(x^{\prime}\right) f^{\prime \prime}\left(x^{\prime \prime}\right)$. Then there is a unique function $f_{3} \in M\left(\mathbf{E}^{\prime \prime}\right)$ such that $p_{1}^{*} f_{1}=p_{3}^{*} f_{3}$; by definition, $f^{\prime} * f^{\prime \prime}=\left(p_{3}\right)_{!}\left(f_{3}\right)$.

Next we note that given $\nu \in \mathbf{N}^{I}$, the vector spaces $\mathscr{M}(\mathbf{V}, \Omega)$ for various $\mathbf{V} \in$ $\mathscr{V}_{\nu}$ can all be identified in a coherent way with a single vector space $\mathscr{M}(\nu, \Omega)$ (by the invariance condition on the functions considered). The operation above 
becomes a pairing $\mathscr{M}\left(\nu^{\prime}, \Omega\right) \times \mathscr{M}\left(\nu^{\prime \prime}, \Omega\right) \rightarrow \mathscr{M}(\nu, \Omega)$ denoted $\left(f^{\prime}, f^{\prime \prime}\right) \rightarrow$ $f^{\prime} * f^{\prime \prime}$. Let $\mathscr{M}(\Omega)=\bigoplus_{\nu} \mathscr{M}(\nu, \Omega)$. The operation $*$ makes $\mathscr{M}(\Omega)$ into an associative Q-algebra.

Let $i \in I$; let $\mu$ be such that $\mu(i)=1$ and $\mu(j)=0$ for $j \neq i$. Then $\mathscr{M}(\mu, \Omega)$ is one dimensional, with a canonical basis element $F_{i}$ corresponding to the function on $\{0\}$ with value 1 . Let $\mathscr{M}_{0}(\Omega)$ be the subalgebra of $\mathscr{M}(\Omega)$ generated by the elements $F_{i} \quad(i \in I)$, and let $\left.\mathscr{M}_{0}(\nu, \Omega)=\mathscr{M}_{(} \nu, \Omega\right) \cap \mathscr{M}_{0}(\Omega)$. Then $\mathscr{M}_{0}(\Omega)=\bigoplus_{\nu} \mathscr{M}_{0}(\nu, \Omega)$.

The definition of $\mathscr{M}_{0}(\Omega)$ given above is a reformulation of a definition given by Schofield in [S] (which is itself a variant of a construction of Ringel [R]). We shall recall the definition of $[\mathrm{S}]$ in a slightly different form, more convenient for our purposes.

Let $S_{\nu}^{0}$ be the set of all sequences $\mathbf{i}$ such that $(\mathbf{i}, \mathbf{a}) \in S_{\nu}$ where a is a sequence of form $(1,1, \ldots, 1)$.

Let $R$ be the Q-vector space with basis indexed by the elements $\mathbf{i}$ in $\bigsqcup_{\nu} S_{\nu}^{0}$. We regard $R$ as an associative algebra with product $\mathbf{i}^{\prime} \mathbf{i}^{\prime \prime}$ as in $3.2(\mathrm{~b})$.

For any $\mathbf{V} \in \mathscr{V}_{\nu}$ and any $x \in \mathbf{E}_{\mathbf{V}, \Omega}$ we define a linear form $t_{x}: R \rightarrow \mathbf{Q}$ by $t_{x}(\mathbf{i})=0$ if $\mathbf{i} \in S_{\nu^{\prime}}^{0}$ with $\nu^{\prime} \neq \nu$ and $\left.t_{x}(\mathbf{i})=\chi\left(\pi_{\mathbf{i}, \mathbf{a}}\right)^{-1}(x)\right)$ if $\mathbf{i} \in S_{\nu}^{0}$; here $(\mathbf{a})=(1,1, \ldots 1)$ and $\pi_{\mathbf{i}, \mathbf{a}}$ is as in 1.5. Let $\mathscr{I}$ be the intersection of the kernels of $t_{x}$ for various $\mathbf{V}, x$ as above. One shows that $\mathscr{I}$ is a two-sided ideal in $R$, and one defines $\mathscr{M}_{0}^{\prime}(\Omega)$ as the Q-algebra $R / \mathscr{I}$. We define a Q-linear map $R \rightarrow \mathscr{M}(\Omega)$ by associating to a basis element $\mathbf{i}$ of $R$ the constructible function $x \rightarrow t_{x}(\mathbf{i})$ on $\mathbf{E}_{\mathbf{V}, \Omega}$ (where $\mathbf{i} \in S_{\nu}^{0}$ and $\mathbf{V} \in \mathscr{V}_{\nu}$ ). It is clear that the kernel of this map is exactly $\mathscr{I}$ and its image is exactly $\mathscr{M}_{0}(\Omega)$. Hence it defines an isomorphism

$$
\mathscr{M}_{0}^{\prime}(\Omega) \cong \mathscr{M}_{0}(\Omega)
$$

compatible with multiplication. We shall denote the element of $\mathscr{M}_{0}^{\prime}(\Omega)$ corresponding to $F_{i} \in \mathscr{M}_{0}(\Omega)$, again by $F_{i}$.

The following proposition is proved in [S].

Proposition 10.20. Assume that $\Omega$ has no cycles or, in other words, that we cannot find $m \geq 2$ and $h_{1}, h_{2}, \ldots, h_{m}$ in $\Omega$ such that $h_{i}^{\prime \prime}=h_{i+1}^{\prime}$ for $1 \leq i<m$ and $h_{m}^{\prime \prime}=h_{1}^{\prime}$. Then there is a unique Q-algebra isomorphism $u^{-} \cong \mathscr{M}_{0}^{\prime}(\Omega)$ under which $F_{i}$ corresponds to $F_{i}$ for all $i \in I$.

10.21. We define a new product $\mathscr{M}\left(\nu^{\prime}, \Omega\right) \times \mathscr{M}\left(\nu^{\prime \prime}, \Omega\right) \rightarrow \mathscr{M}(\nu, \Omega)$ by $\left(f^{\prime}, f^{\prime \prime}\right)$ $\rightarrow f^{\prime} \circ f^{\prime \prime}=(-1)^{m_{\Omega}\left(\nu^{\prime}, \nu^{\prime \prime}\right)} f^{\prime} * f^{\prime \prime}$. This defines a new associative algebra structure on $\mathscr{M}(\Omega)$ for which the subalgebra generated by the $F_{i}$ is the same subspace $\mathscr{M}_{0}(\Omega)$, but with a new multiplication.

Let $\mathbf{V} \in \mathscr{V}_{\nu}$. The correspondence $L \rightarrow f_{L}$ (see 10.18) defines a homomorphism of abelian groups $\mathscr{K}_{\mathbf{V}, \Omega} \rightarrow \mathscr{M}(\mathbf{V}, \Omega)$. This extends uniquely to a $\mathbf{Q}-$ linear map $\mathscr{K}_{\mathbf{V}, \Omega} \otimes \mathbf{Q} \rightarrow \mathscr{M}(\mathbf{V}, \Omega)$, where $\mathbf{Q}$ is regarded as a $\mathscr{A}$-module with $v$ acting as -1 . This may be regarded as a $\mathbf{Q}$-linear map $\mathscr{K}_{\nu, \Omega} \otimes \mathbf{Q} \rightarrow \mathscr{M}(\nu, \Omega)$; hence it gives rise to a $\mathbf{Q}$-linear map $\mathscr{K}_{\Omega} \otimes \mathbf{Q} \rightarrow \mathscr{M}(\Omega)$. From the definitions 
and from the results in 10.18 , we see that this is a $\mathbf{Q}$-algebra homomorphism (for the new algebra structure on $\mathscr{M}(\Omega)$ ). It clearly takes $F_{i}^{(1)}$ to $F_{i}$, and hence, by $10.13(b),(c)$ its image is exactly $\mathscr{M}_{0}(\Omega)$.

It follows that

$$
\operatorname{dim}_{\mathbf{Q}} \mathscr{K}_{\nu, \Omega} \otimes \mathbf{Q} \geq \operatorname{dim}_{\mathbf{Q}} \mathscr{M}_{0}(\nu, \Omega)
$$

for all $\nu$. It is easy to see that our graph has at least one orientation $\Omega$ for which there are no cycles. For such $\Omega$ we can combine (a) with 10.19(a) and with the conclusion of 10.20 and we see that 10.17 (d) holds. (Note that the isomorphisms in 10.19(a) and 10.20 are compatible with natural gradings.) Thus, Theorem 10.17 is proved.

10.22. Let $\mathbf{U}^{\leq 0}$ be the $A^{\prime}$-vector space $\Gamma \otimes_{A^{\prime}} \mathbf{U}^{-}$. According to Drinfeld and Jimbo, this is a Hopf algebra with multiplication such that $\mathbf{U}^{-}, \Gamma \otimes A^{\prime}$ are subalgebras, and $K_{\alpha} F_{i}=v^{s} F_{i} K_{\alpha}$, where $i \in I, \alpha \in \mathbf{Z}^{I}, s=\sum_{h \in H ; h^{\prime}=i} \alpha\left(h^{\prime \prime}\right)-$ $2 \alpha(i)$. The comultiplication is given by

$$
\Delta\left(F_{i}\right)=1 \otimes F_{i}+F_{i} \otimes K_{\alpha_{i}}^{-1}, \quad \Delta\left(K_{\alpha}\right)=K_{\alpha} \otimes K_{\alpha}
$$

where $\alpha_{i}$ has value 1 at $i$ and value zero at any $j \neq i$.

The isomorphism in 10.16 can be extended to an isomorphism of $A^{\prime}$-vector spaces

by $K_{\alpha} \otimes z \rightarrow K_{\alpha} \lambda_{\Omega}(z)$.

$$
\tilde{\lambda}_{\Omega}: \mathbf{U}^{\leq 0} \cong \Gamma K_{\Omega} \otimes A^{\prime}
$$

Proposition 10.23. (a) $\tilde{\lambda}_{\Omega}$ is an algebra isomorphism.

(b) $\tilde{\lambda}_{\Omega}$ is compatible with the comultiplication.

(a) follows from 10.8 and the definition of $\lambda_{\Omega}$. By 10.11, it is enough to check (b) on a set of algebra generators where it is obvious.

10.24. If $\mathbf{V} \in \mathscr{V}_{\nu}$ and $\mathbf{V}^{*}$ is the dual space, we have an isomorphism $\rho: \mathbf{E}_{\mathbf{V}, \Omega} \cong$ $\mathbf{E}_{\mathbf{V}^{*}, \bar{\Omega}}$ given by $\rho(x)=x^{\prime}$ where $x_{h}^{\prime}: \mathbf{V}_{h^{\prime}}^{*} \rightarrow \mathbf{V}_{h^{\prime \prime}}^{*}$ is the transpose of $x_{\bar{h}}: \mathbf{V}_{h^{\prime \prime}} \rightarrow$ $\mathbf{V}_{h^{\prime}}$.

This induces an equivalence of categories $\mu_{1}: \mathscr{D}\left(\mathbf{E}_{\mathbf{v}, \Omega}\right) \cong \mathscr{D}\left(\mathbf{E}_{\mathbf{v}^{*}, \bar{\Omega}}\right)$ with inverse $\rho^{*}$.

Lemma 10.25. (a) If (i, $\mathbf{a}) \in S_{\nu}$ we have $\rho_{!} L_{\mathbf{i}, \mathbf{a} ; \Omega} \cong L_{\mathbf{i}^{\prime}, \mathbf{a}^{\prime} ; \bar{\Omega}} \in \mathscr{D}\left(\mathbf{E}_{\mathbf{V}^{*}, \bar{\Omega}}\right)$ where $\left(\mathbf{i}^{\prime}, \mathbf{a}^{\prime}\right)$ are the sequences obtained by reading $(\mathbf{i}, \mathbf{a})$ from right to left.

(b) $\rho_{\text {! }}$ defines a bijection $\mathscr{P}_{\mathbf{v}, \Omega} \cong \mathscr{P}_{\mathbf{v}^{*}, \bar{\Omega}}$ with inverse $\rho^{*}$.

(c) $\rho_{\text {! }}$ defines an equivalence of categories $\mathscr{Q}_{\mathbf{V}, \Omega} \cong \mathscr{Q}_{\mathbf{V}^{*}, \bar{\Omega}}$ with inverse $\rho^{*}$.

The (standard) verification of (a) is left to the reader; (b) follows from (a) and (c) follows from (b).

Lemma 10.26. If $\mathbf{V}, \mathbf{V}^{\prime}, \mathbf{V}^{\prime \prime}$ are as in 3.1 and $L^{\prime}, L^{\prime \prime}$ are as in 3.2, then $\rho_{!}\left(L^{\prime} * L^{\prime \prime}\right) \cong \rho_{!}\left(L^{\prime \prime}\right) * \rho_{!}\left(L^{\prime}\right) \in \mathscr{Q}_{\mathbf{V}^{*}, \bar{\Omega}}$.

The proof is standard; it will be omitted. 
10.27. The operation $\rho_{\text {! }}$ in 10.25 induces a $\mathscr{A}$-linear isomorphism $\mathscr{K}_{\nu, \Omega} \cong$ $\mathscr{K}_{\nu, \bar{\Omega}}$ for any $\nu$; hence it induces a $\mathscr{A}$-linear isomorphism $\mathscr{K}_{\Omega} \cong \mathscr{K}_{\bar{\Omega}}$ (denoted again $\rho_{!}$) that carries the canonical basis of the first space onto the canonical basis of the second space (see 10.25(b)). It transforms the multiplication of the first space to the opposite of the multiplication on the second space. This follows from 10.26 and from the identity

$$
m_{\Omega}\left(\nu^{\prime}, \nu^{\prime \prime}\right)=m_{\bar{\Omega}}\left(\nu^{\prime \prime}, \nu^{\prime}\right) \text {. }
$$

10.28. Let $f: \mathbf{U}^{-} \rightarrow \mathbf{U}^{-}$be the unique $A^{\prime}$-linear isomorphism that takes each $F_{i}$ to itself and transforms the multiplication into the opposite one. We have the equality

$$
\left(\rho_{!} \otimes 1\right) \lambda_{\Omega}=\lambda_{\bar{\Omega}} f: \mathbf{U}^{-} \rightarrow \mathscr{K}_{\bar{\Omega}} \otimes A^{\prime} .
$$

(It is enough to check this equality on the generators $F_{i}$, where it is obvious.)

\section{Properties of the canonical basis $\mathbf{B}$ of $\mathbf{U}^{-}$.}

11.1. In this section, $\Omega$ is fixed, but it is used only in proofs; the results do not refer to it.

Proposition 11.2. For any $\nu \in \mathbf{N}^{I}$, let $\mathbf{B}_{\nu}=\mathbf{B} \cap \mathbf{U}_{\nu}^{-}$. Then $\mathbf{B}=\bigcup_{\nu} \mathbf{B}_{\nu}$ (disjoint union) and each $\mathbf{B}_{\nu}$ is a finite set.

This is obvious.

Theorem 11.3. Let $U^{-}$be the $\mathscr{A}$-subalgebra of $\mathbf{U}^{-}$generated by the elements $\left([r]_{!}\right)^{-1} F_{i}^{r}$ for various $i \in I$ and $r \in \mathbf{N}$. Then $\mathbf{B}$ is an $\mathscr{A}$-basis of $U^{-}$and $\mathbf{B}_{\nu}$ is an $\mathscr{A}$-basis of $U^{-} \cap \mathbf{U}_{\nu}^{-}$for any $\nu$.

Indeed, under $\lambda_{\Omega}, U^{-}$corresponds to $\mathscr{K}_{\Omega}$, regarded as an $\mathscr{A}$-subalgebra of $\mathscr{K}_{\Omega} \otimes A^{\prime}$. (See 10.13(b).)

Proposition 11.4. The $A^{\prime}$-linear isomorphism $f: \mathbf{U}^{-} \cong \mathbf{U}^{-}$(see 10.28) takes $\mathbf{B}$ onto itself.

This is clear from $10.27,10.28$.

Theorem 11.5. (a) Let $b^{\prime}, b^{\prime \prime} \in \mathbf{B}$; let us write their product in $\mathbf{U}^{-}$as $b^{\prime} b^{\prime \prime}=$ $\sum_{b \in \mathbf{B}} f_{b^{\prime}, b^{\prime \prime}, b} b$ with $f_{b^{\prime}, b^{\prime \prime}, b} \in A^{\prime}$. Then $f_{b^{\prime}, b^{\prime \prime}, b} \in \mathbf{N}\left[v, v^{-1}\right]$.

(b) Let $b \in \mathbf{B}_{\nu}$; we have

$$
\Delta(b)=\sum_{\tau, \omega} \sum_{b^{\prime} \in \mathbf{B}_{\tau}, b^{\prime \prime} \in \mathbf{B}_{\omega}} g_{b^{\prime}, b^{\prime \prime}, b} b^{\prime} \otimes K_{-\tau} b^{\prime \prime}
$$

in $\mathbf{U}^{\leq 0} \otimes \mathbf{U}^{\leq 0}$ where $g_{b^{\prime}, b^{\prime \prime}, b} \in \mathbf{N}\left[v, v^{-1}\right]$.

Let $b, b^{\prime}, b^{\prime \prime}$ be as in (a). Let $\mathbf{V}, \mathbf{V}^{\prime}, \mathbf{V}^{\prime \prime}$ be as in 3.1 so that $b, b^{\prime}, b^{\prime \prime}$ correspond respectively to $L, L^{\prime}, L^{\prime \prime}$ in $\mathscr{P}_{\mathbf{v}, \Omega}, \mathscr{P}_{\mathbf{v}^{\prime}, \Omega}, \mathscr{P}_{\mathbf{v}^{\prime \prime}, \Omega}$. Let us write

$$
L^{\prime} * L^{\prime \prime}=\bigoplus_{\widetilde{L} \in \mathscr{P}_{\mathbf{v}, \Omega}, d \in \mathbf{Z}} \bar{Q}_{l}^{N(\widetilde{L}, d)} \otimes \widetilde{L}[d] .
$$


Then from the definitions, we have $f_{b^{\prime}, b^{\prime \prime}, b}=v^{s} \sum_{d} N(L, d) v^{d}$ for some integer $s$ and (a) follows.

Now let $b, b^{\prime}, b^{\prime \prime}$ be as in (b). Let $\mathbf{V}, \mathbf{T}, \mathbf{W}$ be as in 4.1 so that $b, b^{\prime}, b^{\prime \prime}$ correspond respectively to $L, L^{\prime}, L^{\prime \prime}$ in $\mathscr{P}_{\mathbf{V}, \Omega}, \mathscr{P}_{\mathbf{T}, \Omega}, \mathscr{P}_{\mathbf{W}, \Omega}$. Let us write

$$
\operatorname{res}_{\mathbf{T}, \mathbf{W}} L=\bigoplus_{\tilde{L^{\prime} \in \mathscr{P}_{\mathrm{T}, \Omega}}} \bigoplus_{L^{\prime \prime} \in \mathscr{P}_{\mathbf{W}, \Omega}, d \in \mathbf{Z}} \bar{Q}_{l}^{N\left(\widetilde{L^{\prime}}, \widetilde{L^{\prime \prime}}, d\right)} \otimes \widetilde{L^{\prime}} \otimes \widetilde{L^{\prime \prime}}[d] .
$$

Then from the definitions, we have $g_{b^{\prime}, b^{\prime \prime}, b}=v^{s} \sum_{d} N\left(L^{\prime}, L^{\prime \prime}, d\right) v^{d}$ for some integer $s$ and (b) follows.

11.6. Let $b \in \mathbf{B}_{\nu}$ and let $i \in I$. We associate to $b, i$ an integer $s_{i}(b)$ as follows. By definition, $s_{i}(b)$ is the largest integer $r$ such that $0 \leq r \leq \nu(i)$ and such that

(a) there exists $z^{\prime} \in \mathbf{U}^{-}$such that $b$ appears with nonzero coefficient in $F_{i}^{r} z^{\prime}$, expressed as an $A^{\prime}$-linear combination of elements of $\mathbf{B}$ or, equivalently,

(a1) there exists $b^{\prime} \in \mathbf{B}$ such that $b$ appears with non-zero coefficient in $F_{i}^{r} b^{\prime}$, expressed as an $\mathscr{A}$-linear combination of elements of $\mathbf{B}$.

(This is well defined since (a) is satisfied with $r=0$.)

Using 6.6 for our $\Omega$, we see that

(b) there exist elements $z_{r^{\prime}}^{\prime} \in \mathbf{U}^{-} \quad\left(s_{i}(b)<r^{\prime} \leq \nu(i)\right)$ and $z_{r^{\prime}}^{\prime \prime} \in \mathbf{U}^{-} \quad\left(s_{i}(b) \leq\right.$ $\left.r^{\prime} \leq \nu(i)\right)$ such that

$$
b+\sum_{r^{\prime}>s_{i}(b)} F_{i}^{r^{\prime}} z_{r^{\prime}}^{\prime}=\sum_{r^{\prime} \geq s_{i}(b)} F_{i}^{r^{\prime}} z_{r^{\prime}}^{\prime \prime}
$$

and such that $z_{s_{i}(b)}^{\prime \prime} v^{f} \in \mathbf{B}$ for some integer $f$.

In particular, we must have

(c) $b \in F_{i}^{s_{i}(b)} \mathbf{U}^{-}$.

Theorem 11.7. (a) For any $i \in I$ and any $r \geq 0$, the intersection $F_{i}^{r} \mathbf{U}^{-} \cap \mathbf{B}$ is an $A^{\prime}$-basis of $F_{i}^{r} \mathbf{U}^{-}$.

(b) For any $i \in I$ and any $r \geq 0$, the intersection $\mathbf{U}^{-} F_{i}^{r} \cap \mathbf{B}$ is an $A^{\prime}$-basis of $\mathbf{U}^{-} F_{i}^{r}$.

Let $z \in F_{i}^{r} \mathbf{U}^{-}$; we can write uniquely $z=\sum_{b \in \mathbf{B}} f_{b} b$ with $f_{b} \in A^{\prime}$. Assume that $b$ satisfies $f_{b} \neq 0$.

By definition (11.6) we see that $r \leq s_{i}(b)$. By 11.4(c), we have $b \in F_{i}^{s_{i}(b)} \mathbf{U}^{-}$ and in particular, $b \in F_{i}^{r} \mathbf{U}^{-}$. This proves (a). Now (b) follows from (a) and 11.4 .

Corollary 11.8. (a) For any $\nu \in \mathbf{N}^{I}$, the intersection $\left(\sum_{i \in I} F_{i}^{\nu(i)} \mathbf{U}^{-}\right) \cap \mathbf{B}$ is an $\mathbf{A}^{\prime}$-basis of $\sum_{i \in I} F_{i}^{\nu(i)} \mathbf{U}^{-}$.

(b) For any $\nu \in \mathbf{N}^{I}$, the intersection $\left(\sum_{i \in I} \mathbf{U}^{-} F_{i}^{\nu(i)}\right) \cap \mathbf{B}$ is an $\mathbf{A}^{\prime}$-basis of $\sum_{i \in I} \mathbf{U}^{-} F_{i}^{\nu(i)}$. 
11.9. Let $\nu \in \mathbf{N}^{N}$. There exists a $\mathbf{U}$-module $M(\nu)$ with a nonzero vector $y_{0} \in M(\nu)$ with the following properties:

(a) $E_{i} y_{0}=0$ and $K_{i} y_{0}=v^{\nu(i)} y_{0}$ for all $i\left(E_{i}, K_{i}\right.$ are as in 10.15);

(b) the map $\mathbf{U}^{-} \rightarrow M(\nu)$ given by $z \rightarrow z y_{0}$ is surjective and its kernel is exactly $\sum_{i \in I} \mathbf{U}^{-} F_{i}^{\nu(i)+1}$.

Moreover, the pair $\left(M(\nu), y_{0}\right)$ is unique up to a unique isomorphism.

Now using 11.8, we obtain the following result.

Corollary 11.10. The image of $\mathbf{B}-\left(\left(\sum_{i \in I} \mathbf{U}^{-} F_{i}^{\nu(i)+1}\right) \cap \mathbf{B}\right)$ under the map 11.9(b) is a (canonical) $A^{\prime}$-basis of $M(\nu)$.

\section{THE VARIETY $\Lambda_{\mathrm{V}}$}

12.1. We shall fix a function $\varepsilon: H \rightarrow \mathbf{k}^{*}$ such that $\varepsilon(h)+\varepsilon(\bar{h})=0$ for all $h \in H$.

Let $\mathbf{V} \in \mathscr{V}$. The Lie algebra of $G_{\mathbf{v}}$ is $\mathbf{g l}_{\mathbf{v}}=\bigoplus_{i} \operatorname{End}\left(\mathbf{V}_{i}\right)$; it acts on $\mathbf{E}_{\mathbf{v}}$ by $(a, x) \rightarrow[a, x]=x^{\prime}$ where $x_{h}^{\prime}=a_{h^{\prime \prime}} x_{h}-x_{h} a_{h^{\prime}}$ for all $h$.

We define a nondegenerate symplectic form $\langle$,$\rangle on \mathbf{E}_{\mathbf{v}}$ with values in $\mathbf{k}$ by

$$
\langle x, y\rangle=\sum_{h \in H} \varepsilon(h) \operatorname{tr}\left(x_{h} y_{\bar{h}}\right) .
$$

(Here, tr means trace as endomorphism of $\mathbf{V}_{h^{\prime \prime}}$.) This form is clearly $G_{\mathbf{v}^{-}}$ invariant.

The moment map attached to the $G_{\mathbf{v}}$-action on the symplectic vector space $\mathbf{E}_{\mathbf{v}}$ is the map $\psi: \mathbf{E}_{\mathbf{v}} \rightarrow \mathbf{g l}_{\mathbf{v}}$ whose $i$-component $\psi_{i}: \mathbf{E}_{\mathbf{v}} \rightarrow$ End $\mathbf{V}_{i}$ is given by

$$
\psi_{i}(x)=\sum_{h \in H: h^{\prime \prime}=i} \varepsilon(h) x_{h} x_{\bar{h}} .
$$

We have the following identity:

$$
\left\langle[a, x], x^{\prime}\right\rangle=-\left\langle x,\left[a, x^{\prime}\right]\right\rangle=\sum_{i} \operatorname{tr}\left(a_{i}\left(\psi_{i}\left(x+x^{\prime}\right)-\psi_{i}(x)-\psi_{i}\left(x^{\prime}\right)\right)\right)
$$

for all $a \in \mathbf{g l}_{\mathbf{v}}, x, x^{\prime} \in \mathbf{E}_{\mathbf{v}}$.

Definition. $\Lambda_{\mathbf{V}}$ is the set of all nilpotent elements $x \in \mathbf{E}_{\mathbf{v}}$ such that $\psi_{i}(x)=0$ for all $i \in I$.

Clearly, $\Lambda_{\mathbf{v}}$ is a $G_{\mathbf{v}}$-stable, closed subvariety of $\mathbf{E}_{\mathbf{v}}$.

Note that the equations $\psi_{i}(x)=0$ appear in the work [K] of Kronheimer for a very particular $\mathbf{V}$ associated to an extended Dynkin graph (with $\mathbf{V}_{i}$ being the spaces of the irreducible representation of the corresponding finite subgroup of $\left.S L_{2}(\mathbf{C})\right)$. As Ringel informed me, the equations $\psi_{i}(x)=0$ have first appeared (for ordinary Dynkin graphs) in work of Gelfand and Ponomarev around 1979. 
12.2. For any $k \in I$ and $p \in \mathbf{N}$ we consider

$$
\Lambda_{\mathbf{V}, k, p}=\left\{\left(f_{h}\right) \in \Lambda_{\mathbf{V}} \mid \operatorname{codim}_{\mathbf{v}_{k}}\left(\sum_{h \in H: h^{\prime \prime}=k} \operatorname{im}\left(f_{h}: \mathbf{V}_{h^{\prime}} \rightarrow \mathbf{V}_{k}\right)\right)=p\right\} .
$$

It is clear that for fixed $k \in I$ and $p_{0} \geq 0$, the union $\bigcup_{p: p \leq p_{0}} \Lambda_{\mathbf{V}, k, p}$ is an open subvariety of $\Lambda_{\mathbf{V}}$; hence, $\Lambda_{\mathbf{V}, k, p}$ is a locally closed subvariety of $\Lambda_{\mathbf{V}}$. It is clearly $G_{\mathbf{V}}$-stable.

Theorem 12.3. (a) $\Lambda_{\mathbf{V}}$ is a closed subvariety of $\mathbf{E}_{\mathbf{V}}$ of pure dimension $\operatorname{dim} \mathbf{E}_{\mathbf{V}} / 2$ (i.e. each irreducible component of $\Lambda_{\mathbf{V}}$ has dimension equal to $\operatorname{dim} \mathbf{E}_{\mathbf{V}} / 2$ ).

(b) $\Lambda_{\mathbf{V}, k, p}$ has pure dimension $\operatorname{dim} \mathbf{E}_{\mathbf{V}} / 2$.

12.4. Let $k \in I$ and $p \in \mathbf{N}$ be such that $0<p \leq \operatorname{dim} \mathbf{V}_{k}$. Let $V$ be a subspace of $\mathbf{V}_{k}$ of codimension $p$.

Let $\mathbf{V}^{\prime} \in \mathscr{V}$ be defined by $\mathbf{V}_{i}^{\prime}=\mathbf{V}_{i}$ for $i \neq k$ and $\mathbf{V}_{k}^{\prime}=V$.

Let $J=\bigoplus_{i} \operatorname{Hom}\left(\mathbf{V}_{i}^{\prime}, \mathbf{V}_{i}\right)$ and let $J_{0}=\left\{\left(r_{i}\right) \in J \mid r_{i}\right.$ injective for all $\left.i\right\}$. Let $Y$ be the variety of all triples $(t, s, r) \in \Lambda_{\mathbf{V}^{\prime}, k, 0} \times \mathbf{E}_{\mathbf{V}} \times J_{0}$ such that $s_{h} r_{h^{\prime}}=r_{h^{\prime \prime}} t_{h}$ for all $h \in H$ and

$$
\sum_{h \in H: h^{\prime \prime}=k} \varepsilon(h) s_{h} s_{\bar{h}}=0 .
$$

On $Y$ we have a free $G_{\mathbf{V}^{\prime}}$-action

$$
\left(g_{i}\right):\left(\left(t_{h}\right),\left(s_{h}\right),\left(r_{i}\right)\right) \rightarrow\left(\left(g_{h^{\prime \prime}} t_{h} g_{h^{\prime}}^{-1}\right),\left(s_{h}\right),\left(r_{i} g_{i}^{-1}\right)\right) .
$$

Lemma 12.5. (a) The map $(t, s, r) \rightarrow(t, r)$ is a locally trivial fibration $p^{\prime}: Y \rightarrow$ $\Lambda_{\mathbf{V}^{\prime}, k, 0} \times J_{0}$ with fibres isomorphic to $\mathbf{k}^{m}$, where $m=-p\left(\operatorname{dim} \mathbf{V}_{k}-p\right)+$ $p \sum_{h \in H: h^{\prime}=k} \operatorname{dim} \mathbf{V}_{h^{\prime \prime}}$.

(b) The map $(t, s, r) \rightarrow s$ has image equal to $\Lambda_{\mathbf{V}, k, p}$ and it defines a morphism $p^{\prime \prime}: Y \rightarrow \Lambda_{\mathbf{V}, k, p}$ that is a principal $G_{\mathbf{V}^{\prime}}$-bundle.

(c) If $Z^{\prime}$ is an irreducible component of $\Lambda_{\mathbf{V}^{\prime}, k, 0}$, then $Z=p^{\prime \prime}\left(p^{\prime-1}\left(Z^{\prime} \times J_{0}\right)\right)$ is an irreducible component of $\Lambda_{\mathbf{v}, k, p}$.

(d) We have $\operatorname{dim} Z=\operatorname{dim} Z^{\prime}+\left(\operatorname{dim} \mathbf{E}_{\mathbf{v}}-\operatorname{dim} \mathbf{E}_{\mathbf{v}^{\prime}}\right) / 2$.

(e) We have a 1-1 correspondence $Z^{\prime} \leftrightarrow Z$ between the set of irreducible components of $\Lambda_{\mathbf{V}^{\prime}, k, 0}$ and the set of irreducible components of $\Lambda_{\mathbf{V}, k, p}$.

(Compare [L3, 8.5].) Assume that $(t, r) \in \Lambda_{\mathbf{V}^{\prime}, k, 0} \times J_{0}$ has been fixed; let $\mathscr{F}$ be the set of all $s \in \mathbf{E}_{\mathbf{V}}$ such that $(t, s, r) \in Y$. We must show that $\mathscr{F} \cong \mathbf{k}^{m}$.

Choose a $p$-dimensional subspace $\dot{V}$ of $\mathbf{V}_{k}$, complementary to $V$.

Let $\mathscr{F}^{\prime}$ be the kernel of the linear map

$$
\bigoplus_{h: h^{\prime}=k} \operatorname{Hom}\left(\dot{V}, \mathbf{v}_{h^{\prime \prime}}\right) \rightarrow \operatorname{Hom}(\dot{V}, V)
$$

given by $\left(f_{h}\right) \rightarrow \sum_{h \in H: h^{\prime}=k} \varepsilon(\bar{h}) t_{\bar{h}} f_{h}$. It is clear that $s \rightarrow\left(s_{h} \mid \dot{V}\right)$ gives an isomorphism $\mathscr{F} \cong \mathscr{F}^{\prime}$. But the last linear map is surjective since $t \in \Lambda_{\mathbf{v}^{\prime}, k, 0}$; hence its kernel has dimension $m$, as required. Now (b) is easily verified and 
(c), (e) follow immediately from (a), (b). We see also that in (d) we have $\operatorname{dim} Z+\operatorname{dim} G_{\mathbf{V}^{\prime}}=\operatorname{dim} Z^{\prime}+\operatorname{dim} J_{0}+m$. It is clear that $\operatorname{dim} J_{0}-\operatorname{dim} G_{\mathbf{v}^{\prime}}+m=$ $p\left(\operatorname{dim} \mathbf{V}_{k}-p\right)+m=p \sum_{h: h^{\prime}=k} \operatorname{dim} \mathbf{V}_{h^{\prime \prime}}$.

Thus, $\operatorname{dim} Z-\operatorname{dim} Z^{\prime}=p \sum_{h: h^{\prime}=k} \operatorname{dim} \mathbf{V}_{h^{\prime \prime}}$. On the other hand, we have $\operatorname{dim} \mathbf{E}_{\mathbf{v}}-\operatorname{dim} \mathbf{E}_{\mathbf{v}^{\prime}}=2 p \sum_{h: h^{\prime}=k} d_{h^{\prime \prime}}$ and (d) follows.

Lemma 12.6. If $\mathbf{V} \neq 0$, then $\Lambda_{\mathbf{V}}=\bigcup_{k, p>0} \Lambda_{\mathbf{V}, k, p}$.

Let $x \in \Lambda_{\mathbf{v}}$. Then $x$ is nilpotent, and hence by 1.8(b) there exists an $x$ stable flag $\phi=\left(\mathbf{V}=\mathbf{V}^{0} \supset \mathbf{V}^{1} \supset \cdots \supset \mathbf{V}^{m}=0\right)$. Since $\mathbf{V} \neq 0$, we may assume that $\mathbf{V}^{1} \neq \mathbf{V}$. By the definition of a flag (1.4), there is a $k \in I$ such that $\mathbf{V}_{k}^{1} \neq \mathbf{V}_{k}$ and $\mathbf{V}_{j}^{1}=\mathbf{V}_{j}$ for all $j \neq k$. Let $p>0$ be the codimension of $\mathbf{V}_{k}^{1}$ in $\mathbf{V}_{k}$. Let $h \in H$ be such that $h^{\prime \prime}=k$ Then by $1.8(\mathbf{a})$, we have $x_{h}\left(\mathbf{V}_{h^{\prime}}\right) \subset \mathbf{V}_{k}^{1}$ for all $h \in H$ such that $h^{\prime \prime}=k$. Thus, we have $\sum_{h \in H: h^{\prime \prime}=k} x_{h}\left(\mathbf{V}_{h^{\prime}}\right) \subset \mathbf{V}_{k}^{1}$ so that $x \in \Lambda_{\mathbf{V}, k, p}$.

12.7. We now prove Theorem 12.3. (Compare [L3, 8.7].) We may assume that $\mathbf{V} \neq 0$ and that the theorem is already proved for $I$-graded vector spaces of strictly smaller dimension than that of $\mathbf{V}$. From 12.5 and the induction hypothesis we see that $\Lambda_{\mathbf{v}, k, p}$ has pure dimension equal to $\operatorname{dim} \mathbf{E}_{\mathbf{v}} / 2$ whenever $p>0$. Now using 12.6, we see that $\Lambda_{\mathbf{v}}$ has pure dimension equal to $\operatorname{dim} \mathbf{E}_{\mathbf{V}} / 2$. Finally, from this it follows that $\Lambda_{\mathbf{V}, k, 0}$ (which is open in $\Lambda_{\mathbf{V}}$ ) has pure dimension equal to $\operatorname{dim} \mathbf{E}_{\mathrm{V}} / 2$. Theorem 12.3 is proved.

12.8. Let $\mathbf{V} \in \mathscr{V}$ and let $\Omega$ be an orientation for our graph. Note that $\mathbf{E}_{\mathbf{V}, \Omega}$ is a lagrangian subspace of $\mathbf{E}_{\mathbf{v}}$ complementary to the lagrangian subspace $\mathbf{E}_{\mathbf{v}}, \bar{\Omega}$. Hence the symplectic form $\langle$,$\rangle defines a nonsingular pairing \mathbf{E}_{\mathbf{v}, \Omega} \times \mathbf{E}_{\mathbf{v}, \bar{\Omega}} \rightarrow$ k.

This shows in particular that $\mathbf{E}_{\mathbf{v}}$ is naturally the cotangent bundle of $\mathbf{E}_{\mathbf{V}, \Omega}$. We have the following result (compare [L3, 9.3]):

(a) If $x^{\prime} \in \mathbf{E}_{\mathbf{v}, \Omega}$ and $x^{\prime \prime} \in \mathbf{E}_{\mathbf{v}, \bar{\Omega}}$ then we have $\psi_{i}\left(x^{\prime}+x^{\prime \prime}\right)=0$ for all $i \in I$ if and only if $x^{\prime \prime}$ is orthogonal with respect to $\langle$,$\rangle to the tangent space to$ the $G_{\mathbf{v}}$-orbit of $x^{\prime}$ (regarded as a vector subspace of $\mathbf{E}_{\mathbf{v}, \Omega}$ ).

Indeed, that tangent space is the set of all vectors $x \in \mathbf{E}_{\mathbf{v}, \Omega}$ such that for some $a \in \mathbf{g l}_{\mathbf{y}}$ we have $x=\left[a, x^{\prime}\right]$. The orthogonal to that tangent space is the set of all $x^{\prime \prime} \in \mathbf{E}_{\mathbf{V}, \bar{\Omega}}$ such that for all $a \in \mathbf{g l}_{\mathbf{v}}$ we have $\left\langle\left[a, x^{\prime}\right], x^{\prime \prime}\right\rangle=$ 0 , or equivalently (see $12.1(\mathrm{a})) \sum_{i \in I} \operatorname{tr}\left(a_{i} \psi_{i}\left(x^{\prime}+x^{\prime \prime}\right)\right)=0$, or equivalently $\psi_{i}\left(x^{\prime}+x^{\prime \prime}\right)=0$ for all $i$.

Theorem 12.9. $\Lambda_{\mathbf{V}}$ is a Lagrangian subvariety of $\mathbf{E}_{\mathbf{V}}$.

The proof is based on the inductive construction of irreducible components of $\Lambda_{\mathrm{v}}$ which has been already used in the proof of 12.3; the details will be given elsewhere. This result is not used in the sequel.

12.10. Let $\widetilde{M}(\mathbf{V})$ be the $\mathbf{Q}$-vector space consisting of all constructible functions in $M\left(\Lambda_{\mathbf{V}}\right)$ that are constant on the orbits of $G_{\mathbf{V}}$ on $\Lambda_{\mathbf{V}}$. 
Now let $\mathbf{V}, \mathbf{V}^{\prime}, \mathbf{V}^{\prime \prime}$ be as in 3.1, and let $f^{\prime} \in \widetilde{M}\left(\mathbf{V}^{\prime}\right), f^{\prime \prime} \in \widetilde{M}\left(\mathbf{V}^{\prime \prime}\right)$.

We define a function $f^{\prime} * f^{\prime \prime} \in \widetilde{M}(\mathbf{V})$ by imitating the construction of 3.1. Consider the diagram (analogous to 3.1(a)):

$$
\Lambda_{\mathbf{V}^{\prime}} \times \Lambda_{\mathbf{V}^{\prime \prime}} \stackrel{p_{1}}{\longleftarrow} \mathbf{F}^{\prime} \stackrel{p_{2}}{\longrightarrow} \mathbf{F}^{\prime \prime} \stackrel{p_{3}}{\longrightarrow} \Lambda_{\mathbf{V}}
$$

where the notations are as follows.

$\mathbf{F}^{\prime \prime}$ is the variety of all pairs $(x, V)$ where $x \in \Lambda_{\mathbf{v}}$ and $V$ is an $x$-stable $I$-graded subspace of $\mathbf{V}$ such that $V \in \mathscr{V}_{\nu^{\prime \prime}}$.

$\mathbf{F}^{\prime}$ is the variety of all quadruples $\left(x, V, R^{\prime \prime}, R^{\prime}\right)$ where $(x, V) \in \mathbf{F}^{\prime \prime}, R^{\prime \prime}$ is an isomorphism $\mathbf{V}^{\prime \prime} \cong V$ (in $\mathscr{V}$ ) and $R^{\prime}$ is an isomorphism $\mathbf{V}^{\prime} \cong \mathbf{V} / V$ (in $\mathscr{V})$.

We have $p_{1}\left(x, V, R^{\prime \prime}, R^{\prime}\right)=\left(x^{\prime}, x^{\prime \prime}\right)$ where $x_{h} R_{h^{\prime}}^{\prime}=R_{h^{\prime \prime}}^{\prime} x_{h}^{\prime}: \mathbf{V}_{h^{\prime}}^{\prime} \rightarrow \mathbf{V}_{h^{\prime \prime}} / V_{h^{\prime}}$ and $x_{h} R_{h^{\prime}}^{\prime \prime}=R_{h^{\prime \prime}}^{\prime \prime} x_{h}^{\prime \prime}: \mathbf{V}_{h^{\prime}}^{\prime \prime} \rightarrow V_{h^{\prime \prime}}$ for all $h \in H$.

$p_{2}\left(x, V, R^{\prime \prime}, R^{\prime}\right)=(x, V), p_{3}(x, V)=x$.

Note that $p_{2}$ is a $G_{\mathbf{V}^{\prime}} \times G_{\mathbf{V}^{\prime \prime}}$-principal bundle and $p_{3}$ is proper. (Unlike the situation in 3.1, $p_{1}$ is not in general smooth.)

Let $f_{1} \in M\left(\Lambda_{\mathbf{v}^{\prime}} \times \Lambda_{\mathbf{v}^{\prime \prime}, \Omega}\right)$ be given by $f_{1}\left(x^{\prime}, x^{\prime \prime}\right)=f^{\prime}\left(x^{\prime}\right) f^{\prime \prime}\left(x^{\prime \prime}\right)$. Then there is a unique function $f_{3} \in M\left(\mathbf{F}^{\prime \prime}\right)$ such that $p_{1}^{*} f_{1}=p_{3}^{*} f_{3}$; by definition, $f^{\prime} * f^{\prime \prime}=\left(p_{3}\right)_{!}\left(f_{3}\right)$.

Next we note that given $\nu \in \mathbf{N}^{I}$, the vector spaces $\widetilde{M}(\mathbf{V})$ for various $\mathbf{V} \in \mathscr{V}_{\nu}$ can all be identified in a coherent way with a single vector space $\widetilde{M}(\nu)$ (by the invariance condition on the functions considered). The operation above becomes a pairing $\widetilde{M}\left(\nu^{\prime}\right) \times \widetilde{M}\left(\nu^{\prime \prime}\right) \rightarrow \widetilde{M}(\nu)$ denoted $\left(f^{\prime}, f^{\prime \prime}\right) \rightarrow f^{\prime} * f^{\prime \prime}$. Let $\widetilde{M}=\bigoplus_{\nu} \widetilde{M}(\nu)$. The operation $*$ makes $\widetilde{M}$ into an associative Q-algebra. (It is a quotient of an algebra like $\mathscr{M}(\Omega)$ in 10.18 defined for a graph with the same vertices as our graph, but with twice as many edges, in which $H$ is an orientation.)

Let $i \in I$; let $\mu$ be such that $\mu(i)=1$ and $\mu(j)=0$ for $j \neq i$. Then $\widetilde{M}(\mu)$ is one dimensional, with a canonical basis element $F_{i}$ corresponding to the function on $\{0\}$ with value 1 . Let $\widetilde{M}_{0}$ be the subalgebra of $\widetilde{M}$ generated by the elements $F_{i} \quad(i \in I)$. Then $\widetilde{M}_{0}=\bigoplus_{\nu} \widetilde{M}_{0}(\nu)$ where $\widetilde{M}_{0}(\nu)=\widetilde{M}(\nu) \cap \widetilde{M}_{0}$.

Lemma 12.11. Let $i \neq j$ in $I$ and let $N$ be the number of edges in our graph that join $i, j$. Then the following identity holds in the algebra $\widetilde{M}_{0}$ :

$$
\sum_{p=0}^{N+1}(-1)^{p} \frac{F_{i}^{p}}{p !} F_{j} \frac{F_{i}^{N+1-p}}{(N+1-p) !}=0
$$

Let $\mathbf{V} \in \mathscr{V}$ be such that $\operatorname{dim} \mathbf{V}_{i}=1, \operatorname{dim} \mathbf{V}_{j}=N+1$, and $\operatorname{dim} \mathbf{V}_{k}=0$ for all other $k$. Let $H^{\prime}=\left\{h \in H \mid h^{\prime}=i, h^{\prime \prime}=j\right\}$.

In our case,

$$
\Lambda_{\mathbf{v}}=\left\{x \in \mathbf{E}_{\mathbf{v}} \mid \sum_{h \in H^{\prime}} \varepsilon(h) x_{h} x_{\bar{h}}=0 \text { and } x_{\bar{h}_{1}} x_{h_{2}}=0 \text { for all } h_{1}, h_{2} \in H^{\prime}\right\} .
$$


(The first condition is $\psi_{j}(x)=0$; the second one is the nilpotency condition. The condition $\psi_{i}(x)=0$ is a consequence of the nilpotency condition.)

Let $x \in \Lambda_{\mathbf{v}}$. Let $W_{1}$ be the subspace of $\mathbf{V}_{j}$ generated by the images of $x_{h}: \mathbf{V}_{i} \rightarrow \mathbf{V}_{j}$ for various $h \in H^{\prime}$. Let $W_{2}$ be the subspace of $\mathbf{V}_{j}$ given by the intersection of the kernels of $x_{\bar{h}}: \mathbf{V}_{j} \rightarrow \mathbf{V}_{i}$ for various $h \in H^{\prime}$. From (a) we have $W_{1} \subset W_{2}$.

For each $p \in[0, N+1]$, let $\mathbf{F}_{p}(x)$ be the variety of all codimension $p$ subspaces $W$ of $\mathbf{v}_{j}$ such that $W_{1} \subset W \subset W_{2}$.

The identity to be proved can be rewritten in the following form:

$$
\sum_{p=0}^{N+1}(-1)^{p} \chi\left(\mathbf{F}_{p}(x)\right)=0
$$

for all $x \in \Lambda_{\mathbf{v}}$. Let $d_{1}, d_{2}$ be the codimension of $W_{1}, W_{2}$, respectively.

Now $\mathbf{F}_{p}(x)$ is empty unless $d_{2} \leq p \leq d_{1}$, in which case it is a Grassmannian with Euler characteristic $\chi\left(\mathbf{F}_{p}(x)\right)=\left(\begin{array}{c}d_{1}-d_{2} \\ d_{1}-p\end{array}\right)$. If $d_{1}>d_{2}$, we have the identity

$$
\sum_{p=d_{2}}^{d_{1}}(-1)^{p}\left(\begin{array}{c}
d_{1}-d_{2} \\
d_{1}-p
\end{array}\right)=0
$$

and (b) follows.

It remains to show that we always have $W_{1} \neq W_{2}$. We have a diagram

$$
O \rightarrow \mathbf{V}_{j} / W_{2} \rightarrow \mathbf{V}_{i} \oplus \cdots \oplus \mathbf{v}_{i} \rightarrow W_{1} \rightarrow 0
$$

where the middle term is a direct sum of copies of $\mathbf{V}_{1}$, one for each $h \in$ $H^{\prime}$; the second arrow is defined by $y \rightarrow\left(x_{\bar{h}}(y)\right)$; the third arrow is $\left(z_{h}\right) \rightarrow$ $\sum_{h \in H^{\prime}} \varepsilon(h) x_{h}\left(z_{h}\right)$. This diagram is a complex (by (a)), which is acyclic except possibly at the middle position. It follows that $\operatorname{dim}\left(\mathbf{V}_{j} / W_{2}\right) \leq N-\operatorname{dim} W_{1}$ so that $\operatorname{dim} W_{2}-\operatorname{dim} W_{1} \geq \operatorname{dim} \mathbf{V}_{j}-N=1$; in particular, we have $W_{1} \neq W_{2}$ and the lemma is proved.

12.12. From 12.11 we see that there is a unique homomorphism of $\mathbf{Q}$-algebras $\gamma: u^{-} \rightarrow \widetilde{M}_{0}$ that takes $F_{i}$ to $F_{i}$ for each $i \in I .\left(u^{-}\right.$is as in 10.15.)

The following result provides a description of the algebra $u^{-}$parallel to that in 10.18, but without reference to any orientation of our graph.

Theorem 12.13. $\gamma$ is an algebra isomorphism $u^{-} \cong \widetilde{M}_{0}$.

By the definition of $u^{-}$and $\widetilde{M}_{0}$, we have that $\gamma$ is surjective. To prove that $\gamma$ is injective, we choose, as we may, an orientation $\Omega$ for our graph that has no cycles (see 10.20).

We define an algebra homomorphism $\widetilde{M} \rightarrow \mathscr{M}(\Omega)$ as follows. Let $\mathrm{V} \in$ $\mathscr{V}$. Then $\mathbf{E}_{\mathbf{V}, \Omega}$ is naturally a subspace of $\Lambda_{\mathbf{V}}$. (Any element of $\mathbf{E}_{\mathbf{V}, \Omega}$ is nilpotent since $\Omega$ has no cycles; any element $x \in \mathbf{E}_{\mathbf{V}, \Omega}$ satisfies automatically the equations $\psi(x)=0$ by the definition of an orientation.) Hence restriction 
of functions defines a linear map $\widetilde{M}(\mathbf{V}) \rightarrow \mathscr{M}(\mathbf{V}, \Omega)$. It is obvious that this is compatible with the operations $*$. These maps then define the required algebra homomorphism $\widetilde{M} \rightarrow \mathscr{M}(\Omega)$.

Consider the composition of homomorphisms

$$
u^{-} \rightarrow \widetilde{M}_{0} \rightarrow \widetilde{M} \rightarrow \mathscr{M}(\Omega)
$$

where the first one is $\gamma$, the second one is the inclusion, and the third one has been just constructed. This composition coincides with the homomorphism

$$
u^{-} \cong \mathscr{M}_{0}^{\prime}(\Omega)=\mathscr{M}_{0}(\Omega) \subset \mathscr{M}(\Omega)
$$

(of $10.20,10.19(\mathrm{a})$ ). (It is enough to check that these coincide on the generators $F_{i}$, which is obvious.) Hence the kernel of $\gamma$ is contained in the kernel of the homomorphism 10.20. The last kernel is zero, and hence so is the kernel of $\gamma$. The theorem is proved.

12.14. Let $X$ be an irreducible component of $\Lambda_{\mathbf{V}}$ where $\mathbf{V} \in \mathscr{V}_{\nu}$. We define a linear form $T_{X}: \widetilde{M}_{0}(\nu) \rightarrow \mathbf{Q}$ as follows. Under the canonical identification $\widetilde{M}(\mathbf{V}) \cong \widetilde{M}(\nu)$, the subspace $\widetilde{M}_{0}(\nu)$ of $\widetilde{M}(\nu)$ is identified to a subspace $\widetilde{M}_{0}(\mathbf{V})$ of $\widetilde{M}(\mathbf{V})$. We can find an open dense subset $X_{0}$ of $X$ such that any function $f \in \widetilde{M}_{0}(\mathbf{V})$ is constant on $X_{0}$. (Such an open set exists separately for each $f$ since $f$ is constructible; one exists for all $f$ simultaneously since the vector space $\widetilde{M}_{0}(\mathbf{V})$ is finite dimensional.) We then define $T_{X}(f)$ to be the (constant) value of $f$ on $X_{0}$.

Let us denote by $Z_{\nu}$ the set of irreducible components of $\Lambda_{\mathbf{v}}$. This set is independent of the choice of $\mathbf{V}$ as long as $\mathbf{V} \in \mathscr{V}_{\nu}$.

By associating to each $f \in \widetilde{M}_{0}(\nu)$ the function $X \rightarrow T_{X}(f)$, we thus obtain a linear function from $\widetilde{M}_{0}(\nu)$ to the vector space of all functions $Z_{\nu} \rightarrow \mathbf{Q}$.

It may be conjectured that this is an isomorphism. This would imply that the $\nu$-homogeneous part of $u^{-}$has dimension equal to the number of elements in $Z_{\nu}$. (This last fact is actually true for graphs of type $A, D, E$ or of affine type $A$, as can be seen from 14.2 and 15.6.)

12.15. In this section we will show that the constructions in this section are essentially independent of the choice of the function $\varepsilon$. Let $\varepsilon^{\prime}: H \rightarrow \mathbf{k}^{*}$ be another function such that $\varepsilon^{\prime}(h)+\varepsilon^{\prime}(\bar{h})=0$ for all $h \in H$. Let $\langle,\rangle^{\prime}, \psi_{i}^{\prime}$, $\Lambda_{\mathbf{V}}^{\prime}$ be defined as $\langle\rangle,, \psi_{i}, \Lambda_{\mathbf{v}}$ but in terms of $\varepsilon^{\prime}$ instead of $\varepsilon$. We can find a function $\delta: H \rightarrow \mathbf{k}^{*}$ such that $\delta(h)=\delta(\bar{h}), \delta(h)^{2}=\varepsilon^{\prime}(h) \varepsilon(h)^{-1}$ for all $h \in H$.

Then the linear map $A: \mathbf{E}_{\mathbf{v}} \rightarrow \mathbf{E}_{\mathbf{v}}$ given by $A(x)=x^{\prime}, x_{h}^{\prime}=\delta(h) x_{h}$ for all $h \in H$ is an isomorphism such that $\langle A(x), A(y)\rangle=\langle x, y\rangle^{\prime}$ for all $x, y \in \mathbf{E}_{\mathbf{v}}$, $\psi_{i}(A(x))=\psi_{i}^{\prime}(x)$ for all $x \in \mathbf{E}_{\mathbf{V}}$, and $A\left(\Lambda_{\mathbf{V}}^{\prime}\right)=\Lambda_{\mathbf{V}}$.

Moreover, the isomorphism $A: \Lambda_{\mathrm{V}}^{\prime} \rightarrow \Lambda_{\mathrm{V}}$ induces a bijection on the sets of irreducible components that is independent of the choice of $\delta$. To prove this, we may assume that $\varepsilon^{\prime}=\varepsilon$ so that $\delta(h)=\delta(\bar{h})= \pm 1$. We can find 
$m: H \rightarrow \mathbf{Z}$ such that $m(h)+m(\bar{h})=0,(-1)^{m(h)}=\delta(h)$ for all $h \in H$. Define $A_{t}: \mathbf{E}_{\mathbf{V}} \rightarrow \mathbf{E}_{\mathbf{v}}$ for all $t \in \mathbf{k}^{*}$ by $A_{t}(x)=x^{\prime}, x_{h}^{\prime}=t^{m(h)} x_{h}$. Then $A_{t}$ defines a 1-parameter group of automorphisms of $\Lambda_{\mathrm{V}}$; hence it induces the identity map on the set of irreducible components of $\Lambda_{\mathrm{V}}$. We have $A_{-1}=A$ and our assertion follows.

Clearly, we can always choose $\varepsilon$ so that $\varepsilon(h)= \pm 1$ for all $h \in H$.

\section{Singular SUPPORTS}

13.1. In this section we assume that $\mathbf{k}$ has characteristic zero.

Let $\pi: X \rightarrow Y$ be a proper morphism between smooth, connected varieties. Let $S=\pi(X)$, a closed irreducible subvariety of $Y$.

Let $\tilde{X}$ be the variety of all pairs $(x, \xi)$ where $x \in X$ and $\xi \in T_{\pi(x)}^{*} Y$ (cotangent space of $\mathrm{Y}$ at $\pi(x)$ ) are such that $\xi$ is in the kernel of the canonical map $T_{\pi(x)}^{*} Y \rightarrow T_{x}^{*} X$ induced by $\pi$. The map $\tilde{X} \rightarrow T^{*} Y$ defined by $(x, \xi) \rightarrow \xi$ is a proper morphism. We denote its image by $\widetilde{Y}$ (a closed subvariety of $T^{*} Y$ ) and we denote $\tilde{\pi}: \widetilde{X} \rightarrow \widetilde{Y}$ the resulting surjective map.

For any $L \in \mathscr{D}(Y)$ we denote by $S S(L)$ the singular support (or characteristic variety ) of $L$. It is known that $S S(L)$ is a closed lagrangian subvariety of $T^{*} Y$. According to [KS],

(a) $S S\left(\pi_{!}(1)\right)$ is contained in $\tilde{Y}$.

13.2. Let $\mathbf{V} \in \mathscr{V}_{\nu}$ and let $(\mathbf{i}, \mathbf{a}) \in S_{\nu}$. We fix an orientation $\Omega$. Let $\pi=$ $\pi_{\mathbf{i}, \mathbf{a}}: \widetilde{\mathscr{F}}_{\mathbf{i}, \mathbf{a}} \rightarrow \mathbf{E}_{\mathbf{V}, \Omega}$ be as in 1.5. By 1.6, the definitions and results of 13.1 are applicable to this $\pi$ (with $X=\widetilde{\mathscr{F}}_{\mathbf{i}, \mathbf{a}}$ and $Y=\mathbf{E}_{\mathbf{V}, \Omega}$ ). In our case, $T^{*} Y$ may be identified with $\mathbf{E}_{\mathbf{V}}$ as in 12.8 . Hence $\tilde{Y}$ may be regarded as a subvariety of $\mathbf{E}_{\mathbf{v}}$. With these notations, we have the following result.

Theorem 13.3. (a) We have

$$
\widetilde{Y}=\left\{z \in \Lambda_{\mathbf{V}} \mid z \text { leaves stable some } \phi \in \mathscr{F}_{\mathbf{i}, \mathbf{a}}\right\} \text {. }
$$

(b) One can identify

$$
\widetilde{X}=\left\{(z, \phi) \in \Lambda_{\mathbf{v}} \times \mathscr{F}_{\mathbf{i}, \mathbf{a}} \mid \phi \text { is } z \text {-stable }\right\}
$$

so that the map $\tilde{\pi}: \tilde{X} \rightarrow \tilde{Y}$ of 13.1 is $(z, \phi) \rightarrow z$.

13.4. We begin with some preliminaries to the proof of 13.3. Let $(x, \phi) \in \widetilde{\mathscr{F}_{\mathrm{i}, \mathrm{a}}}$ where $\phi=\left(\mathbf{V}=\mathbf{V}^{0} \supset \mathbf{V}^{1} \supset \cdots \supset \mathbf{V}^{m}=0\right)$ is as in 1.4. Let $\mathbf{b}_{0}$ be the subspace of $\mathbf{g l}_{\mathbf{V}}$ consisting of all $f \in \mathbf{g l}_{\mathbf{V}}$ such that $f\left(\mathbf{V}^{l}\right) \subset \mathbf{V}^{l}$ for all $l$.

Let $\mathbf{b}_{0}^{0}$ be the subspace of $\mathbf{b}_{0}$ consisting of all $f \in \mathbf{b}_{0}$ that are nilpotent as endomorphisms of $\bigoplus_{i} \mathbf{V}_{i}$.

Let $\mathbf{b}_{+}$be the subspace of $\mathbf{E}_{\mathbf{V}, \Omega}$ consisting of all $y \in \mathbf{E}_{\mathbf{V}, \Omega}$ such that $\phi$ is $y$-stable.

Let $\mathbf{b}_{\text {_ }}$ be the subspace of $\mathbf{E}_{\mathbf{V}, \bar{\Omega}}$ consisting of all $y \in \mathbf{E}_{\mathbf{V}, \bar{\Omega}}$ such that $\phi$ is $y$-stable. 
The tangent space at $(x, \phi)$ to $\widetilde{F}_{\mathbf{i}, \mathbf{a}}$ is naturally the kernel of the linear map (a)

$$
\mathbf{E}_{\mathbf{v}, \Omega} \oplus \mathbf{g l}_{\mathbf{V}} / \mathbf{b}_{0} \rightarrow \mathbf{E}_{\mathbf{V}, \Omega} / \mathbf{b}_{+}
$$

given by $(A, B) \rightarrow C, C_{h}=A_{h}+x_{h} B_{h^{\prime}}-B_{h^{\prime \prime}} x_{h} \quad(h \in H)$.

We now define a linear map

$$
\mathbf{b}_{-} \rightarrow \mathbf{E}_{\mathbf{V}, \bar{\Omega}} \oplus \mathbf{b}_{0}^{0}
$$

by $D \rightarrow(D, E)$ where $E=\left(E_{i}\right)$ is given by

$$
E_{i}=\sum_{h \in H: h^{\prime \prime}=i} \varepsilon(h)\left(-x_{h} D_{\bar{h}}-D_{h} x_{\bar{h}}\right) .
$$

To see that this is well defined, we must check that, in the previous formula, $E_{i}: \mathbf{V}_{i} \rightarrow \mathbf{V}_{i}$ is nilpotent. Now $\phi$ is $x$-stable and $D$-stable hence $E$-stable, so that $E$ is nilpotent by $1.8(\mathrm{a})$.

We now show that $(b)$ is naturally the transpose of $(a)$.

First, $\mathbf{E}_{\mathbf{V}, \bar{\Omega}}$ is naturally the dual space of $\mathbf{E}_{\mathbf{v}, \Omega}$ via the pairing induced by $\langle$,$\rangle .$

The same pairing identifies $\mathbf{b}_{-}$with the dual space of $\mathbf{E}_{\mathbf{v}, \Omega} / \mathbf{b}_{+}$. (It is easy to check that $\operatorname{dim} \mathbf{b}_{-}+\operatorname{dim} \mathbf{b}_{+}=\operatorname{dim} \mathbf{E}_{\mathbf{v}, \Omega}$ and that $\langle x, y\rangle=0$ for all $x \in \mathbf{b}_{+}$, $y \in \mathbf{b}_{-}$. Note that $\langle x, y\rangle=\sum_{i \in I} \operatorname{tr} T_{i}$ where $T_{i}=\sum_{h ; h^{\prime \prime}=i} x_{h} y_{\bar{h}}$. Now $\phi$ is $x$-stable and $y$-stable hence $T$-stable, so that $T_{i}$ is nilpotent by 1.8(a). Thus $\operatorname{tr} T_{i}=0$; hence $\langle x, y\rangle=0$.)

Note also that $\mathbf{b}_{0}^{0}$ is naturally the dual space of $\mathbf{g l}_{\mathbf{v}} / \mathbf{b}_{0}$ via the pairing induced by the pairing $\mathbf{g l}_{\mathbf{v}} \times \mathbf{g l}_{\mathbf{v}} \rightarrow \mathbf{C}, A, A^{\prime} \rightarrow \operatorname{tr}\left(A A^{\prime}\right)$.

It remains to verify the following identity:

$$
\langle A, D\rangle+\operatorname{tr}(B E)=\langle C, D\rangle
$$

for any $A \in \mathbf{E}_{\mathbf{v}, \Omega}, B \in \mathbf{g l}_{\mathbf{v}}, D \in \mathbf{b}_{\text {_ }}$ where $C \in \mathbf{E}_{\mathbf{v}, \Omega}$ is defined in terms of $A, B$ as in (a) and $E \in \mathbf{g l}_{\mathbf{v}}$ is defined in terms of $D$ as in (b). Thus, we must verify the identity:

$$
\begin{gathered}
\sum_{h} \varepsilon(h)\left(\operatorname{tr}\left(A_{h} D_{\bar{h}}\right)-\operatorname{tr}\left(B_{h^{\prime \prime}} x_{h} D_{\bar{h}}\right)-\operatorname{tr}\left(B_{h^{\prime \prime}} D_{h} x_{\bar{h}}\right)\right) \\
=\sum_{h} \varepsilon(h) \operatorname{tr}\left(\left(A_{h}+x_{h} B_{h^{\prime}}-B_{h^{\prime \prime}} x_{h}\right) D_{\bar{h}}\right) .
\end{gathered}
$$

But this is clear.

Thus we have proved that (b) is the transpose of (a). It follows that

(c) the cotangent space at $(x, \phi)$ to $\widetilde{\mathscr{F}}_{\mathbf{i}, \mathbf{a}}$ is naturally the cokernel of the linear map (b).

13.5. We now prove Theorem 13.3. Note that the cotangent space at $x$ to $Y=\mathbf{E}_{\mathbf{V}, \Omega}$ is naturally $\mathbf{E}_{\mathbf{V}, \bar{\Omega}}$ (see 12.8 ) and the linear map $T^{*} \pi$ induced by $\pi$ from the cotangent space at $x$ to $Y=\mathbf{E}_{\mathbf{v}, \Omega}$ to the cotangent space at $(x, \phi)$ to $X=\widetilde{\mathscr{F}_{\mathbf{i}, \mathbf{a}}}$ is given by $y \rightarrow T^{*} \pi(y)=(y, 0)$ modulo the image of $13.4(\mathrm{~b})$. 
The condition that $y \in \mathbf{E}_{\mathbf{v}, \bar{\Omega}}$ satisfies $T^{*} \pi(y)=0$ is therefore that $y \in \mathbf{b}_{-}$ and $\sum_{h: h^{\prime \prime}=i} \varepsilon(h)\left(x_{h} y_{\bar{h}}+y_{h} x_{\bar{h}}\right)=0$ for all $i \in I$. Since $x_{h} x_{\bar{h}}=0$ and $y_{h} y_{\bar{h}}=0$ for all $h$ we see that the previous condition is equivalent to

$$
\phi \text { is } y \text {-stable and } \psi_{i}(x+y)=0 \text { for all } i \in I \text {. }
$$

For $y \in \mathbf{E}_{\mathbf{v}, \bar{\Omega}}$, the conditions (a), (b) below are equivalent:

$\phi$ is $y$-stable,

$$
x+y \text { is nilpotent and it leaves } \phi \text { stable. }
$$

Clearly, if (b) holds then (a) holds (since $\phi$ is $x$-stable). If (a) holds then certainly $\phi$ is $(x+y)$-stable and from this we see using 1.8(a) that $x+y$ is nilpotent. Thus the equivalence of $(a),(b)$ is established.

We now see that $y \in \mathbf{E}_{\mathbf{v}, \bar{\Omega}}$ satisfies $T^{*} \pi(y)=0$ if and only if

$$
x+y \in \Lambda_{\mathbf{v}} \text { and } \phi \text { is }(x+y) \text {-stable. }
$$

Theorem 13.3 follows.

Corollary 13.6. For any $L \in \mathscr{P}_{\mathbf{v}, \Omega}$, the singular support $S S(L)$ is a union of irreducible components of $\Lambda_{\mathbf{v}}$.

By definition, we can find $\pi$ as in 13.2 such that some shift of $L$ is isomorphic to a direct summand of some $\pi_{1}(1)$. We therefore have $S S(L) \subset$ $S S\left(\pi_{t}(1)\right)$. By 13.1(a), we have $S S\left(\pi_{t}(1)\right) \subset \widetilde{Y}$ and by $13.3($ a) we have $\widetilde{Y} \subset$ $\Lambda_{\mathrm{v}}$. From these inclusions we deduce that $S S(L) \subset \Lambda_{\mathrm{v}}$. Now $S S(L)$ is a closed lagrangian subvariety of $\mathbf{E}_{\mathbf{v}}$. Hence it has pure dimension equal to $\operatorname{dim}\left(\mathbf{E}_{\mathbf{v}}\right) / 2$. On the other hand, according to $12.3, \Lambda_{\mathbf{V}}$ also has pure dimension equal to $\operatorname{dim}\left(\mathbf{E}_{\mathbf{v}}\right) / 2$. The corollary follows.

13.7. One may hope that the following two statements might be true:

(a) for graphs of type $A, D, E$, the singular support of any $L \in \mathscr{P}_{\mathbf{v}, \Omega}$ is irreducible, and

(b) for general graphs, there is a unique 1-1 correspondence $L \rightarrow X_{L}$ between $\mathscr{P}_{\mathbf{V}, \Omega}$ and the set of irreducible components of $\Lambda_{\mathbf{v}}$ such that $X_{L} \subset S S(L)$.

Statement (a) for type $A$ is closely related to the expected irreducibility of singular supports for Schubert varieties of $G L(n)$.

13.8. We consider an example. We take $I=\{i, j\}, H=\left\{h_{1}, h_{2}, h_{3}, h_{4}\right\}$, with $h_{1}^{\prime}=h_{2}^{\prime}=i, h_{1}^{\prime \prime}=h_{2}^{\prime \prime}=j, \bar{h}_{1}=h_{3}, \bar{h}_{2}=h_{4}$. We take $\Omega=\left\{h_{1}, h_{2}\right\}$, $\varepsilon\left(h_{1}\right)=\varepsilon\left(h_{2}\right)=1, \varepsilon\left(h_{3}\right)=\varepsilon\left(h_{4}\right)=-1$. We take $\mathbf{V}$ such that $\mathbf{V}_{i}=\mathbf{V}_{j}=V$ is a two-dimensional vector space. We identify $\mathbf{E}_{\mathbf{v}}$ with the set of all quadruples $\left(A, B, A^{\prime}, B^{\prime}\right)$ of endomorphisms of $V, \mathbf{E}_{\mathbf{v}, \Omega}$ with the subspace of $\mathbf{E}_{\mathbf{V}}$ defined by $A^{\prime}=B^{\prime}=0$ and $\Lambda_{\mathbf{v}}$ with the subspace of $\mathbf{E}_{\mathbf{v}}$ defined by the equations $A A^{\prime}+B B^{\prime}=0, A^{\prime} A+B^{\prime} B=0$, and by the condition that $A A^{\prime}, A B^{\prime}, B A^{\prime}, B B^{\prime}$ are nilpotent. 
Then $\mathscr{P}_{\mathbf{v}, \Omega}$ consists of 6 perverse sheaves $L_{1}, \ldots, L_{6}$ with supports of dimensions $0,4,5,5,8,8$ respectively. ( $L_{3}, L_{4}$ have different supports but $L_{5}, L_{6}$ have the same support.) The corresponding local system on an open dense part of the support is trivial for $L_{1}, \ldots, L_{5}$ and nontrivial for $L_{6}$.

Now $\Lambda_{\mathbf{v}}$ has six irreducible components $X_{1}, \ldots, X_{6}$. Notations can be arranged so that the following holds: $S S\left(L_{j}\right)=X_{j}$ for $j=1, \ldots, 5$ and $S S\left(L_{6}\right)=X_{5} \cup X_{6}$. Note also that $X_{6}$ is the closure of the conormal bundle of a 7-dimensional irreducible submanifold of $\mathbf{E}_{\mathbf{V}, \Omega}$. Hence 13.7(b) holds in our example.

\section{EXAMPLE: GRAPHS OF TYPE $A, D, E$}

14.1. In this section we assume that our graph is of type $A, D$ or $E$; in particular, there is at most one edge joining two vertices. We fix an orientation $\Omega$ for the graph. Let $\mathbf{V} \in \mathscr{V}$.

Proposition 14.2. (a) Any element $x \in \mathbf{E}_{\mathbf{v}}$ such that $\psi_{i}(x)=0$ for all $i \in I$ is automatically nilpotent.

(b) The irreducible components of $\Lambda_{\mathbf{v}}$ are the closures of the conormal bundles of the various $G_{\mathbf{v}}$-orbits in $\mathbf{E}_{\mathbf{V}, \Omega}$.

Assume that (a) holds. Then, using (a) and 12.8(a) we see that $\Lambda_{\mathrm{V}}$ is precisely the union of the conormal bundles of the various $G_{\mathbf{V}}$-orbits in $\mathbf{E}_{\mathbf{V}, \Omega}$. Since there are only finitely many such orbits (Gabriel's theorem) we see that (b) holds. It remains to prove (a). The proof of (a) has much in common with that in $[\mathrm{L} 2, \S 10]$.

We can write uniquely $x=y+z$ where $y \in \mathbf{E}_{\mathbf{v}, \Omega}$ and $z \in \mathbf{E}_{\mathbf{v}, \bar{\Omega}}$. We may regard $(\mathbf{V}, y)$ as a representation of our oriented graph. Writing this representation as a direct sum of indecomposable representations and using $[\mathrm{L} 2,4.9(\mathrm{c})]$ we see that there exists a direct sum decomposition $\mathbf{V}=\bigoplus_{p=1}^{\nu} \mathbf{V}^{p}$ where each $\mathbf{V}^{p} \in \mathscr{V}$ is $y$-stable (i.e., $\left(\mathbf{V}^{p}, y\right)$ is a representation of our oriented graph) and such that that any morphism from the representation $\left(\mathbf{V}^{p}, y\right)$ to the representation $\left(\mathbf{V}^{p^{\prime}}, y\right)$ is zero whenever $p^{\prime}<p$.

We have a direct sum decomposition

$$
\mathbf{E}_{\mathbf{v}, \Omega}=\bigoplus_{p, p^{\prime} \in[1, \nu]} \mathbf{E}_{\mathbf{v}, \Omega}^{p, p^{\prime}} \quad \text { where } \mathbf{E}_{\mathbf{v}, \Omega}^{p, p^{\prime}}=\bigoplus_{h \in \Omega} \operatorname{Hom}\left(\mathbf{V}_{h^{\prime}}^{p}, \mathbf{V}_{h^{\prime \prime}}^{p^{\prime}}\right) .
$$

Similarly,

$$
\mathbf{E}_{\mathbf{V}, \bar{\Omega}}=\bigoplus_{p, p^{\prime} \in[1, \nu]} \mathbf{E}_{\mathbf{V}, \bar{\Omega}}^{p, p^{\prime}} \quad \text { where } \mathbf{E}_{\mathbf{V}, \bar{\Omega}}^{p, p^{\prime}}=\bigoplus_{h \in \bar{\Omega}} \operatorname{Hom}\left(\mathbf{V}_{h^{\prime}}^{p}, \mathbf{V}_{h^{\prime \prime}}^{p^{\prime}}\right) .
$$

As in [L2, 10.4], the subspace $\bigoplus_{p \leq p^{\prime}} \mathbf{E}_{\mathbf{V}, \Omega}^{p, p^{\prime}}$ of $\mathbf{E}_{\mathbf{V}}$ is contained in the tangent space $T_{y}$ to the $G_{\mathbf{v}}$-orbit of $y$.

Since $\psi_{i}(y+z)=0$ for all $i$, we see from 12.8(a) that $\left\langle z, T_{y}\right\rangle=0$. Hence $\left\langle z, \bigoplus_{p \leq p^{\prime}} \mathbf{E}_{\mathbf{V}, \Omega}^{p, p^{\prime}}\right\rangle=0$. But the annihilator in $\mathbf{E}_{\mathbf{V}, \bar{\Omega}}$ of $\bigoplus_{p \leq p^{\prime}} \mathbf{E}_{\mathbf{V}, \Omega}^{p, p^{\prime}}$ under 
$\langle$,$\rangle is clearly \bigoplus_{p<p^{\prime}} \mathbf{E}_{\mathbf{v}, \bar{\Omega}}^{p, p^{\prime}}$. Hence we have $z \in \bigoplus_{p<p^{\prime}} \mathbf{E}_{\mathbf{v}, \bar{\Omega}}^{p, p^{\prime}}$. If we denote $\mathbf{V}^{(q)}=\bigoplus_{p: p>q} \mathbf{V}^{p}$, we have

$$
\begin{gathered}
\mathbf{V}=\mathbf{V}^{(0)} \supset \mathbf{V}^{(1)} \supset \cdots \supset \mathbf{V}^{(\nu)}=0 \\
z_{h}\left(\mathbf{V}_{h^{\prime}}^{(q)}\right) \subset \mathbf{V}_{h^{\prime \prime}}^{(q+1)}
\end{gathered}
$$

for all $h \in \bar{\Omega}, q \in[0, \nu-1]$ and

$$
y_{h}\left(\mathbf{V}_{h^{\prime}}^{(q)}\right) \subset \mathbf{V}_{h^{\prime \prime}}^{(q)}
$$

for all $h \in \Omega, q \in[0, \nu]$.

Now $y$ is certainly nilpotent as an element in $\mathbf{E}_{\mathbf{v}}$ (since $y \in \mathbf{E}_{\mathbf{V}, \Omega}$ and our graph is a tree); $y$ satisfies the definition of nilpotency in 1.7 with $N=|I|$. By 1.8(b) applied to $y$ and $\mathbf{V}^{p}$ we see that the filtration (d) can be refined to a flag $\phi$ in $\mathbf{V}$ that is stable under $y$.

Then $\phi$ is automatically $z$-stable. Indeed, let ${ }^{l} \mathbf{V}$ be a member of this flag. We have $\mathbf{V}^{(q)} \supset{ }^{l} \mathbf{V} \supset \mathbf{V}^{(q+1)}$ for some $q \in[0, \nu-1]$. From (e) it follows that $z_{h}\left({ }^{l} \mathbf{V}_{h^{\prime}}\right) \subset \mathbf{V}_{h^{\prime \prime}}^{(q+1)}$; hence $z_{h}\left({ }^{l} \mathbf{V}_{h^{\prime}}\right) \subset{ }^{l} \mathbf{V}_{h^{\prime \prime}}$ for all $h \in \bar{\Omega}$, which shows that $\phi$ is $z$-stable. Now $\phi$ is stable under $y$ and under $z$, and hence it is stable under $x=y+z$. Using now $1.8(\mathrm{a})$, we see that $x$ is nilpotent. The proposition is proved.

\section{EXAMPLE: GRAPHS OF AFFINE TYPE $A$}

15.1. In this section we assume that $n \geq 2, I=\mathrm{Z} / n$, and that $H$ consists of the arrows

$$
\begin{aligned}
& i \rightarrow j \quad \text { with } i, j \in I, i-j=1, \\
& i \leftarrow j \quad \text { with } i, j \in I, i-j=1 ;
\end{aligned}
$$

by definition, the involution ${ }^{-}: H \rightarrow H$ interchanges $i \rightarrow j$ and $i \leftarrow j$; if $h=(i \rightarrow j)$, we set $h^{\prime}=i, h^{\prime \prime}=j$; if $h=(i \leftarrow j)$, we set $h^{\prime}=j, h^{\prime \prime}=i$. Let $\Omega$ be the subset of $H$ consisting of the arrows (a).

Thus our graph is an affine Dynkin graph of type $A_{n}$ and $\Omega$ is an orientation of it. We shall take $\varepsilon(i \rightarrow j)=1, \varepsilon(i \leftarrow j)=-1$.

The purpose of this section is to give an explicit combinatorial parametrization for the set of irreducible components of $\Lambda_{\mathbf{V}}$ for any $\mathbf{V} \in \mathscr{V}$.

15.2. Let $k^{\prime} \leq k$ be two integers. We define $\mathbf{V}\left(k^{\prime}, k\right) \in \mathscr{V}$ to be the $\mathbf{k}$-vector space with basis $e_{r} \quad\left(r \in\left[k^{\prime}, k\right]\right), I$-graded by the requirement that $e_{r}$ has degree $i \in I$ where $r \equiv i(\bmod n)$. Let $x\left(k^{\prime}, k\right) \in \mathbf{E}_{\mathbf{V}\left(k^{\prime}, k\right), \Omega}$ be defined by $e_{r} \rightarrow e_{r-1}$ for all $r \in\left[k^{\prime}, k\right]$, where $e_{k^{\prime}-1}$ is interpreted as zero. It is clear that $\left(\mathbf{V}\left(k^{\prime}, k\right), x\left(k^{\prime}, k\right)\right)$ is an indecomposable representation of our oriented graph, with $x\left(k^{\prime}, k\right)$ nilpotent; the isomorphism class of this representation does not change when $k^{\prime}, k$ are simultaneously translated by a multiple of $n$. 
Conversely, if $(\mathbf{V}, x)$ is an indecomposable finite-dimensional representation of our oriented graph, with $x \in \mathbf{E}_{\mathbf{V}, \Omega}$ nilpotent, then it is isomorphic to a $\left(\mathbf{V}\left(k^{\prime}, k\right), x\left(k^{\prime}, k\right)\right)$ as above; moreover, $\left(k^{\prime}, k\right)$ are uniquely determined up to simultaneous translation by a multiple of $n$.

Let $Z$ be the set of all pairs $\left(k^{\prime} \leq k\right)$ of integers defined up to simultaneous translation by a multiple of $n$; let $\widetilde{Z}$ be the set of all functions $Z \rightarrow \mathbf{N}$ with finite support.

It follows that, given $\mathbf{V} \in \mathscr{V}$, the set of $G_{\mathbf{v}}$-orbits on the set of nilpotent elements in $\mathbf{E}_{\mathbf{v}, \Omega}$ is naturally indexed by the subset $\tilde{Z}_{\mathbf{v}}$ of $\tilde{Z}$ consisting of all functions $f: Z \rightarrow \mathbf{N}$ with finite support such that

$$
\sum_{k^{\prime} \leq k} f\left(k^{\prime}, k\right) \sharp\left\{r \mid k^{\prime} \leq r \leq k, r \equiv i \quad(\bmod n)\right\}=\operatorname{dim} \mathbf{V}_{i}
$$

for all $i \in I$; the sum is taken over all $k^{\prime} \leq k$ up to simultaneous translation by a multiple of $n$. This indexing is obtained by attaching to a nilpotent element $x \in \mathbf{E}_{\mathbf{v}, \Omega}$ the following function $f$ : we write $(\mathbf{V}, x)$ as a direct sum of indecomposable modules and $f\left(k^{\prime}, k\right)$ is the number of summands isomorphic to $\left(\mathbf{V}\left(k^{\prime}, k\right), x\left(k^{\prime}, k\right)\right)$. In particular,

(a) $G_{\mathbf{V}}$ has only finitely many orbits on the set of nilpotent elements in $\mathbf{E}_{\mathbf{V}, \Omega}$. We shall denote by $\mathscr{O}_{f}$ the $G_{\mathbf{v}}$ orbit corresponding to $f \in \tilde{Z}_{\mathbf{v}}$.

15.3. An element $f \in \widetilde{Z}_{\mathbf{v}}$ is said to be aperiodic if it satisfies the following condition: for any $k^{\prime} \leq k$, not all integers $f\left(k^{\prime}, k\right), f\left(k^{\prime}+1, k+1\right), \ldots$, $f\left(k^{\prime}+n-1, k+n-1\right)$ are $>0$.

15.4. For any $r \in I$, let $\operatorname{End}_{r}(\mathbf{V})$ be the vector space of all linear maps $T: \mathbf{V} \rightarrow$ $\mathbf{V}$ such that $T\left(\mathbf{V}_{i}\right) \subset \mathbf{V}_{i+r}$ for all $i \in I$.

We may identify $\mathbf{E}_{\mathbf{V}, \Omega}\left(\right.$ resp. $\mathbf{E}_{\mathbf{v}, \bar{\Omega}}$ ) with End $_{-1}(\mathbf{V})$ (resp. $\operatorname{End}_{1}(\mathbf{V})$ ). (To $x \in \mathbf{E}_{\mathbf{V}, \Omega}$ we associate the $T$ such that $T(v)=x_{i \rightarrow i-1}(v)$ for all $i$ and all $v \in \mathbf{V}_{i} ;$ to $z \in \mathbf{E}_{\mathbf{V}, \bar{\Omega}}$ we associate the $T$ such that $T(v)=z_{i+1 \leftarrow i}(v)$ for all $i$ and all $v \in \mathbf{V}_{i}$.)

The following statement is obvious.

(a) Let $x \in \mathbf{E}_{\mathbf{V}, \Omega}, z \in \mathbf{E}_{\mathbf{v}, \bar{\Omega}}$, and let $T, S$ be the corresponding elements in $\operatorname{End}_{-1}(\mathbf{V})$ and $\operatorname{End}_{1}(\mathbf{V})$. Then $x$ is nilpotent as an element of $\mathbf{E}_{\mathbf{V}, \Omega}$ if and only if $T$ is nilpotent as an endomorphism of $\mathbf{V} ; z$ is nilpotent as an element of $\mathbf{E}_{\mathbf{V}, \bar{\Omega}}$ if and only if $S$ is nilpotent as an endomorphism of $\mathbf{V}$.

The following statement is easily verified.

(b) Let $x, x^{\prime} \in \mathbf{E}_{\mathbf{v}, \Omega}$ be nilpotent elements, let $T, T^{\prime}$ be the corresponding elements of $\operatorname{End}_{-1}(\mathbf{V})$, and let $f, f^{\prime}$ be the corresponding elements of $\tilde{Z}_{\mathbf{v}}$. Assume that there exists $S \in \operatorname{End}_{1}(\mathbf{V})$ that is invertible as a linear map $\mathbf{V} \rightarrow \mathbf{V}$ such that $T S=S T^{\prime}$. Then $f\left(k^{\prime}, k\right)=f^{\prime}\left(k^{\prime}-1, k-1\right)$ for all $k^{\prime} \leq k$.

Proposition 15.5. Let $f \in \tilde{Z}_{\mathbf{v}}$. The following two conditions are equivalent.

(a) The conormal bundle of $\mathscr{O}_{f}$ consists entirely of nilpotent elements in $\mathbf{E}_{\mathbf{v}}$.

(b) $f$ is aperiodic. 
If $x \in \mathbf{E}_{\mathbf{V}, \Omega}$ and $z \in \mathbf{E}_{\mathbf{v}, \bar{\Omega}}$ then

$$
\psi_{i}(x+z)=x_{i+1 \rightarrow i} z_{i+1 \leftarrow i}-z_{i \leftarrow i-1} x_{i \rightarrow i-1} \text { for all } i \in I .
$$

Using $12.8(\mathrm{a})$, we see that condition (a) is equivalent to the following condition:

(c) Let $x \in \mathscr{O}_{f}$. Then for any $z \in \mathbf{E}_{\mathbf{v}, \bar{\Omega}}$ such that

$$
x_{i+1 \rightarrow i} z_{i+1 \leftarrow i}=z_{i \leftarrow i-1} x_{i \rightarrow i-1}
$$

for all $i \in I, x+z$ is nilpotent.

For $x$ and $z$ as in (c), the condition that $x+z$ is nilpotent is equivalent to the condition that $z$ is nilpotent (since $x$ is known to be nilpotent). Using 15.4(a), we see that (c) is equivalent to the following condition:

(d) Let $x \in \mathscr{O}_{f}$ and let $T$ be the corresponding element of $\operatorname{End}_{-1}(\mathbf{V})$. Then any $S \in \operatorname{End}_{1}(\mathbf{V})$ such that $T S=S T$ (as endomorphisms of $\mathbf{V}$ ) is nilpotent (as an endomorphism of $\mathbf{V}$ ).

Let $x, T$ be as in (d) and assume that $S \in \operatorname{End}_{1}(\mathbf{V})$ commutes with $T$ and is not nilpotent. Consider the canonical decomposition $\mathbf{V}=\mathbf{V}^{\prime} \oplus \mathbf{V}^{\prime \prime}$ such that $\mathbf{V}^{\prime}, \mathbf{V}^{\prime \prime}$ are $S$-stable, and $S$ is invertible on $\mathbf{V}^{\prime}$ and nilpotent on $\mathbf{V}^{\prime \prime}$. Then $\mathbf{V}^{\prime} \neq 0$. The previous decomposition is automatically compatible with the $I$-grading (since $S \in \operatorname{End}_{1}(\mathbf{V})$ ); moreover, $\mathbf{V}^{\prime}$ and $\mathbf{V}^{\prime \prime}$ are $T$-stable (since $T S=S T)$. Hence our decomposition is a decomposition of $(\mathbf{V}, x)$ as a direct sum of two representations of our oriented graph. Let $f^{\prime} \in \widetilde{Z}_{\mathbf{v}^{\prime}}, f^{\prime \prime} \in \widetilde{Z}_{\mathbf{v}^{\prime \prime}}$, be attached to these two representations as in 15.2. It is clear that $f=f^{\prime}+f^{\prime \prime}$. Hence to prove that $f$ is not aperiodic it is enough to show that $f^{\prime}$ is not aperiodic. Thus we can assume that $\mathbf{V}=\mathbf{V}^{\prime}$ so that $S: \mathbf{V} \rightarrow \mathbf{V}$ is invertible. Applying 15.4(b) (with $T^{\prime}=T$ ) we see that $f\left(k^{\prime}, k\right)=f\left(k^{\prime}-1, k-1\right)$ for all $k^{\prime} \leq k$. This shows that $f$ is not aperiodic. Thus we have proved that if (d) does not hold, then (b) does not hold.

We now show that if $f$ is not aperiodic, then (d) does not hold. Since $f$ is not aperiodic, we can find $k^{\prime} \leq k$ and a direct sum decomposition $\mathbf{V}=\mathbf{V}^{1} \oplus \mathbf{V}^{2} \oplus$ $\cdots \oplus \mathbf{V}^{n+1}$ of the representation $(\mathbf{V}, x)$ of our oriented graph such that the $l$ th summand is isomorphic to the representation $\left(\mathbf{V}\left(k^{\prime}+l, k+l\right), x\left(k^{\prime}+l, k+l\right)\right)$ (see 15.2) for $l=1,2, \ldots, n$. It is then clear that there exists a linear map $S \in \operatorname{End}_{1}(\mathbf{V})$ such that $S=0$ on $\mathbf{V}^{n+1}$ and $S$ is an isomorphism of the vector space $\mathbf{V}^{1} \oplus \mathbf{V}^{2} \oplus \cdots \oplus \mathbf{V}^{n}$ onto itself, commuting with $T$. This shows that (d) does not hold. The proposition is proved.

Corollary 15.6. For any $f \in Z_{\mathbf{v}}$, let $\mathscr{C}_{f}$ be the conormal bundle of $\mathscr{O}_{f}$; let $\overline{\mathscr{C}}_{f}$ be the closure of $\mathscr{C}_{f}$.

Then $f \rightarrow \overline{\mathscr{C}}_{f}$ is a 1-1 correspondence between the set of aperiodic elements in $Z_{\mathbf{v}}$ and the set of irreducible components of $\Lambda_{\mathbf{v}}$.

Let $X$ be an irreducible component of $\Lambda_{\mathrm{V}}$. The image of $X$ under the canonical projection $\mathbf{E}_{\mathbf{V}} \rightarrow \mathbf{E}_{\mathbf{V}, \Omega}$ (with kernel $\mathbf{E}_{\mathbf{V}, \bar{\Omega}}$ ) is denoted $X^{\prime}$. It is 
contained in the set of nilpotent elements of $\mathbf{E}_{\mathbf{v}, \Omega}$ since $X$ consists of nilpotent elements. Moreover, it is $G_{\mathbf{v}^{-}}$-stable. Hence it is the union of finitely many $G_{\mathbf{v}^{-}}$ orbits. Let $\mathscr{O}_{f_{1}}, \ldots, \mathscr{O}_{f_{r}}$ be these orbits. By $12.8(\mathrm{a}), X$ is contained in the union $\mathscr{C}_{f_{1}} \cup \cdots \cup \mathscr{C}_{f_{r}}$. Each of these conormal bundles is irreducible of the same dimension as $X$. It follows that $X$ must be equal to the closure of one of these conormal bundles; hence $X=\overline{\mathscr{C}}_{f}$ for some $f$ that, by 15.5 , is necessarily aperiodic. Conversely, if $f \in Z_{\mathbf{V}}$ is aperiodic, then any $x \in \mathscr{C}_{f}$ is nilpotent, by 15.5 , and satisfies $\psi_{i}(x)=0$ for all $i$, by $12.8(\mathrm{a})$; thus, $\mathscr{C}_{f}$ is contained in $\Lambda_{\mathrm{V}}$, and hence its closure is contained in $\Lambda_{\mathrm{V}}$. Being irreducible of the correct dimension, it is an irreducible component of $\Lambda_{\mathbf{V}}$. This completes the proof.

\section{GRAPHS WITH A CYCLIC GROUP ACTION}

16.1. In this section we assume that we are given a finite cyclic group $C$ and an action of $C$ on our graph such that the following property is satisfied. If $c \in C$ fixes an edge, then it fixes both ends of that edge; if an edge and its image under $c \in C$ have the same end points then that edge is fixed by $c$.

This induces actions of $C$ on $I$ and $H$ which are compatible with the maps 1.1(a),(b),(c); moreover, $h, \bar{h}$ are never in the same $C$-orbit on $H$.

Let $P$ be the set of orbits of $C$ on $I$. For $p \in P$ let $d_{p}$ be the number of elements in $p$. Given two distinct orbits $p, q$ in $P$ we denote by $n_{p q}$ the number of elements $h \in H$ such that $h^{\prime} \in p$ and $h^{\prime \prime} \in q$. Clearly, $n_{p q}=n_{q p}$ is divisible by both $d_{p}$ and $d_{q}$. Hence the matrix

(a) $\left(a_{p q}\right)_{p, q \in P}$ given by $a_{p p}=2$ and $a_{p q}=-d_{p}^{-1} n_{p q}($ for $p \neq q)$ is a symmetrizable generalized Cartan matrix.

16.2. Note that any nonsymmetric Cartan matrix of affine type can be obtained by the procedure of 16.1 starting from an extended Dynkin graph of type $A, D$ or $E$ and a suitable cyclic group acting on it. Consider the following extended Dynkin graphs with a faithful action of $C$ of the order indicated by a left superscript:

$$
\begin{gathered}
{ }^{2} \widetilde{A}_{2 l-1}(l \geq 2),{ }^{2} \widetilde{D}_{l+1}^{1}(l \geq 3),{ }^{2} \widetilde{D}_{l+2}^{2}(l \geq 2),{ }^{2} \widetilde{D}_{2 l}^{\geq 3}(l \geq 3), \\
{ }^{2} \widetilde{E}_{6},{ }^{2} \widetilde{E}_{7},{ }^{3} \widetilde{D}_{4},{ }^{3} \widetilde{E}_{6},{ }^{4} \widetilde{D}_{2 l+2}(l \geq 1) .
\end{gathered}
$$

(The right superscript $1,2, \geq 3$ indicates the number of nontrivial orbits of $C$ on the set of vertices.)

The corresponding Cartan matrices are (with the notations in $[\mathrm{K}, \mathrm{p} .44,45]$ ) respectively:

$$
C_{l}^{(1)}, B_{l}^{(1)}, D_{l+1}^{(2)}, A_{2 l-1}^{(2)}, F_{4}^{(1)}, E_{6}^{(2)}, G_{2}^{(1)}, D_{4}^{(3)}, A_{2 l}^{(2)} .
$$

16.3. We want to sketch the way in which the results of this paper should be extended to the case of the quantized enveloping algebra $\mathrm{U}^{-}$corresponding to the Cartan matrix 16.2(a). 
Let $\Omega$ be an orientation of our graph that is compatible with the $C$-action. (Such $\Omega$ always exists.)

Let $\mathscr{V}(C)$ be the category of finite-dimensional $I$-graded $\mathbf{k}$-vector spaces $\mathbf{V}$ with an action of $C$ that have the following properties: if $c \in C$ and $i \in I$ then $c \mathbf{V}_{i} \subset \mathbf{V}_{c(i)}$; if in addition, $c(i)=i$, then $c: \mathbf{V}_{i} \rightarrow \mathbf{V}_{i}$ is the identity.

Let $\mathbf{V} \in \mathscr{V}(C)$ be such that $\mathbf{V} \in \mathscr{V}_{\nu}$, when the $C$-action is forgotten. Note that we have a natural action of $C$ on $\mathbf{E}_{\mathbf{v}}$ preserves the subspace $\mathbf{E}_{\mathbf{v}, \Omega}$.

Let $S_{\nu, C}$ be the set of all pairs $(\mathbf{p}, \mathbf{a})$ where $\mathbf{p}=\left(p_{1}, p_{2}, \ldots, p_{m}\right)$ is a sequence of elements of $P$ and $\mathbf{a}=\left(a_{1}, a_{2}, \ldots, a_{m}\right)$ is a sequence of integers $\geq 0$ such that $\sum_{l: i \in p_{l}} a_{l}=\nu(i)$ for all $i \in I$.

Let $(\mathbf{p}, \mathbf{a}) \in S_{\nu, C}$. A $C$-flag of type $(\mathbf{p}, \mathbf{a})$ in $\mathbf{V}$ is, by definition, a sequence $\phi=\left(\mathbf{V}=\mathbf{V}^{0} \supset \mathbf{V}^{1} \supset \cdots \supset \mathbf{V}^{m}=0\right)$ of $I$-graded $C$-stable subspaces of $\mathbf{V}$ such that, for any $l=1,2, \ldots, m$, the graded vector space $\mathbf{V}^{l-1} / \mathbf{V}^{l}$ is zero in degrees $i \notin p_{l}$ and has dimension $a_{l}$ in degrees $i \in p_{l}$.

As in 1.4, we say that $\phi$ is $x$-stable (where $x \in \mathbf{E}_{\mathbf{v}}$ ) if $\mathbf{V}^{l}$ is $x$-stable for all $h \in H$ and all $l=0,1, \ldots, m$. Let $\mathscr{F}_{\mathbf{p}, \mathbf{a}}$ be the variety of all $C$-flags of type $(\mathbf{p}, \mathbf{a})$ in $\mathbf{V}$.

Let $\widetilde{\mathscr{F}}_{\mathbf{p}, \mathbf{a}}$ be the variety of all pairs $(x, \phi)$ such that $x \in \mathbf{E}_{\mathbf{V}, \Omega}$ and $\phi \in \mathscr{F}_{\mathbf{p}, \mathbf{a}}$ is $x$-stable.

We denote by $\pi_{\mathbf{p}, \mathbf{a}}: \widetilde{\mathscr{F}}_{\mathbf{p}, \mathbf{a}} \rightarrow \mathbf{E}_{\mathbf{v}, \Omega}$ the first projection.

We set $L_{\mathbf{p}, \mathbf{a} ; \Omega}=\left(\pi_{\mathbf{p}, \mathbf{a}}\right)_{!}(1) \in \mathscr{D}\left(\mathbf{E}_{\mathbf{v}, \Omega}\right)$. This is a semisimple complex which is $C$-equivariant in a suitable sense. It can be decomposed in a direct sum of summands $L[d]$ where the $L$ are $C$-equivariant perverse sheaves that are simple (as equivariant perverse sheaves, but not necessarily as perverse sheaves without $C$-action). The set of isomorphism classes of the various $L$ that appear in this way is denoted $\mathscr{P}_{\mathbf{V}, \Omega, C}$. From this point the development should continue as in the case of trivial $C$, and it should lead to a canonical basis of $\mathbf{U}^{-}$indexed by the disjoint union of the sets $\mathscr{P}_{\mathbf{V}, \Omega, C}$ (with $\mathbf{V}$ running over a set of representatives for the isomorphism classes in $\mathscr{V}^{C}$ ).

\section{REFERENCES}

[BBD] A. A. Beilinson, J. Bernstein, and P. Deligne, Faisceaux pervers, Astérisque 100 (1982).

[D] P. Deligne, La conjecture de Weil, II, Inst. Hautes Études Publ. Math. 52 (1980), 137-252.

[DR] V. Dlab and C. M. Ringel, Indecomposable representations of graphs and algebras, Mem. Amer. Math. Soc., No. 173, Amer. Math. Soc., Providence, RI, 1976.

[K] V. Kac, Infinite dimensional Lie algebras, Birkhäuser, Boston, 1983.

[KS] M. Kashiwara and P. Schapira, Microlocal study of sheaves, Astérisque 128 (1985).

[K] P. B. Kronheimer, The construction of ALE spaces as hyper-Kähler quotients, J. Differential Geom. 29 (1989), 665-683.

[L1] G. Lusztig, Character sheaves, I, Adv. in Math. 56 (1985), 193-237.

[L2] Canonical bases arising from quantized enveloping algebras, J. Amer. Math. Soc. 3 (1990), 447-498.

[L3] _ Canonical bases arising from quantized enveloping algebras, II, Progr. Theor. Phys. 102 (1990). 
[M] R. MacPherson, Chern classes for singular varieties, Ann. of Math. (2) 100 (1974), 423-432.

[R] C. M. Ringel, Hall algebras and quantum groups, Invent. Math. (1990).

[S] A. Schofield, Notes on constructing Lie algebras from finite-dimensional algebras, preprint.

Department of Mathematics, Massachusetts Institute of Technology, Cambridge, MASSACHUSETTS 02139 